\title{
Nanotheranostics
}

2019; 3(1): 1-40. doi: 10.7150/ntno.27877

Review

\section{Theragnostic potentials of core/shell mesoporous silica nanostructures}

\author{
Aswathy Ravindran Girija ${ }^{{ }^{凶 *}}$, Sivakumar Balasubramanian ${ }^{2 *}$ \\ 1. Future Industries Institute, University of South Australia Mawson Lakes Campus, Mawson Lakes 5095, SA, Australia. \\ 2. School of Engineering, University of South Australia Mawson Lakes Campus, Mawson Lakes 5095, SA, Australia. \\ *Both authors equally contributed \\ $\square$ Corresponding author: Aswathy Ravindran Girija, Research Fellow, Future Industries Institute, University of South Australia. Mawson Lakes Campus, \\ Adelaide, SA, 5095, Australia. Email: aswathy.ravindrangirija@unisa.edu.au \\ (1) Ivyspring International Publisher. This is an open access article distributed under the terms of the Creative Commons Attribution (CC BY-NC) license \\ (https://creativecommons.org/licenses/by-nc/4.0/). See http://ivyspring.com/terms for full terms and conditions.
}

Received: 2018.06.14; Accepted: 2018.09.22; Published: 2019.01.01

\begin{abstract}
Theragnostics is considered as an emerging treatment strategy that integrates therapeutics and diagnostics thus allowing delivery of therapeutics and simultaneous monitoring of the progression of treatment. Among the different types of inorganic nanomaterials that are being used for nanomedicine, core shell mesoporous silica nanoparticles have emerged as promising multifunctional nanoplatform for theragnostic application. Research in the design of core/shell mesoporous silica nanoparticles is steadily diversifying owing to the various interesting properties of these nanomaterials that are advantageous for advanced biomedical applications. Core/shell mesoporous silica nanoparticles, have garnered substantial attention in recent years because of their exceptional properties including large surface area, low density, ease of functionalization, high loading capacity of drugs, control of the morphology, particle size, tunable hollow interior space and mesoporous shell and possibility of incorporating multifunctional interior core material. In the past decade researcher's demonstrated tremendous development in design of functionalized core/shell mesoporous silica nanoparticles with different inorganic functional nanomaterial incorporated into mesoporous nanosystem for simultaneous therapeutic and diagnostic (theragnostic) applications in cancer. In this review, we recapitulate the progress in commonly used synthetic strategies and theragnostic applications of core/shell mesoporous silica nanoparticles with special emphasis on therapeutic and diagnostic modalities. Finally, we discuss the challenges and some perspectives on the future research and development of theragnostic core/shell mesoporous silica nanoparticles.
\end{abstract}

Key words: Theragnostic, Core/shell, Mesoporous silica nanoparticles, Multifunctional, Cancer, Therapeutic, Diagnostic

\section{Introduction}

Novel nanoscale materials with advanced functionalities are being developed for biomedical applications. The development of multifunctional mesoporous nanomaterials for a wide variety of scientific applications signifies their importance in various research fields, especially in the biomedical field that is heading towards the design and development of personalised medicine. The remarkable advances in the design and development of mesoporous silica nanoparticles and interaction of these nanomaterials with biological system are one of the prominent topics in material science and biological science research. In the biomedical application, mesoporous silica nanoparticles are being used for controlled and targeted drug delivery owing to its inherent mesoporous structure. Core/shell mesoporous silica nanoparticles (C/S-MSNs) have garnered immense attention in biomedical application 
of nanomaterials owing excellent features including enhanced specific surface area, huge void space to accommodate guest molecule, mesoporous channels on outer shell, reduced density and superior biocompatibility. The attractiveness of these nanostructures is that their formation, size, shape, porosity, pore volume, pore size (textural properties) thickness of mesoporous shell can be precisely tuned owing to the control in their chemistry. In addition, the effective surface modifications impart these nanostructures supplementary role of gate-keeping to prevent premature release of drugs, active targeting, and diagnostic functionalities [1-3]. C/S-MSNs projects its biomedical application as a nano-reserve for the storage of drug in their void or hollow core, controlled (smart/stimuli responsive) and sustained release of encapsulated or adsorbed drug, targeted delivery of drugs with functionalized ligands thus minimizing unfavourable side effects, and ultimately the development of theragnostic nanostructures that aids simultaneous therapeutic and diagnostic functions by utilizing the benefits of mesoporous core shell nanostructures. Theragnostic nanomaterials are those materials that reduce the gap between therapeutic efficiency and diagnostic potential thus coupling aforementioned different strategies to a single unit. To provide an overview of versatile core shell mesoporous nanostructures as theragnostic agents in cancer nanotechnology, we aim to discuss major synthetic strategies of C/S-MSNs and recent improvements in the theragnostic (therapeutic and diagnostic) applications for cancer. Firstly, we discuss significant properties and different methodology for the synthesis of core shell mesoporous silica nanostructures. In the second section, we focus on various therapeutic strategies (targeted chemotherapy, magnetic hyperthermia, photodynamic therapy, gene therapy, immunotherapy, etc.) and diagnostic modalities (optical imaging, magnetic resonance imaging, nuclear imaging, etc.) emerged from the core part of the nanocarrier. Finally, we discuss the major challenges and future perspectives of C/S-MSNs in biomedical regime.

\section{Synthetic approaches of C/S-MSNs}

C/S-MSNs are a category of mesoporous silica nanoparticles that comprise of a core and a shell. The core and shell can be made either from different materials or same materials with different structures. The core can be either hollow or nanoparticle encapsulated core with mesoporous shell. Different types of C/S-MSNs are demonstrated schematically in figure 1. It is possible to have a mesoporous shell with a hollow core, small sphere inside (a rattle-like or yolk-shell structure), aggregated core spheres, with multiple shells (with desirable pore structure). The significance of C/S-MSNs is the synergistic effect offered by the combination of desired properties of core materials, structure and biocompatibility for biomedical applications. There are several techniques reported for C/S-MSNs to improve the loading capacity of guest molecule by increasing their pore volume. When compared with conventional uniform mesoporous silica nanoparticles, the hollow C/S-MSNs offers void interior for the diffusion of chemotherapeutic agents through ordered mesoporous nanochannels from the outer surface that contribute extra mesoporous surface for the adsorption of drug molecules. The formation of mesoporous shells and hollow void interior together has been reported in several studies. Hollow mesoporous silica nanoparticles are generally synthesised by employing sacrificial templates as core or by the selective etching of the solid silica present in the interior [4]. The most commonly reported method for the synthesis of hollow C/S-MSNs is "templating method" [5]. The templating method depends on the use of soft/hard templates as substrate and coating of the shell with appropriate structure/composition onto the surface of the template used in the synthesis. The template is finally removed and hollow C/S-MSNs can be obtained. (Figure 2) Based on the type of template used for the formation of void interior, the synthesis of hollow or C/S-MSNs are broadly categorized into soft templating and hard templating approaches.

\section{Soft templating approaches}

In soft templating approach, the hollow structure and mesopores are simultaneously developed through self- assembly of the precursor molecules and organic surfactant templates. The templates that are generally used as core are mostly droplets of "soft" precursor molecules, surfactants, or organic additives that are removed leading to the generation of hollow C/S-MSNs. The mesoporous nanochannels are developed by the surfactant templates/ micelles, which are eliminated in subsequent steps. The soft templates used in this approach include micelles, micro emulsion droplets or vesicles. Core templates are removed by extraction or calcination resulting in the hollow nanostructure formation. In "self-templating" the precursor molecule droplets itself function as core template, and those molecules are used for generating hollow structures, and are used up in the formation of the mesostructured shells; thereby eliminating the core template removal by procedures such as calcination or extraction [6]. 

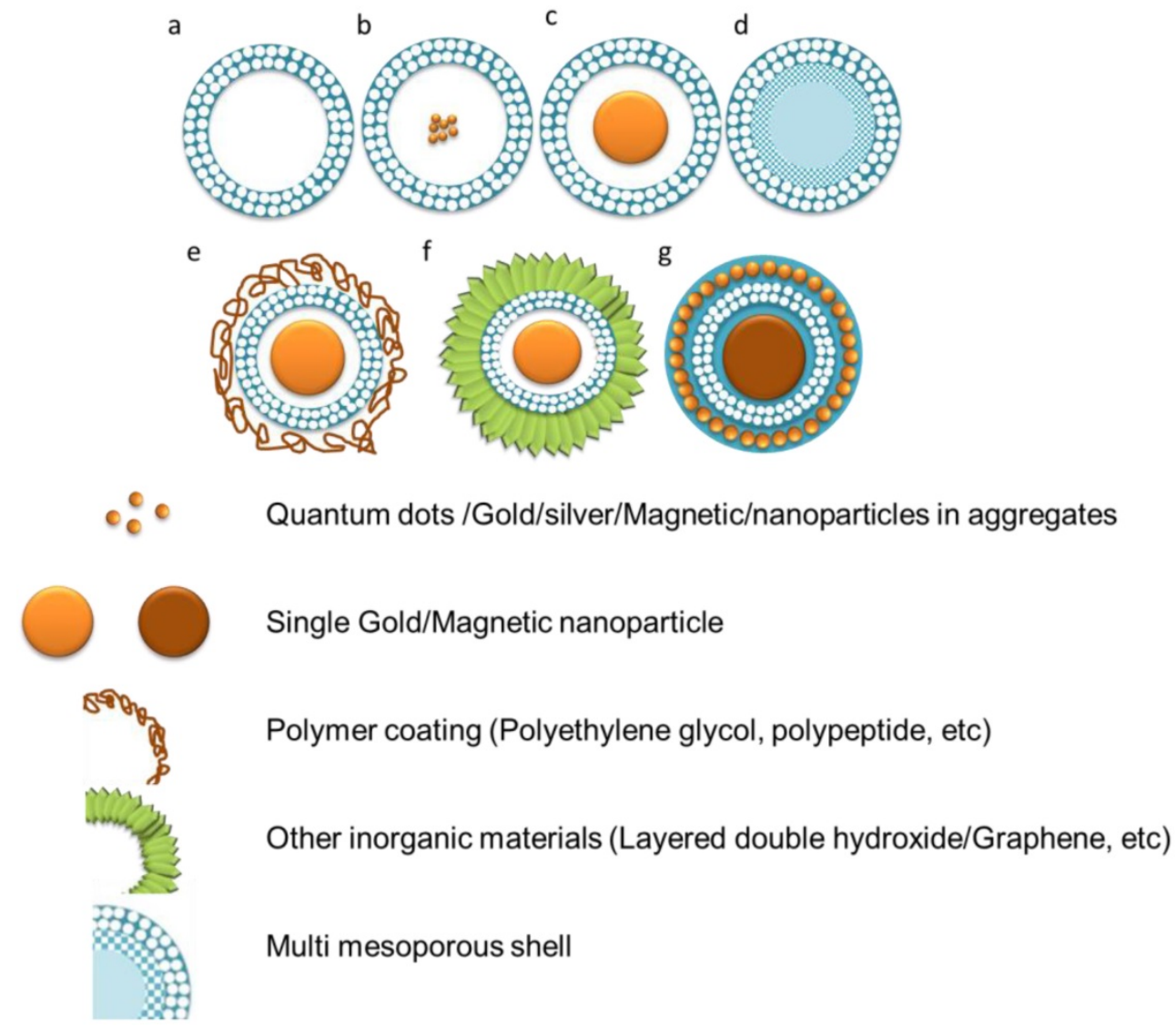

Figure 1: Schematic representation of various types of C/SMSNs. a) Hollow core shell mesoporous silica nanoparticles, b) Multiple cores single shell c) Single core mesoporous shell, d) Multi mesoporous shells, e) Single core mesoporous shell with polymer coating, d) Complex core-shell structures, where single core mesoporous shell attached to other inorganic nanostructures with different functionalities and g) Complex core-shell structures by the incorporation of smaller spheres into the shell or with multiple shells.

\section{Single micelle templating}

Very small hollow C/S-MSNs in the range of 20-40 nm are formed based on single micelle templating where micelle templates are generated and silica is deposited on the templates. During the development of nanoparticles, in soft templating approach, the cross linking of micelle/silica does not arise. In this approach, the curvature of micelles can be controlled by varying the polymer combination, interactions within polymer chains or by adding adequate amount of micelle swelling agent [7]. Mandal et al, used P127 (EO $\left.\mathrm{EO}_{106} \mathrm{PO}_{70} \mathrm{EO}_{106}\right)$ block copolymer templated synthesis where the lower framework precursor to surface ratio assisted the formation of hollow nanoparticles of different size that were templated by single micelles [8]. Bao et al developed hollow C/S-MSNs of $\sim 500 \mathrm{~nm}$ with hollow cavity by micelle template method. They used cetyl trimethyl ammonium bromide (CTAB) micelle as cavity and mesoporous templates that made them convenient and easy for the preparation and removal at ambient condition [9]. The researchers also demonstrated the influence of dissolving methods of $\mathrm{CTAB}$, ethanol-to-water volume ratio on the morphology of developed hollow C/S-MSNs. The cationic surfactant formed micelles in the ethanol-water systems where rich-ethanol phase was inside micelles and a rich-water phase was outside micelles due to the differences in dielectric constant. The silica source tetraethoxysilane (TEOS) was diffused into the ethanol rich hydrophobic interior phase of CTAB micelles to form "oil-in-water" emulsion, where the "oil" was TEOS. The "oil-in-water" emulsion served as temporary soft template to design the structure of hollow C/S-MSNs. In the presence of ammonia as catalyst, TEOS present inside the micelles hydrolysed and condensed to create silica seeds and the growth was from outside to inside along micelle template. (Figure 3)

\section{Vesicle templating}

Owing to the chemistry of surfactants (cationic and anionic co-surfactants), a range of aggregate with different shapes and morphologies including micelles, rod/cylindrical shaped micelles, vesicles, lamellar phases and liquid-crystal phases, are generated that function as templates for hollow C/S-MSNs [10]. Vesicles are self-organized template from surfactants 
with delicate bilayer shells. The addition of inorganic components such as source of silica leads to solid vesicle structures and finally the template is removed via calcination or extraction. Yeh et al employed neutral tri-block copolymer as ternary surfactant to introduce mesopores to the shell of hollow silica nanoparticles. In their study, aqueous cationic surfactants (CTAB and anionic SDS) were used to develop dual surfactant micelles and tri-block copolymer Pluronic P123 $\left(\mathrm{EO}_{20} \mathrm{PO}_{70} \mathrm{EO}_{20}\right)$ was introduced that functioned as surfactant on the micelle exterior surface and directed the development of mesoporous silica shell. The template was eliminated by calcination that led to the generation of hollow C/S-MSNs of 100 to $500 \mathrm{~nm}$ with hollow shell and pore size of $5 \mathrm{~nm}$. Stable vesicle templates were developed from cationic fluorinated surfactant (FC4) with CTAB or F127. Hollow silica nanoparticles with mesoporous walls were developed from co-assembly of silica with cationic fluorinated surfactants. Thus mesoporous organosilica hollow structures with a controllable wall thickness and multishelled mesoporous structure was developed through composite vesicle templates that were formed via the interaction of cationic fluorinated surfactants, CTAB and negatively charged silica species [11].

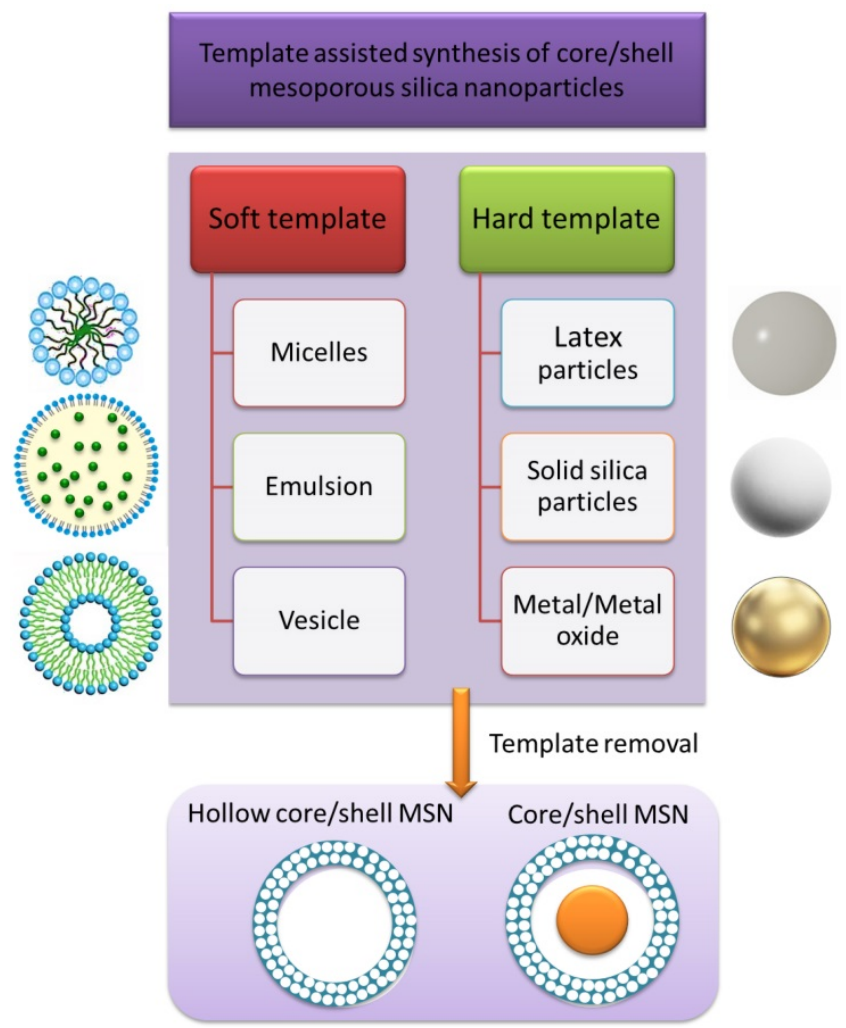

Figure 2: Template assisted synthesis of C/SMSN and representative materials as template.

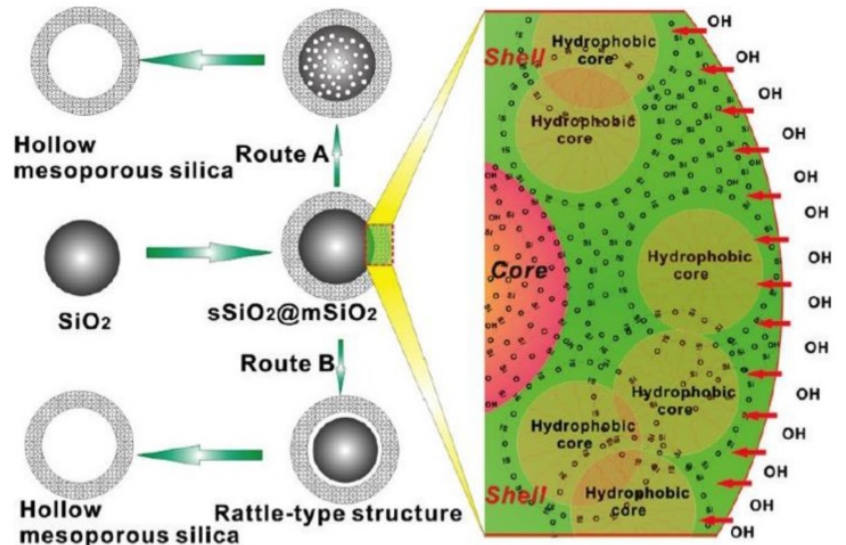

Figure 3: Schematic of the formation of hollow/rattle-type mesoporous silica spheres (left) and the microscopic structure schematics (right) based on selective-etching procedure in $\mathrm{Na}_{2} \mathrm{CO}_{3}$ solution (route $\mathrm{A}$ ) and in ammonia solution under hydrothermal treatment (route B) [22].

\section{Microemulsion templating}

Oil-in-water $(\mathrm{O} / \mathrm{W})$ and water-in-oil (W/O) microemulsion is another soft template synthetic approach, which is characterized by the dispersion of solvent in dispersed phase in a continuous phase with opposite hydrophilicity in the presence of surfactant to form homogeneous droplets as soft template. Hollow silica nanoparticles have been synthesized from stable $\mathrm{O} / \mathrm{W}$ microemulsion developed from the mixture of water, oil, surfactant and aqueous alkaline solution. Li et al developed hollow C/S-MSNs, where $\mathrm{N}, \mathrm{N}$-dimethylformamide (DMF) has been used as the dispersed phase of the O/W synthesis and the interior of hollow C/S-MSNs with radially orientated mesopore structures was adjusted by varying DMF/water ratio [12]. This method is often used for encapsulating other nanoparticles (quantumdots, $\mathrm{Au}$ nanoparticles, $\left.\mathrm{Fe}_{3} \mathrm{O}_{4}\right)$ in the hollow interior nanoparticles resulting in C/S-MSNs [13].

In the soft-templating approach the presence of mesostructures with low rigidity leads to formation of particles with non-uniform size distribution, mixed mesostructures and morphology. The precise size control of droplet leading to the formation of polydispersed hollow C/S-MSNs is considered as one of the major drawbacks of soft template assisted synthesis. Monodispersed discrete nanoparticles with good colloidal stability are essential for biomedical applications. The lack of specific control over reaction and presence of aggregation of hollow C/S-MSNs often results in complications that may adversely affect the suspensibility of these nanoparticles in solution for biomedical application. Therefore, hard-core templating approaches are employed to overcome the difficulties associated with soft templating approaches. 


\section{Hard templating approach}

High quality monodisperse hollow C/S-MSNs are being synthesized via hard templating approach. Monodispersed hollow C/S-MSNs with controllable shell thickness, hollow core size, and mesoporosity of the shell is attained via hard-core templating strategy. The porous shell are formed by the self-assembly between the precursor and mesoporogen on the surface of hard-core and aggregation of nanoparticles is often prevented which is significant in biomedical application [14]. The hollow cavity inside mesoporous shell is formed due to the compositional and structural difference in the hard-core and shell where the core material is etched. The method depends on hard-core materials (polystyrene sphere, dense silica, iron oxide nanoparticles) and mode of removal of core material. Different types of etchants including sodium hydroxide $(\mathrm{NaOH})$, sodium carbonate $\left(\mathrm{Na}_{2} \mathrm{CO}_{3}\right)$, hydrochloric acid $(\mathrm{HCl})$, hydrofluoric acid $(\mathrm{HF})$, and hot water have been reported for removal of core material [15-18].

\section{Polymer latex beads}

Among various colloidal hard template, polymer latex beads [polystyrene (PS), polymethylmethacrylate (PMMA)] are widely used to synthesize hollow C/S-MSNs owing to their uniform size and ease of removal of organic matter by calcination [19]. In hard template approach appropriate surface functionalization for interfacial recognition of silicates are to be performed prior to silicification on organic template. Tan et al [20] demonstrated PS as hard-core template for the synthesis of hollow silica nanoparticles. PS hard-core template of size that varies from $350 \mathrm{~nm}$ to $450 \mathrm{~nm}$ was developed from emulsion polymerization method. The nanosilica, originated from TEOS under moist alkaline condition at ambient state was coated over the surface of PS templates. PS cores were removed by calcination at $500{ }^{\circ} \mathrm{C}$. Further stabilization of PS surface with polyvinylpyrrolidone (PVP) and modification of PS beads with functional molecules accelerated silica deposition. This could be due to the positively charged PS spheres, facile environment for electrostatic attraction, or the introduction of catalyst to initiate sol-gel process of TEOS [21]. In addition to PS, there are several reports on poly(acrylic acid) (PAA), polymethylmethacrylate (PMMA) and its copolymeric nanospheres as hard templates for the synthesis of hollow C/S-MSNs [22].

\section{Silica as hard template}

Silica nanoparticles have been widely reported as hard-core template for the synthesis of hollow C/S-MSNs owing to its uniformity, tunable size and other unique features [22]. Chen et al demonstrated the fabrication of silica "nanorattles" using organic-inorganic hybrid silica spheres as hard template [16]. The selective etching with HF resulted in the formation of a small silica core inside hollow cavity with a mesoporous shell. The size, mesoporous shell thickness, and core diameter of the monodisperse nanorattles was controlled precisely. This method was showcased as a promising method for the synthesis of rattle-type functional nanomaterials. Shi and co-workers reported preparation of hollow/rattle-type mesoporous silica nanostructure through selective etching strategy based on structural difference. Solid silica core was prepared and mesoporous silica shell ( $\left.\mathrm{sSiO}_{2} @ \mathrm{mSiO}_{2}\right)$ was deposited on colloidal silica surface by co-condensation of n-octadecyltrimethoxysilane $\left(\mathrm{C}_{18} \mathrm{TMS}\right)$ and TEOS [17]. The condensation degree of silicate in meosopore shell layer was higher when compared to solid silica core that was developed by self-assembly of $\mathrm{C}_{18} \mathrm{TMS}$ and TEOS, and by Stöber method. The researchers employed two strategies to create hollow or rattle-type mesoporous silica shell. In the first approach, prior to the removal of surfactants, $\mathrm{sSiO}_{2} @ \mathrm{mSiO}_{2}$ was treated with $\mathrm{Na}_{2} \mathrm{CO}_{3}$ solution. $\mathrm{Na}_{2} \mathrm{CO}_{3}$ treatment aided the formation of small pores inside silica core that resulted in the development of pore due to the collapse of small pores, finally leading to the formation of hollow or rattle type mesoporous silica nanostructure. In the second approach, hydrothermal treatment in ammonia solution was employed. Treatment of $\mathrm{sSiO}_{2} @ \mathrm{mSiO}_{2}$ in higher concentration of ammonia solution for a longer period eventually resulted in the formation of hollow interiors.

\section{Other hard templates}

Apart from polymeric templates and metallic oxide templates, several inorganic templates are also reported for the synthesis of hollow C/S-MSNs. Advantages of these templates include the synthesis is free from organic solvents and the surface properties can be maintained as such before silica coating. There are reports on $\mathrm{CaCO}_{3}$ nanoparticles as templates for the synthesis of hollow C/S-MSNs. Shape controlled hollow mesoporous silica nanoparticles have been demonstrated by using cubic, rough-surfaced spherical and rod-like $\mathrm{CaCO}_{3}$ as inorganic particle template approach which involve sol-gel silica coating over surfaces of the template and the template was further removed by acid-dissolution [23]. Wang et al reported shape-controlled synthesis of hollow silica colloids by employing hematite as hard template [24]. In their study, hematite colloidal particles with different shapes (pseudocubes, 
ellipsoids, capsules, and peanuts) was prepared and coated with silica to develop hematite core-silica shell structure. (Figure 4) Finally hematite cores were removed in $\mathrm{HCl}$ solution leading to the formation of

hollow silica colloids of different shapes.

a
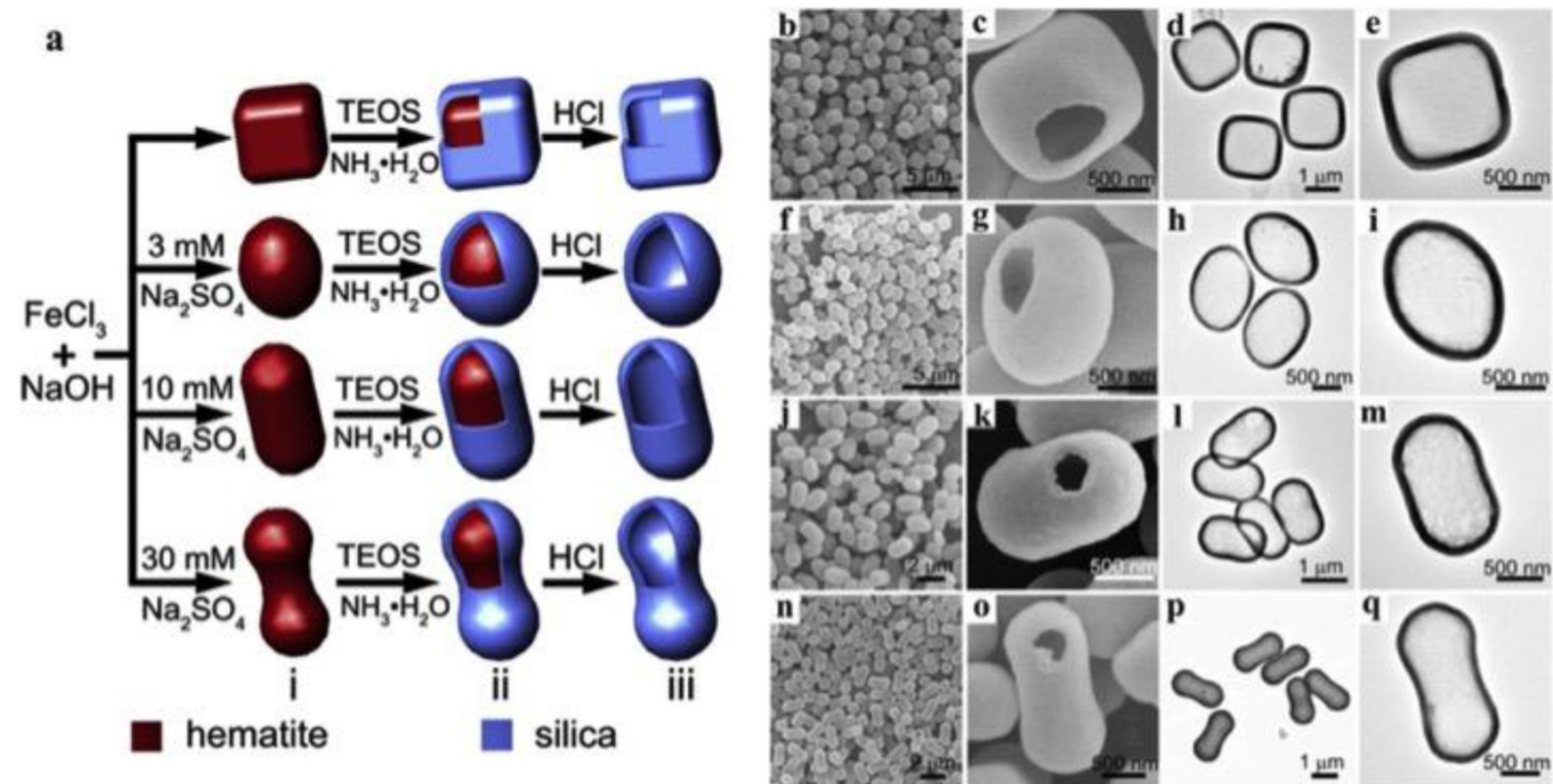

Figure 4: (a) Schematic of the shape-controlled synthesis of hollow silica colloids and FESEM and TEM images of hollow silica colloids with different shapes: (b-e) pseudocubes, $(f-i)$ ellipsoids, $(j-m)$ capsules, and $(n-q)$ peanuts [30].
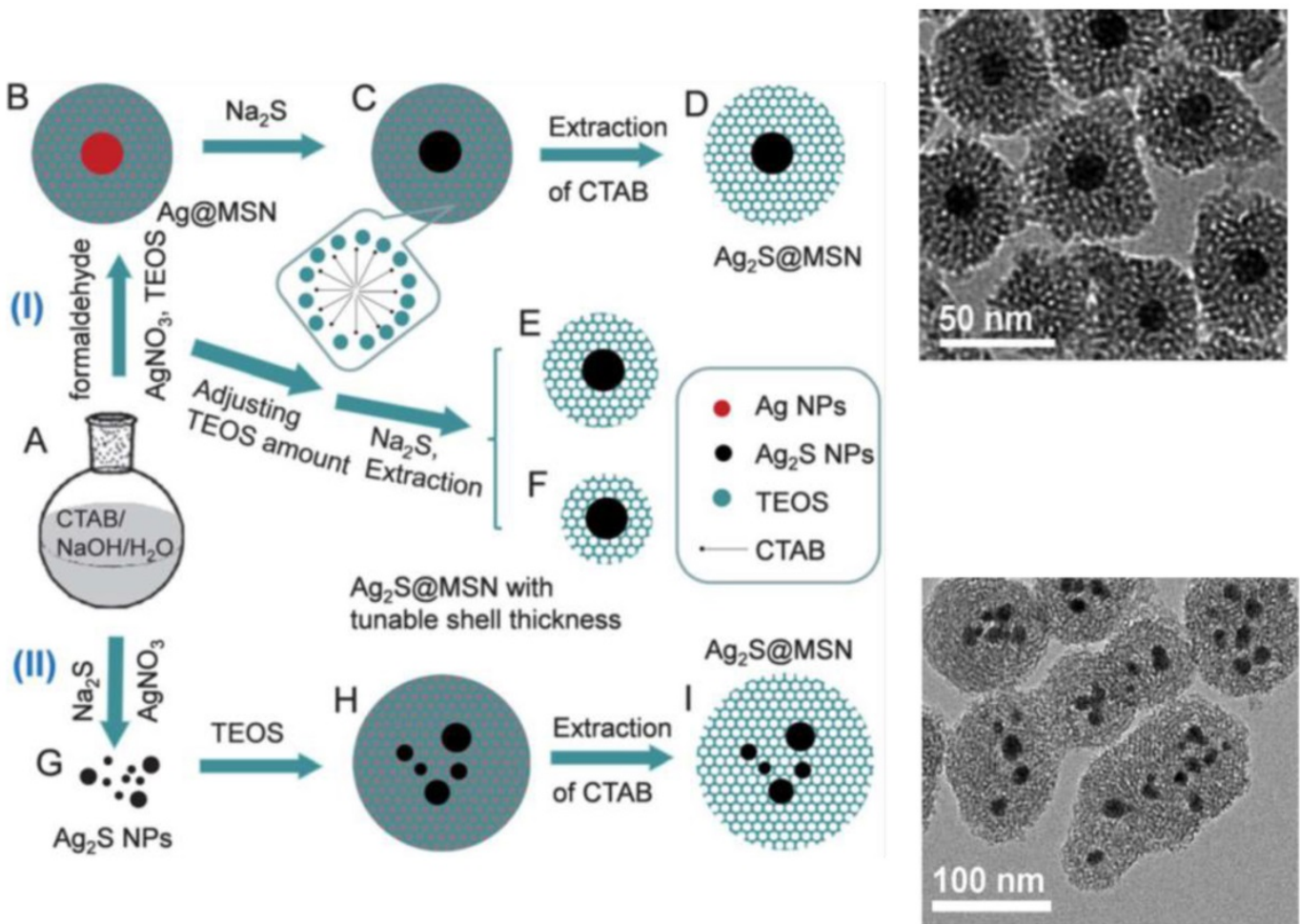

Figure 5: The schematic of the formation of the $\mathrm{Ag}_{2} \mathrm{~S} @ M S N$ nanocomposites. Route (I) depicts the formation of core-shell $\mathrm{Ag} 2 \mathrm{~S} @ \mathrm{MSN}$ nanospheres with a single $\mathrm{Ag}_{2} \mathrm{~S}$ nanoparticle core in one mesoporous silica shell. Route (II) depicts the formation of core-shell $\mathrm{Ag}_{2} \mathrm{~S} @ M S N$ nanospheres with several $\mathrm{Ag} 2 \mathrm{~S}$ nanoparticles in one mesoporous silica shell and their respective TEM images [44]. 


\section{Metal or metal/oxide/sulphide nanoparticles as templates}

There are several reports on the application of other hard templates including metal, metal oxides or semiconductor nanomaterials and subsequent formation of mesoporous silica layer to develop metal or metal oxide nanoparticles@MSNs as tailored nanoparticles for multifunctional application. With a good control on reaction conditions and by the choice of surfactants and polymers, these core materials can be encapsulated or embedded in mesoporous silica shell. The advantages of silica shell over metal or metal nanoparticles include the biocompatibility of mesoporous silica shell, ease of surface functionalization for biomedical applications, and non-interference of the inherent properties of metal or metal oxide embedded in core [localized surface plasmon resonance (SPR) of noble metal nanoparticles, magnetic properties of magnetic nanoparticles, optical properties of semiconductor quantumdots/ upconversion nanoparticles]. The metal or metal oxide nanoparticles@MSN combines advantages of both (metallic/metal oxide core and mesoporous shell) materials, to overcome any limitation of bare core nanomaterials with specific properties.

Several studies have been reported on the synthesis and application of ferromagnetic metallic nanoparticles as core with mesoporous silica shell [25, 26]. Magnetic iron oxides including magnetite, hematite and maghemite are widely used owing to their inherent superparamagnetic properties [27]. However bare iron oxide nanoparticles exhibits high agglomeration in aqueous phase and high chemical reactivity towards air/aqueous environment that may affect their magnetism and dispersibility. Mesoporous silica shell on these iron oxide nanoparticles stabilizes the core by enhancing their dispersibility in aqueous and biological buffer solutions and also offer the possibility for surface functionalization for biomedical applications. Kim et al reported fabrication of monodispersed magnetite $\left(\mathrm{Fe}_{3} \mathrm{O}_{4}\right)$ nanocrystals and both magnetite nanocrystals and quantum dots (CdSe/ZnS) into uniform mesoporous silica nanospheres [28]. In the study CTAB was employed as a secondary surfactant and as organic template for synthesis of mesoporous silica nanospheres. CTAB-stabilized magnetite nanocrystals functioned as nuclei for the growth of spherical mesoporous silica nanoparticles. The sol-gel reaction of TEOS, CTAB and oleic acid stabilized magnetite nanocrystals and subsequent removal of organic templates generated magnetite nanocrystals and CdSe/ZnS quantum dots embedded mesoporous silica spheres. The same researchers further modified the surface of monodisperse single $\mathrm{Fe}_{3} \mathrm{O}_{4}$ nanocrystal@mesoporous silica with polyethylene glycol (PEG) for their biomedical application [15]. PEG offered excellent biocompatibility by inhibiting nonspecific protein adsorption to nanoparticles. PEGylated $\mathrm{Fe}_{3} \mathrm{O}_{4} @$ mesoporus silica was further used as $\mathrm{T}_{2}$-weighted contrast agent for in vivo MRI.

Owing to the inert nature, biocompatibility, ease of surface modification and possessing the inherent properties including SPR, Au nanoparticles is widely used in the development of biosensor and drug delivery vehicles. Although there are reports on capping molecules for preventing the aggregation of $\mathrm{Au}$ nanoparticles, after certain period of time, particles tend to aggregate owing to chemical degradation or due to chemical reactivity depending on the ionic strength, $\mathrm{pH}$, and temperature [29]. Mesoporous silica shell prevents aggregation of $\mathrm{Au}$ nanoparticles and bestows nanoparticle with additional functionalities [30]. The possibility of tuning the thickness of mesoporous silica layer affects SPR effect of Au nanoparticles [31]. Lee et al reported the synthesis of Au cores and hollow silica shells with vacant internal space by adopting selective etching strategy of the $\mathrm{Au}$ cores from $\mathrm{Au} @ \mathrm{SiO}_{2}$ core/shell nanoparticles by the treatment of potassium cyanide $(\mathrm{KCN})$ [32]. The developed nanoparticles (AuNR@SiO ${ }_{2}$ ) could overcome the major drawback of $\mathrm{Au}$ nanorod (AuNR), specially reduced surface area for loading drug and aggregation inside the cells. CTAB shaped a bilayer around AuNRs and functioned as an organic template for the development of mesoporous silica layer.

As mentioned previously, nanomaterials that serve as core in the development of metal/metal oxide/sulphide@ $\mathrm{msiO}_{2}$ nanoparticles often possess definite role in the development of theragnostic application. Han et al demonstrated fabrication of $\mathrm{Ag}_{2} \mathrm{~S}$ nanoparticles as core and deposition of mesoporous $\mathrm{Si}$ as shell [33]. The developed $\mathrm{Ag}_{2} \mathrm{~S} @ \mathrm{MSN}$ mesoporous silica nanospheres exhibited excellent near-infrared (NIR) photoluminescent properties that can be exploited for NIR bio-imaging. The formation of $\mathrm{Ag}_{2} \mathrm{~S} @ \mathrm{MSN}$ was demonstrated under two approaches. (Figure 5) The first approach defined the formation of single Ag nanoparticle core in one mesoporous silica shell. Ag@MSN nanoparticles were synthesized that reacted with sodium sulfide that converted rapidly to $\mathrm{Ag}_{2} \mathrm{~S}$ in presence of oxygen resulting in the formation of uniform core-shell $\mathrm{Ag}_{2} \mathrm{~S} @ \mathrm{MSN}$ nanospheres. The thickness of mesoporous silica shell over the $\mathrm{Ag}_{2} \mathrm{~S}$ core could be tuned by adjusting the amount of TEOS. In the second approach, numerous small $\mathrm{Ag}_{2} \mathrm{~S}$ 
nanoparticles were covered by mesoporous silica shell to form core shell nanoparticles. The developed core/shell nanoparticles could potentially function as excellent nanovector for delivery of drugs and bio imaging.

\section{Other approaches}

Apart from the discussed soft and hard template assisted synthesis of hollow C/S-MSNs, aerosol assisted approach for direct synthesis of dry hollow mesoporous nanoparticles has been reported. Aerosols generated from processes including thermal spraying, evaporation or salt decomposition may serve as templates for synthesis of hollow mesoporous nanoparticles. Aerosol fabrication approach offers an easy and scalable method for developing hollow mesoporous nanoparticles and metal oxide hollow spheres with uniform size and tunable textural properties. $\mathrm{Lu}$ et al reported aerosol-based procedure for synthesis of solid and mesoporous silica spheres with stable mesoporous structures of hexagonal and cubic topology and with layered structures [34]. The method was based on evaporation-induced interfacial self-assembly (EISA) on spherical aerosol droplets. Electrospray method is used for the fabrication of nanospheres, hollow nanomaterials [organic, inorganic, hybrid (inorganic-organic) hollow nanostructures] and nanofibers. Suhendi et al used electrospray method for synthesis of hollow nanoparticles by the assembly of colloidal nanoparticles [35]. The researchers fabricated hollow colloidosomes by eletrospraying silica and PS nanospheres on a collection electrode. The self-assembly of colloidal nanoparticles within charged droplets allowed the formation of porous and hollow particles. PS cores were finally removed at high temperature to form hollow structured nanoparticles.

\section{Theragnostic mesoporous silica nanoparticles for biomedical applications}

Theragnostic is the generic name given to the class of nanostructures which can offer benefits of therapeutic and diagnostic property simultaneously and bestow the nanostructure with multifunctional properties. Both therapeutic and diagnostic entities are well organised into a single compartment which can be the future of personalized medicine. Integration of diagnostic functionalities to therapeutic functionality could eventually result in the choice of therapy and treatment strategy based on the type of cancer along with detection and monitoring the progress of treatment. Hollow mesoporous core/shell based nanoparticles owing to its bio-compatibility and ease of bio-functionalization is a promising treatment and diagnostic approach for several diseases, especially for cancer. The inherent properties of hollow nanostructures including low density, very high surface area, large pore volume, tunable pore size, large interior void space along with optical, magnetic properties or photoacoustic offered by core material, make hollow nanostructures as ideal candidates for theragnostic applications [36]. The hollow space inside the mesoporous shell serves as micro-/nanoreservoir of drug for drug delivery and the incorporation of diagnostic components for bio imaging applications. A thorough understanding of interaction of physiochemical properties of hollow mesoporous nanostructures with biological system is highly recommended for their application in biology as there can be strong influence of morphology, size, chemistry, textural parameters on biological system. The study on bio-nano interaction can enable the development of nanoparticles with specific functionalities for biomedical applications. Apart from physiochemical properties, shape features including the aspect ratio and morphology is often given importance as it may affect the performance. For example aspect ratio can lead to several orientations in the bionano interface and it can influence circulation time, internalization rate, tumor therapy efficacy etc [5, 37]. MSNs with different morphologies including hollow/rattle type, multishell type, metal/metaloxide@mSiO2 owing to their distinctive spatial architectures have garnered significant consideration that could influence their biomedical applications. Advances in the synthetic parameters of hollow/rattle MSNs have led to design of nanoparticles with large void space to encapsulate more amount of drug for drug delivery purposes [38].

Nanoparticles become significant for drug delivery when the right delivery platform is chosen. Enhanced drug loading capacity with controlled and sustained release of drugs from the targeted nanocarrier is an ideal alternate to conventional therapeutic approaches. The remarkable combination of treatment and detection of cancer using mesoporous nanostructures has garnered considerable attention in recent years. The well-established siloxane chemistry of mesoporous silica nanoparticles and distinct physicochemical characteristics such as high surface to volume ratio, tunable pore size, and accessibility to incorporate anticancer drugs, dyes, contrast agents, favours this significant class of nanomaterial to elevate themselves to versatile theragnostic class of nanomaterials. These properties along with the flexibility of surface modification and excellent biocompatibility make mesoporous core/shell silica-based nanoparticles as ideal candidates for therapeutic and diagnostic 
applications. During last decade, with the intervention of nanotechnology, significant research was being focused both in the nanotherapeutics and nanodiagnostic, as different entities. However the coupling of therapeutic and diagnostic modalities and its immense potential could revolutionize current scenario of deadly cancer [39]. Multifunctional mesoporous silica nanoparticles decorated with appropriate targeting moieties can be an incredible tool for sensitive tracking of cancer coupled with fine straightforward therapy, which can kill cancer cells and sparing healthy normal neighbouring cells. Application of different types of therapeutic and diagnostic approaches based on C/S-MSNs is discussed. (Figure 6)

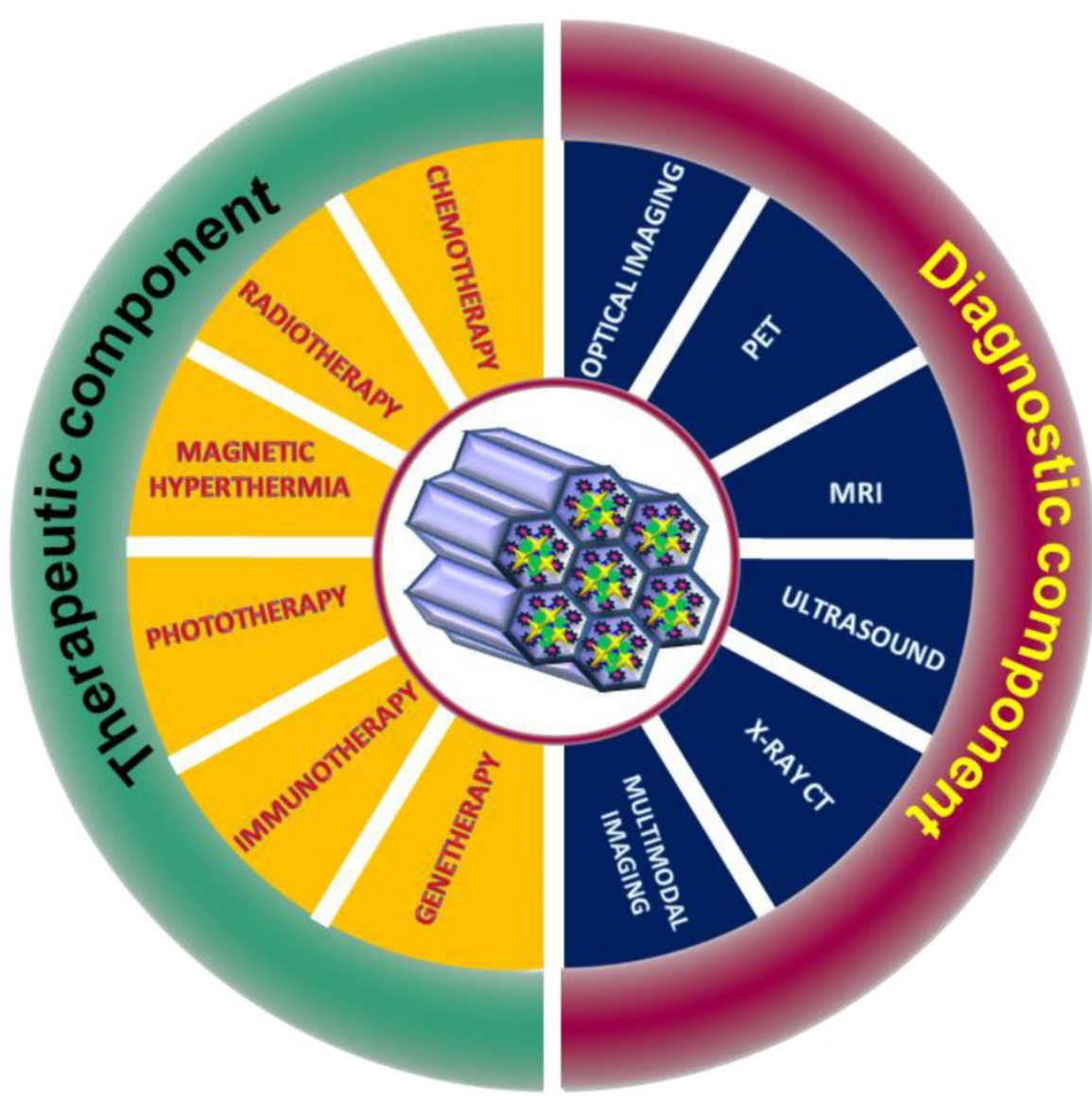

Figure 6: Theragnostic applications of C/SMSNs

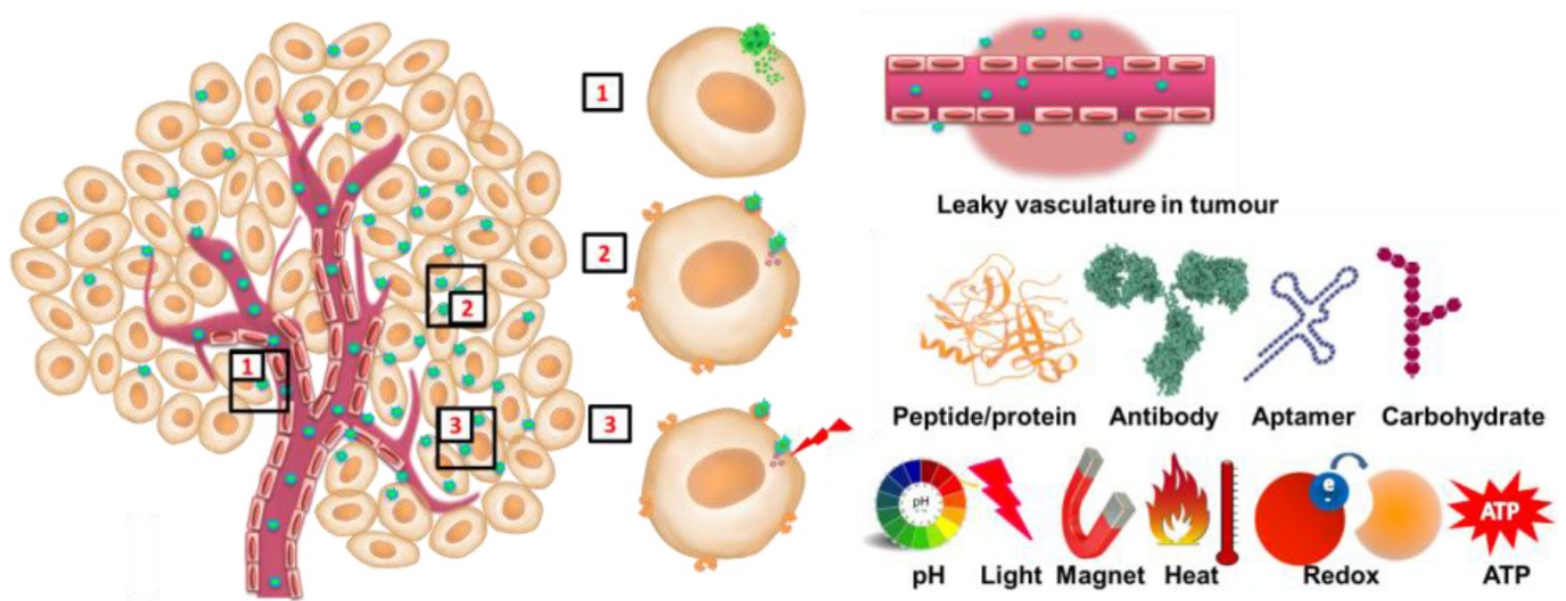

Figure 7: Schematic of 1) passive targeting, 2) active targeting and 3) stimuli responsive release of cargo. 


\section{Therapeutic applications}

In this review we discuss some major therapeutic strategies including chemotherapy, radiotherapy, magnetic hyperthermia, phototherapy, gene therapy and immunotherapy that have been reported with C/S-MSNs.

\section{Chemotherapy}

The use of C/S-MSNs for applications in drug delivery grants a wide range of advantages [40]. It includes a) the ease of functionalization with surface and conjugation chemistry permitting the attachment of various targeting moieties and incorporation of cytotoxic drugs, b) advantage of enhanced permeation and retention effect (EPR), c) targeted delivery of drug/cargo to tumour tissue thus sparing healthy neighbouring cells, d) enhanced penetration into cells depending on core materials for therapy and deep tissue imaging, e) ultra large pores for high drug payload loadings, f) controlled release kinetics depending on core material/ interaction with drug and/or capping mechanism, g) integration of a stimuli-responsive molecular regulator into nanocarrier that prevent premature release of drug before reaching target site, etc. Researchers are currently exploring these advantages of C/S-MSNs to enhance the potential of targeted drug delivery as a therapeutic choice.

Nanoparticles including C/S-MSNs take advantage from surface properties that enable them to accumulate in tumour tissue owing to EPR effect to attain 'passive targeting' and with decorated targeting ligands for 'active targeting' that enhances drug concentration and reduces nonspecific biodistribution of drugs [41]. (Figure 7). Conjugation of affinity ligands to mesoporous nanoparticles is an excellent strategy to target cancer specific cells and sparing normal cells. Targeted nanocarrier precisely recognizes and binds to cancer by ligand-receptor interaction and delivers cargo (anticancer drugs) to targeted cancer cells actively and effectively. Commonly used targeting ligands to mesoporous silica nanoparticles include several small molecules (folic acid), antibodies, peptides and proteins, aptamers, saccharides [42-49]. Although targeted nanoparticles deliver drug to targeted cells, another important property to be considered is the controlled release. There has been extensive research in design and development of stimuli responsive release of drugs from nanostructures for cancer therapy. A major challenge for the design and practical application of any nanoparticle for cancer therapy is the controlled delivery. Stimuli responsive nanocarriers are significant owing to their capability for controlled and sustained delivery of cargo (drug) to target cancerous site. These stimuli responsive nanocarriers are designed to release their cargo upon an endogenous (enzymes, $\mathrm{pH}$ and other biomolecules) or exogenous (temperature, ultrasound, external magnetic field, irradiation of near infrared radiation, etc) stimuli. (Figure 7)

Among various stimuli-responsive drug delivery systems, $\mathrm{pH}$ - responsive drug delivery system is most studied owing to the variations in $\mathrm{pH}$ exhibited by the human body. When compared with normal healthy cells, cancer cells are more acidic. Hypoxia-induced up regulation of glycolysis and subsequent accumulation of lactate in tumour cells, high interstitial pressure in the internal environment of tumours, leaky and disorganized vasculature accounts for acidic $\mathrm{pH}$ in tumour tissues $(\mathrm{pH}<7)$ [50]. Consequently, pH-responsive mesoporous drug delivery systems have been chosen as feasible approach to accomplish site-specific controlled drug release system. Several techniques are reported for the design of $\mathrm{pH}$-responsive drug delivery and few of them are with polyelectrolytes gatekeepers, $\mathrm{pH}$-sensitive linkers, supramolecular nanovalves, acid-decomposable inorganic gatekeepers, etc. Polyelectrolytes are polymers composed of recurring groups that carry electrolyte units, which are either adsorbed or covalently linked to the surface of mesoporous silica nanoparticles that can transform under different $\mathrm{pH}$ values. Polyelectrolytes remain wrapped around nanoparticles and prevent the opening of pores and release of drugs. Some of the reported polyelectrolytes for $\mathrm{pH}$ responsive drug release includes poly (allylamine hydrochloride), sodium poly(styrene sulfonata), poly[2(diethylamino)ethyl methacrylate], poly (glutamic acid), poly(4-vinyl pyridine) chitosan and poly(acrylic acid) [51-53]. At acidic $\mathrm{pH}$, polyelectrolytes around mesoporous nanoparticles may swell resulting in the release of cargo from pores [54]. The supramolecular nanovalve developed based on supramolecular chemistry serve as gatekeeper for controlled release of cargo. It is based on stalk molecule that is covalently linked to surface of silica and a movable cyclic molecule enclosing the stalk through non-covalent interactions. Meng et al developed an acid responsive $\beta$-cyclodextrin ( $\beta$-CD) nanovalves on mesoporous silica nanoparticles for controlled release of drugs [55]. N-methylbenzimidazole was immobilized as stalks that bind to $\beta$-CD rings at $\mathrm{pH} 7.4$ thus entrapping cargo in nanopores. The detachment of $\beta$-CD caps at acidifying endosomal compartment triggered the release of doxorubicin loaded in nanoparticles. Feng and coworkers reported $\mathrm{pH}$-responsive nanogate system by capping gold 
nanoparticle by acid-labile acetal linker onto mesoporous silica nanoparticles [56]. The pores were blocked by gold nanoparticles and prohibited the release of drugs at neutral $\mathrm{pH}$. At acidic $\mathrm{pH}$, owing to the hydrolysis of grafted acetal group, gold cap was separated resulting in the release of cargo from nanoparticles. In some studies, acidic-decomposable inorganic materials are used as gatekeepers for $\mathrm{pH}$ responsive drug release. Chen and co-workers reported a $\mathrm{pH}$-responsive controlled drug release nanosystem with acid-degradable layered double hydroxides (LDHs) as inorganic nanovalves [57]. $\mathrm{LDH}$ nanosheets were electrostatically adsorbed on the surface of mesoporous silica nanoparticles. The cargo, $\left(\mathrm{Ru}(\mathrm{bpy})_{3} \mathrm{Cl}_{2}\right)$ was loaded and encapsulated in a neutral environment and acidic $\mathrm{pH}$ triggered the dissolution of LDH coatings resulting in the release of cargo from mesoporous silica nanoparticles. In another study, $\mathrm{ZnO}$ QDs were used to block nanopores of mesoporous silica nanoparticles [58]. Acid-degradable QDs ( $\mathrm{ZnO})$ were dissolved inside endosomes resulting in the release of drugs from mesoporous silica nanoparticles into cytosol.

A moderate increase in temperature is generally observed in tumours, during infections and inflammation. Tumour cells are very active owing to uncontrolled cell division resulting in a rise in temperature compared to normal cells. The difference in temperature is used as a stimulus to trigger release of drugs from nanoparticles. Temperature sensitive polymers, magnetic nanoparticles or near infra-red light (NIR) are often employed to trigger the release of drug upon increase in temperature. Poly (N-isopropylacrylamide) (PNIPAAM) and its derivatives are most commonly employed temperature-sensitive polymers for temperature controlled drug release [59]. These polymers remain hydrated and swollen form that generates a diffusion bottleneck preventing the drug release below a lower critical solution temperature (LCST). When the temperature exceeds LCST, reversible phase transition is observed where water is excluded from these polymers resulting in a shrunken hydrophobic state and subsequent opening of pores and release of drug from nanoparticle [60]. Lopez and co-workers modified inner surface of mesoporous silica nanoparticles through atom transfer radical polymerization (ATRP) and demonstrated the release of drugs at higher temperature from PNIPAMfunctionalized mesoporous silica nanoparticles [61]. Release of drug was inhibited at lower temperature signifying temperature responsive drug release from mesoporous silica nanoparticles [61, 62]. Duguet and co-workers developed core-shell nanoparticles of maghemite core and a mesoporous silica shell as 'on demand' heat-triggered drug release system [63]. Doxorubicin was loaded within mesoporous cavities and 1-tetradecanol (TD), a phase-change molecule with a melting temperature $\left(T_{\mathrm{m}}\right)$ of $39{ }^{\circ} \mathrm{C}$ was presented as gatekeepers to control drug release pattern. A "zero premature release" of drug was observed under physiological conditions $\left(37^{\circ} \mathrm{C}\right)$, and a sustainable release of drug was observed above $T_{\mathrm{m}}$ of TD $\left(40^{\circ} \mathrm{C}\right)$. The study also confirmed the prospect to deliver smaller drug cargos by pulsatile release mode via multiple heating on/off cycles, owing to reversible phase change of phase-change molecules. In vitro studies demonstrated that continuous cell apoptosis was observed at temperature above $T_{\mathrm{m}}$ of $\mathrm{TD}$, owing to heat-triggered release of DOX. A thermally degradable core-shell $\mathrm{Fe}_{3} \mathrm{O}_{4} @ \mathrm{SiO}_{2}$ mesoporous silica nanoparticle in which drug release was based on magnetic stimuli (exposure to external magnetic field) was developed by Saint-Cricq et al [64]. Researchers used azo-functionalised poly-(ethylene glycol) (PEG) as a coating and to seal mesopores of silica nanoparticles. Polymer was thermo-responsive permitting the release of drug with increase in temperature breaks the covalent bonds, thus triggering the release of drug. The researchers advocate that thermo-responsive polymer coated core shell mesoporous silica nanoparticles are excellent candidates for delivering therapeutics in cancer therapy and it was the first report on a polymer that establishes the breaking of covalent bond (azo bond) at a physiologically relevant temperature. In addition to PNIPAAM, several biocompatible polymers including sulfobetaine copolymers, poly(ethyleneoxideb- $N$-vinylcaprolactam) and copolymer-lipid bilayers have been reported to exhibit temperature responsive release of drugs [65, 66].

Among various controlled release system, light triggered drug release has gained considerable attention owing to its non-invasiveness, remote responsiveness and controllable operation. Light with specific wavelength is being exposed to nanoparticles loaded with drugs, to control the release of cargo. Tanaka and co-workers reported first light-responsive controlled release system with UV-light sensitive coumarin as capping moieties on the surface of mesoporous silica nanoparticles where they demonstrated controlled drug release via light-controlled and reversible intermolecular dimerization of coumarin derivatives linked to the pores of mesoporous silica nanoparticles [67]. Cyclobutane rings of coumarin dimers were cleaved by UV light at $250 \mathrm{~nm}$ resulting in the release of drug. Several photo responsive linkers including, thymine, azobenzene, o-nitrobenzyl ester, graphene oxide and 
aluminium phthalocyaninedisulfonate were effectively used as photo- controlled capping and releasing reagents that respond to light of different wavelengths subsequently releasing the stored cargo. Researchers are focusing to gain the benefits of near-infrared (NIR) and far-infrared- (F-NIR) responsive controlled release systems owing to its deep tissue penetration properties. Tang et al reported graphene oxide (GO) as photochemical responsive linker and demonstrated controllable drug release where NIR light was used as external stimuli to elicit the release of doxorubicin (Dox) [68]. When GO wrapping was tumbled upon laser irradiation, "off-on" photo-responsive drug delivery system was activated, thus stimulating chemotherapy. (Figure 8) Shi and co-workers developed upconversion nanoparticle coated with mesoporous silica that was modified to control drug release upon near-infrared (NIR) irradiation by adjusting time duration and intensity of radiation. They coated $\mathrm{NaYF}_{4}: \mathrm{Tm}$, $\mathrm{Yb} @ N a Y F_{4}$ with azobenzene group-modified

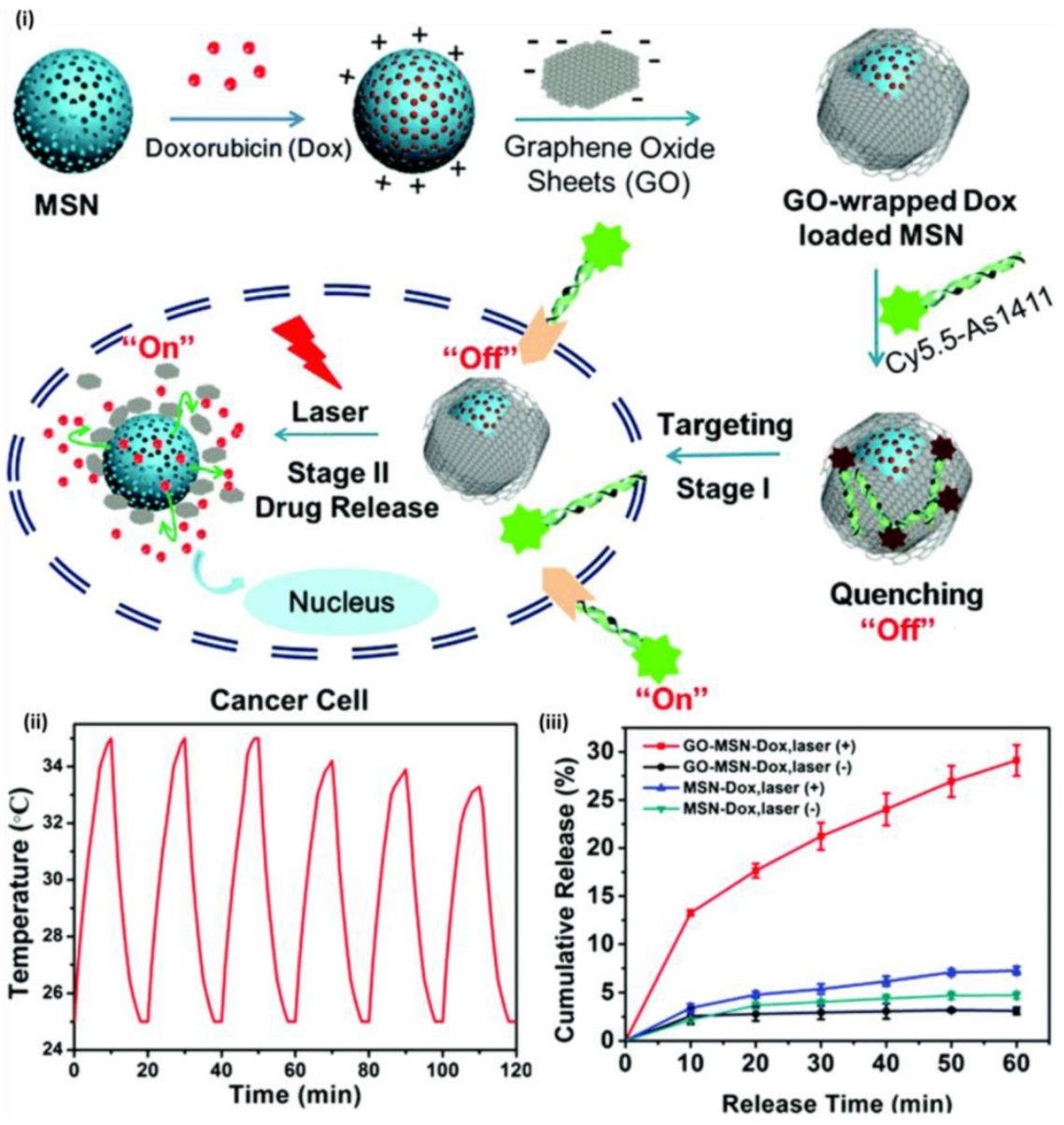

Figure 8: (i) Schematic of GO-wrapped Dox-loaded MSN-NH 2 bound with Cy5.5-labeled AS1411 aptamer and the corresponding NIR light-controlled intracellular drug release. The two "off-on" switches of MSN-Dox@GO-Apt are controlled by aptamer targeting and light triggering, respectively (ii) A temperature plot of MSN-Dox@GO solution irradiated by an $808 \mathrm{~nm}$ laser $\left(0.25 \mathrm{~W} / \mathrm{cm}^{2}\right)$ for six on-off cycles (on: $10 \mathrm{~min}$, off: $10 \mathrm{~min}$ ) and Dox release profiles of MSN-Dox@GO and MSN-Dox with and without NIR laser irradiation [81]. mesoporous silica nanoparticles and light at $980 \mathrm{~nm}$ was used to control the release of loaded drug [69].

Adenosine 5'-triphosphate (ATP) is abundantly present in the cytosol is being used as a stimulus to trigger drug release from nanoparticles. Zhu et al reported controlled drug release system based on mesoporous silica nanoparticles gated with aptamer-modified $\mathrm{Au}$ nanoparticles [70]. In their study, mesoporous silica nanoparticles were functionalized with adenosine- $\mathrm{COOH}$, which was a derivative of ATP target to generate MSA. Au nanoparticles were functionalized with ATP aptamer to form AuNPs-aptamer. When the AuNPs- aptamer was mixed with MSA, AuNPs blocked the pores of MSA due to the binding of adenosine with ATP aptamer. Au nanoparticles were uncapped and in presence of ATP molecule due to competitive displacement reaction and the entrapped fluorescein isothiocyanate (FITC) dye was released signifying ATP triggered release of cargo. Recently Lai et al developed a polypeptide enveloped mesoporoussilica-coated multicolour upconversion nanoparticle (UCNP@MSN) that functioned as ATP responsive drug delivery for real-time monitoring of drug release studies and long-term tracking [71]. The core/shell UCNP@MSN drug delivery system comprises of two components: (i) a UCNP core when excited at $980 \mathrm{~nm}$, emitted distinct emission peaks in UV to NIR region, and (ii) a mesoporous silica shell-based "biogate" for ATP-responsive stimuli for drug release. ATP-responsive drug release involved zincdipicolylamine analogue (TDPA-Zn2p) immobilized on the external surface of nanoparticle, which functioned as binding sites for capping ligands, and anticancer drug was loaded in interior mesopores. Luminescence resonance energy transfer (LRET) was observed from incorporated UCNPs owing to the entrapped drugs that resulted in quenching of UCNP emission in UV-visible region of spectrum while holding strong NIR emission. The addition of ATP to the system resulted in competitive displacement of polypeptide (surface bound) by 
ATP owing to its affinity to TDPA-Zn2p, leading to the discharge of the drugs and interdict of LRET. ATP-induced ratiometric changes in LRET permitted real time monitoring of drug release and demonstrated its potential in cancer as well as stem cells.

\section{Radiotherapy}

Extensive research in cancer science has resulted in deep understanding of molecular mechanism of the progression of cancer and has contributed several therapeutic strategies. Among several therapeutic strategies for cancer treatment, radiotherapy is one of the most efficient therapies against solid tumours. An ideal radiation therapy modality is expected to offer cellular damage abundantly to cancer cells, sparing healthy neighbouring tissue. However a major criterion for the subsequent recurrence of tumour is the resistance to therapeutic strategy. Hence radiotherapy in addition to chemotherapy after surgical removal of tumour is generally adapted for cancer treatment in many cases. Although radiotherapy is efficient, owing to its own inherent properties, radiotherapy function unevenly in different tissues thereby damaging both tumour cells and surrounding healthy cells. The application of modern technology including image-guided radiation therapy (IGRT), 3D-CRT (three-dimensional conformal radiotherapy) and IMRT (intensity modulated radiation therapy) exhibited certain side effects in neighbouring healthy cells [72].

To overcome immediate adverse effects of radiotherapy including cytotoxic effects and off targets effects of radiosensitizers research focusing on the development of nanomaterials for selective application of ionizing radiation has been developing. The research mainly focus on design and development of versatile nanoparticles with the capability of encapsulation, slow and sustained release of drugs, minimizing the possibility of immune-clearance and targeting tumour tissue. Radiation sensitization is a procedure of augmenting predisposition of tumour tissues to get destroyed by the exposure to radiation. Radiosensitizers are agents that boost the effects of radiation therapy. Radiotherapeutic effects offered by gold-based nanoparticles are gaining special attention over last few years. Metal nanoparticles with high atomic number $(\mathrm{Z})$ owing to their excellent radiosensitization effect are gaining considerable attention in recent times. Their strong photoelectric absorbance, generation of short-range secondary electrons, rapid DNA damage destroys tumour cells during radiotherapy. Also nanosized particles exhibit enhanced tumour accumulation owing to enhanced permeability and retention (EPR) effect of leaky tumour vasculature. Thus integration of radiosensititaion effect owing to enhanced accumulation of targeted nanoparticles in tumour can deliver ideal dosage of radiation to tumour region, sparing healthy tissue. Special consideration while designing nanoformulation with radioisotope is provided, that the content should not be released prematurely or degraded before reaching target tumour site, thus confirming the desired high radiation dosage for damaging the cancer cells.

As silica is categorized under non-metal radiosensitizers, it has been employed as an efficient nanocarrier or as a potential coating material in nanoformulation covering heavy metals for radiosensitization. Soares et al. developed silica nanoparticles as nanocarriers of ${ }^{159} \mathrm{Gd}-\mathrm{DTPA}-\mathrm{BMA}$ (gadolinium diethylenetriaminepentaacetic acid bismethylamide) [73]. Neutron irradiation can turn gadolinium present in Gd-DTPA-BMA, to become radioactive where ${ }^{158} \mathrm{Gd}$ isotope absorbs a neutron and converts to ${ }^{159} \mathrm{Gd}$ radioisotope through neutron/gamma nuclear reaction. The nanocarriers with ${ }^{159} \mathrm{Gd}$ radioisotope could specifically distribute radiation doses to tumours and avoiding off target tissues from getting large radiation dose. Gadolinium complex integrated into mesoporous silica nanoparticles was significantly improved, reaching more than $80 \%$ upon prolonged incubation. The researchers advocates that enhanced incorporation rates accounts for the interactions between Gd-DTPA-BMA and silanol groups in the porous and surfaces of mesoporous silica matrices. Mesoporous silica nanoparticles exhibited exciting release profile, where very low quantities of gadolinium complexes were released in a simulated body fluid. The release pattern was $1.24 \%, 1.23 \%, 1.21 \%$ and $1.29 \%$ for time intervals of $24,48,72$ and $160 \mathrm{~h}$ respectively signifying Gd complex binds strongly with silica nanoparticles. Mesoporous silica nanoparticles did not undergo substantial degradation even after incubating for 160 $\mathrm{h}$, demonstrating in vitro stability of nanoparticles. Thus silica nanoparticles incorporated with ${ }^{159}$ Gd-DTPA-BMA function as alternative to conventional radiotherapy, owing to the capability of holding ${ }^{159} \mathrm{Gd}-D T P A B M A$ complex and eluding premature release.

Several studies have focused radiosensitization effects of nanoparticles to body through the irradiation externally from machine. However there are few reports on interstitial radiotherapy owing to their potential adverse effects. It is equally important to design strategy for efficient use of nanomedicine for effective radiation outcome by reducing the dosage in interstitial radiotherapy. Mesoporous silica 
coated $\mathrm{Bi}_{2} \mathrm{~S}_{3}$ nanoparticles (BMSNs) loaded with chemotherapeutic drug Dox was developed by Ma et al and demonstrated the effects of radiosensitization of BMSNs in X-ray external beam and P-32 radionuclide interstitial radiotherapy [74]. Bismuth based nanoparticles is currently grabbing considerable attention in radiotherapy owing to its significant enhancement of radiation dosage under kilovoltage $\mathrm{X}$-ray beams than popular gold radiation sensitizer. The researchers observed 45-wt \% loading efficacy of Dox and stimuli responsive ( $\mathrm{pH}-$ ) controlled Dox release and improved therapeutic efficacy against multidrug-resistance cancer cells than free Dox. Nanoparticles along with X-ray treatment resulted in improved therapeutic effects when compared to X-ray beams therapy and augmented therapeutic effects of interstitial P-32 radionuclide radiotherapy in the solid tumour and it was systematically demonstrated both in vitro and in vivo studies. Under in vitro studies, the effect of cellular inhibition of P-32 interstitial radiotherapy along with nanoparticles was 1.55-fold higher when compared to P-32 treatment alone. It was observed that the combination of chemo- and interstitial radiotherapy with BMSNs elevated the therapeutic effects compared to single treatment modality. In vivo studies of P-32 radiotherapy along with BMSNs demonstrated considerable reduction of tumour volumes (21\% reduction) when compared to P-32 treatment alone. The study advocates the application of multifunctional nanosystem directing the efficient co-delivery of anticancer drugs and $\mathrm{Bi}_{2} \mathrm{~S}_{3}$ radiosensitizers to accomplish superior therapeutic goal.

The progress of research in nanotechnology has facilitated the design of versatile nanostructures with theragnostic properties and their application as nanocarriers for emitting radionuclides for internal radiation therapy that aids for treatment of several metastatic abdominal cancers. Incorporation of stable isotopes within a carrier and irradiating a neutron flux is an efficient method for the generation of radioactive agents for radiotherapy. However carrier should be stable and strong enough to tolerate neutron irradiation background, which generates high-localised heat. Several nano carriers for stable isotopes with neutron activation for development of radiotherapeutic agents have been reported. ${ }^{166} \mathrm{Ho}$ is an ideal radionuclide for radiotherapeutic studies. Polymeric microparticles with ${ }^{166} \mathrm{Ho}$ and nanomaterials containing stable ${ }^{165} \mathrm{Ho}$ for neutron activation to ${ }^{166} \mathrm{Ho}$ have been studied [75]. ${ }^{166} \mathrm{Ho}$ is an ideal candidate for radionuclide therapy owing to its high-energy emission of $\beta$-particles (maximum energy of $1.84 \mathrm{MeV}$ and half-life of $26.8 \mathrm{~h}$ ).
Furthermore, ${ }^{166} \mathrm{Ho}$ emits $81-\mathrm{keV}$ y-rays with $6.6 \%$ photon yield that is exploited for both quantitative and qualitative biodistribution. However, polymeric carrier materials that were investigated could survive merely short neutron irradiation without getting degraded or forming aggregates. Di Pasqua and co-workers reported the development of lipophilic acetylacetonate complex of ${ }^{165} \mathrm{Ho}\left[{ }^{165} \mathrm{Ho}(\mathrm{AcAc})_{3}\right]$ in mesoporous silica nanoparticles and irradiation in a neutron flux to generate particles comprising ${ }^{166} \mathrm{Ho}$ through an $(n, \gamma)$ reaction [76]. The ${ }^{166} \mathrm{Ho}-\mathrm{MSNs}$ delivered therapeutic doses for metastases in ovarian cancer after intraperitoneal delivery. The study reports the stability of ${ }^{166} \mathrm{Ho}-\mathrm{MSNs}$, where ${ }^{166} \mathrm{Ho}$ generated by neutron irradiation of ${ }^{165} \mathrm{Ho}-$ mesoporous silica nanoparticles was held in the nanoparticles even after $18 \mathrm{~h}$ of neutron irradiation and was not discharged to the medium after dilution. Tomographic images suggest that radionuclide would probably retain within the mesoporous silica matrix after the administration to peritoneal cavity and selectively hoarded in the tumours.

Radiation therapy comprising brachytherapy is often considered as the monotherapy for early stage cancer or as combination therapy in advanced stage of cancer. Brachytherapy spacers offer the prospect for in-situ delivery of drugs through minimally invasive radiation therapy procedures. Several chemotherapeutic drugs function as radiosensitizers [77]. In such cases, chemotherapeutic drug loaded spacers improves therapeutic ratio of brachytherapy procedure by distributing radiosensitizer to tumour site without systemic toxicity. The systemic administration of chemotherapeutic drugs have numerous disadvantages including fast pharmacokinetics, high systemic toxicity, inadequate periods of radiosensitization which are not coordinated with the dosage of radiation dosage. However use of nanoparticles doped spacers for drug delivery offers several advantages over conventional systemic delivery [78, 79]. It has been established that the use of nanoparticles offer slow and sustained release of drugs, accelerates targeted delivery of therapeutics in high dose to tumour, possibility for the integration of diagnostic moieties, superior spatial distribution of the drug in the tumour and reduced systemic drug toxicities to non-targeted tissue/organs. Kumar et al demonstrated the possibility to construct 'dual' release brachytherapy spacers without intravenous delivery, which had the capability of in-situ release of drug eluting nanoparticles to target tissue via slow and sustained fashion [80]. Researchers fabricated Implantable Nanoplatforms or Chemo-Radiation Therapy (INCeRT) spacers loaded with silica nanoparticles 
(SNPs) with drug for simultaneous localized chemo-radiation therapy. The matrix of INCeRT spacers was prepared from biodegradable poly(lactic-coglycolic) acid (PLGA) which was the same size of commercial brachytherapy spacers (5 $\mathrm{mm} \times 0.8 \mathrm{~mm})$. Cy7.5 was employed as drug and incorporated to silica nanoparticles of $250 \mathrm{~nm}$ in size. The spacers were then doped with docetaxel and studied the stability, in vivo biocompatibility, and biodegradation of spacers in mice models. Diffusion of nanoparticles from the spacer to neighbouring tissue was observed under in vivo imaging whereas control (Cy7.5-PLGA spacer) exhibited fast degradation in a few days along with the burst release of Cy7.5. Spacers loaded with docetaxels displayed tumour suppression as opposed to control mice over 16 days. This study sets a platform for the application of brachytherapy spacers for localized drug delivery in conjunction with radiation therapy.

\section{Magnetic hyperthermia}

Magnetic nanoparticles are versatile class of nanomaterials that has an extensive range of applications both in materials science and biomedical science. Few of its clinical application include magnetically targeted drug delivery, magnetic fluid hyperthermia, magnetic cell separation, MRI, etc. Thermal therapy has garnered immense attraction owing to minimally invasive therapy in current cancer treatment and tumour cells are more vulnerable to high temperature than normal cells [81]. Hyperthermia treatment for cancer is based on application of heat at tumour tissues that is targeted for inhibition of cellular proliferation, regulation and inducing apoptosis. Magnetic hyperthermia for cancer treatment is one of the promising biomedical applications of magnetic nanoparticles. It is a procedure where magnetic nanoparticles are used to induce localized heating to $42-46^{\circ} \mathrm{C}$, to kill tumour cells. Therapeutic effects of hyperthermia were documented directly with magnetic nanoparticles in cancer cells and indirectly by augmenting the effect with chemo or radiotherapy. Magnetic nanoparticles (either plain or encapsulated) function as thermal seeds during magnetic hyperthermia treatment of cancers under an alternating magnetic field. When magnetic nanoparticles are introduced to subjects with tumour in an alternating magnetic field, temperature inside the tumour would rise. The specific absorption rate (SAR) of nanoparticle in an alternating magnetic field, determine the heating rate and thermal energy is released to the surrounding cells thereby destroying cancer cells. MHT is based on the ability of magnetic nanoparticles to dissipate magnetic field energy into heat energy in presence of an alternating magnetic field (AMF). Multifunctional magnetic composite materials have been successfully designed for generating tunable magnetic systems for magnetic hyperthermia to modify intrinsic magnetic properties and to protect them within a shell, in which drugs can be loaded, thus serving dual modalitychemotherapy and magnetic hyperthermia. Magnetic hyperthermia often functions as an adjunct to improve chemotherapy. The localized heat generated by magnetic nanoparticles under external AMF can considerably stimulate drug release and elevate the temperature of the surrounding media $41-46^{\circ} \mathrm{C}$ for potential clinical hyperthermia levels for cancer therapy applications. Although the mechanisms responsible for enhanced drug mediated cellular cytotoxicity associated with magnetic hyperthermia are not completely elucidated, the synergistic action of drug and magnetic heating (enhanced intracellular uptake of drugs owing to increased cell membrane permeability during magnetic heating and disruption of cellular cytoskeleton thus inducing apoptosis) could be some of the reasons for the reduced cancer cell viability [82].

The stability presented by mesoporous silica matrix and potential synergy between the heat production and controlled release of chemotherapeutic agents marks the integration of magnetic nanoparticles with mesoporous silica nanoparticles for the design of nanocomposites for magnetic hyperthermia. Biocompatible nanocomposites of maghemite embedded in mesoporous silica-matrix to develope magnetic microspheres, and the capacity to perform magnetic hyperthermia upon exposure to AMF was reported by Martín- Saavedra et al [83]. Nanoparticles were proficiently internalized by several human cancer cells and were localized in cytoplasm. Nanoparticles did not interfere with normal morphology or metabolic functions of cells, signifying the cytocompatibility of nanomaterial. Also, nanoformulation did not impede the endogenous heat-shock response of a HeLa-derived cell line that specifically reports thermal stresses through changes in the action of a stably integrated hsp70B promoter. Activation of hsp70B promoter in cells treated at $44{ }^{\circ} \mathrm{C}$ in an oven and exposed to magnetic hyperthermia were similar. Temperature of the samples that were exposed to magnetic hyperthermia was elevated as the function of concentration of magnetic nanomaterials. Cancer cell viability was reduced considerably owing to the exposure of cancer cells along with magnetic material under AMF. Magnetic hyperthermia studies demonstrate the ability to control the rise in temperature in cell culture environment upon treatment of nanoformulation and 
exposure of AMF, thus generating heat that severely damaging the proliferation of cancer cells.

In a study, carbon-encapsulated magnetic colloidal nanoparticles (MCN@C) was coated with mesoporous silica shells for the development of core-shell structured magnetic mesoporous nanoparticles $\left(\mathrm{MCN} @ \mathrm{C} / \mathrm{mSiO}_{2}\right)$, and rattle-type structured magnetic mesoporous nanoparticles $\left(\mathrm{MCN} / \mathrm{mSiO}_{2}\right) \quad[84]$ The $\mathrm{MCN} @ \mathrm{C} / \mathrm{mSiO}_{2}$ nanoparticles demonstrated augmented magnetic hyperthermia than $\mathrm{MCN} / \mathrm{mSiO}_{2}$ nanoparticles. However $\mathrm{MCN} / \mathrm{mSiO}_{2}$ nanoparticles exhibited high drug loading capacity when compared to $\mathrm{MCN} @ \mathrm{C} / \mathrm{mSiO}_{2}$. Both nanoformulations presented comparable drug release profile under $\mathrm{pH}$ and temperature responsive drug release. Also both type of nanoparticles displayed good magnetic hyperthermia ability; however it was slightly lower than that of MCN@C nanoparticles due to non-magnetic mesoporous silica coating on $\mathrm{MCN} @ \mathrm{C}$ nanoparticles. Among the mesoporous silica coated nanoparticles, $\mathrm{MCN} @ \mathrm{C} / \mathrm{mSiO}_{2}$ presented superior magnetic heating capacity than $\mathrm{MCN} / \mathrm{mSiO}_{2}$, owing to the retention of iron oxide in the $\mathrm{MCN} @ \mathrm{C} / \mathrm{mSiO}_{2}$ nanoparticles during extracting treatment. The nanoformulation was biocompatible and hence the authors advocate that nanoparticles would be ideal for drug delivery and magnetic hyperthermia for cancer therapy.

DNA-capped $\mathrm{Fe}_{3} \mathrm{O}_{4} / \mathrm{SiO}_{2}$ magnetic mesoporous silica for temperature dependent drug release and magnetic hyperthermia was developed by Zhu et al [85]. An anticancer drug-Dox was loaded into ssDNA functionalized mesoporous silica nanoparticles and was capped with complementary ssDNA (cDNA) through the formation of double-stranded DNA (dsDNA). Drug loaded $\mathrm{MMS}_{-} \mathrm{NH}_{2}-\mathrm{dsDNA}$ complexes demonstrated rapid release of drug at 50 ${ }^{\circ} \mathrm{C}$, but slow release profile at $37{ }^{\circ} \mathrm{C}$. Owing to the superparamagnetic behaviour, nanocomposite generated heat upon exposure to an AMF. When an AMF was applied, DNA-capped MMS nanoparticles generated heat by hyperthermia and triggered denaturation of dsDNA capping chains leading to release of drugs. The nanocomposite function as promising multifunctional platform for prospective cancer therapy with temperature controlled drug release and magnetic hyperthermia. In another study, the effect of magnetic hyperthermia was employed to melt double-stranded DNA-linker and triggered the release of mesopore-loaded drug. Ruiz-Hern andez et al developed oligonucleotide modified mesoporous silica, encapsulating superparamagnetic iron oxide nanoparticles and fluorescein [86]. DNA/magnetic nanoparticle conjugates were used to plug the pores of magnetic silica particles upon hybridization of both DNA strands. DNA duplex displayed a melting temperature of $47^{\circ} \mathrm{C}$ that correlated with the higher limit of magnetic hyperthermia treatment. As a result of elevation in temperature, unsealing and release of a mesopore loaded fluorescein was observed. Thus anticancer thermotherapy associated drug release owing to reversible tampering with base pairing of the DNA sequence under alternating magnetic field was successfully demonstrated.

A nanosystem based on iron oxide nanocrystals encapsulated mesoporous silica nanoparticles decorated on the surface with a thermoresponsive copolymer of poly(ethyleneimine)-b-poly(Nisopropylacrylamide) (PEI/NIPAM) with the potential of remotely controlled release of drugs and proteins in response to an AMF has demonstrated by Baeza et al [87]. Polymer decorated exterior functioned as thermo responsive gatekeeper for entrapped drug/protein molecule and retained protein through electrostatic or hydrogen bonds interactions. The nanosystem loads drugs/protein at low temperatures and release them when the temperature increases $\left(35-40{ }^{\circ} \mathrm{C}\right)$ under a magnetic field. The expanded hydrophilic confirmation of thermoresponsive polymer changes to hydrophobic confirmation and shrinks upon temperature rise, thus diffusing payload to the surroundings.

\section{Phototherapy}

Along with the development of other therapies to combat cancer, phototherapy has attracted much interest in recent years owing to the ease and minimal invasiveness. Photodynamic therapy (PDT) and photothermal therapy (PTT) are two distinctive phototherapy strategies that involve light absorption and photosensitizer (PS) to generate reactive oxygen species (ROS) and heat to destroy cancer cells, respectively. In this section we discuss both PDT and PTT mediated by C/S-MSNs.

PDT is centred on the excitation of photosensitizers (PS) by light leading to generation of singlet oxygen $\left({ }_{1} \mathrm{O}^{2}\right)$ and cytotoxic reactive oxygen species (ROS) that has the potential to kill cells by apoptosis and necrosis. There are various types of PSs, comprising porphyrins, chlorines, metal phthalocyanines (MPcs), phenothiazinium compounds, etc. that have been approved for PDT or are under preclinical examination [88]. Although several PS are being studied, most of the PS examined so far has several disadvantages. The hydrophobic nature and formation of aggregates in aqueous environment results in the alteration of photophysical properties and reduces the quantum yield of ${ }_{1} \mathrm{O}^{2}$. The effectiveness of PDT is commonly associated with 
ROS and it is mainly the ${ }_{1} \mathrm{O}^{2}$ that destroys tumour cells being more cytotoxic. Another disadvantage is the non-selectivity of PS in cells. In general, PS should accumulate mostly in tumour cells leaving normal healthy neighbouring cells. Although several modifications has been performed to develop delivery system to incorporate PS in its monomeric form for preserving its activity and to reach the target tissue, owing to reduce in vivo selectivity, only very few studies have been evaluated for clinical applications. These issues can be overcome by efficient encapsulation of PS in nanoparticles by holding the monomeric form without loss of activity. Another advantage of nanoparticles is the mode of accumulation of PS in cancer cells owing to the enhanced permeability and retention (EPR) effect of tumour tissues and also by the active targeting (attachment of targeting ligands). Several types of nanoparticles encapsulating PS, including polymeric, silica-based nanoparticles, have been demonstrated for the application of PTT. Mesoporous silica nanoparticles are versatile class of nanomaterials and their unique mesoporous structure makes it promising for various applications. The controllable sizes, shape, porosity, diversity in synthetic strategy, offering large room for drug payload, and flexibility for surface modification have made silica nanoparticles as an efficient nanovector for cancer therapy and emerged as promising carrier for PDT applications. Mesoporous silica nanoparticles do not release loaded PS immediately before stimuli response and it is permeable to molecular and ${ }_{1} \mathrm{O}^{2}$, owing to its porous matrix.

Most commonly used method for immobilization of PS inside mesoporous silica or on the surface is by non-covalent encapsulation and by covalent linkage of PS to mesoporous nanostructure [49]. Although covalent linkage of PS to mesoporous nanostructure performs efficiently owing to retention of PS in nanoparticles, non-covalent encapsulation is commonly used. Several studies with mesoporous silica nanoparticles have been studied elaborately under in vitro conditions, along with in vivo PDT, however for clinical attempts; further research in this area is required. In a recent study, a nanoprobe based on upconversion nanocomposites to enhance effectiveness of PDT with reduced heating effect and deep tissue penetration has been reported for highly efficient PDT in HER2-overexpressed breast cancer cells [89]. In the nanocomposite, PS chlorin e6 (Ce6) was conjugated covalently to mesoporous silica to augment the efficiency of PDT by reducing the distance of fluorescence resonance energy transfer (FRET) between UCPs and PS. The cytotoxicity associated with Ce6 in cells was reduced owing to the prevention of leakage of Ce6 from nanocomposite. The nanoprobe stimulated the generation of singlet oxygen and improved PDT efficacy under $808 \mathrm{~nm}$ laser excitation. The in vivo studies demonstrated PDT via intravenous injection in tumour-bearing nude mice. The study demonstrated solution against the overheating effect and enhancing the efficacy of PDT with upconversion nanoprobes.

An internal radiation source for deep-seated tumour therapy with nanoconstruct without the application of external light source was demonstrated by Kamkaew et al [90]. To overcome the limitation of limited tissue penetration of light in PDT, a system that employs Cerenkov radiation by using radionuclides to activate PS (Ce6) was developed. Zirconium-89 ( $\left.{ }^{89} \mathrm{Zr}, \mathrm{t}_{1 / 2}=78.4 \mathrm{~h}\right)$ with higher-energy $\beta$ emitters $(909 \mathrm{keV})$ was used as CR source to activate Ce6 to generate ROS. Hollow mesoporous silica nanoparticles were used as the nanocarrier to encapsulate ${ }^{89} \mathrm{Zr}$ isotope and $\mathrm{Ce} 6$ molecules. The nanoconstruct- $\left[{ }^{89} \mathrm{Zr}\right]-$ HMSN-Ce6 performed efficiently as photo-mediated tumour cell destruction agent both in vitro and in vivo models. (figure 9) Dose-dependent cell cytotoxicity as a function of concentration of PS and ${ }^{89} \mathrm{Zr}$ was observed in in vitro studies. Inhibition of tumour growth was observed when mice were subcutaneously injected with nanoconstruct and histological analysis of tumour section presented impairment to tumour tissues, indicating that reactive oxygen species facilitated cellular toxicity. Radiolabeled [ $\left.{ }^{89} \mathrm{Zr}\right] \mathrm{HMSN}-\mathrm{Ce} 6$ nanoconstruct also functioned as PET image-guided PDT.

The design of stimuli-responsive controlled drug delivery systems extremely significant to augment anti-cancer effectiveness and to lower the potential side-effects in cancer therapy. Such a nanosystem remains closed at normal condition thus preventing leaching of drug and open upon stimuli to release cargo to perform cytotoxic action. The gatekeeper molecules are being used to cap the mesopores of mesoporous silica based nanoparticles for stimuli responsive drug delivery applications. A dual $\mathrm{pH}$-responsive drug delivery system for combined chemo-photodynamic therapy was developed by Yao et al [91]. The nanoparticles respond to extracellular and intercellular $\mathrm{pH}$ stimuli in cancer cells. Acid sensitive PEGylated tetraphenylporphyrin zinc was prepared with cis-aconitic anhydride (CA) (Zn-Por-CA-PEG) and the surface of MSNs was attached to histidine (MSN-His). In the nanoconstruct, at $\mathrm{pH}$ 7.4, Zn-Por-CA-PEG functioned as a gatekeeper to cover the nanopores of MSN-His and prevented leaching of loaded drug. At $\mathrm{pH} 6.8$, acid sensitive cis-aconitic anhydride (CA) in the nanoconstruct 


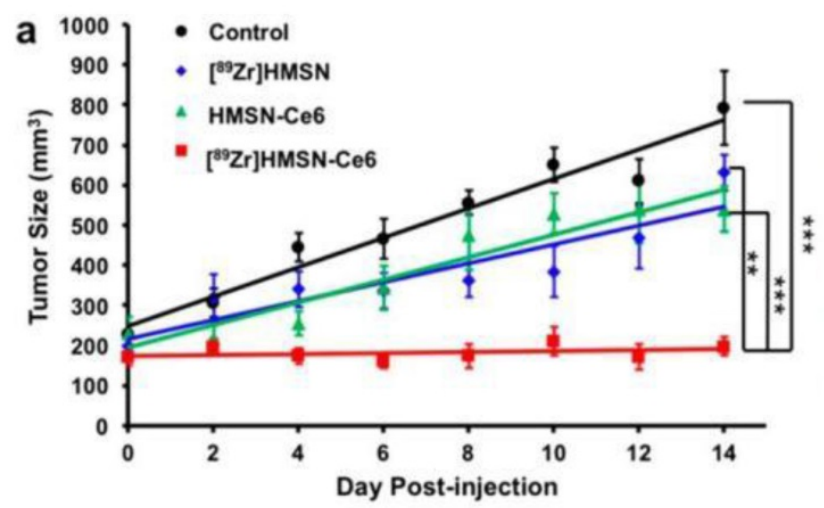

b

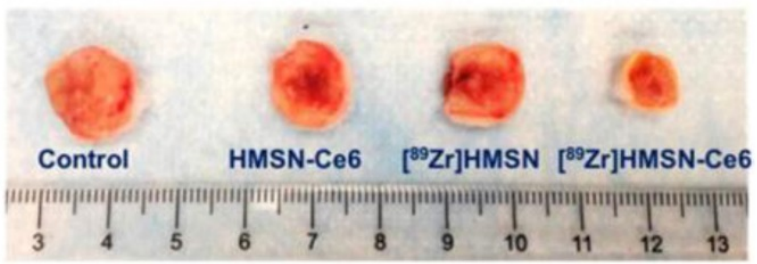

C
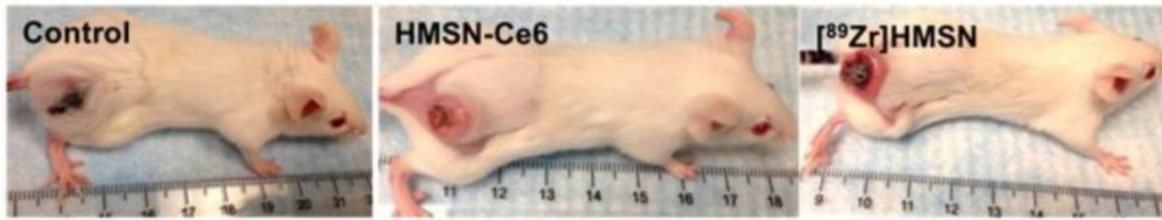

[89 Zr]HMSN-Ce6

d
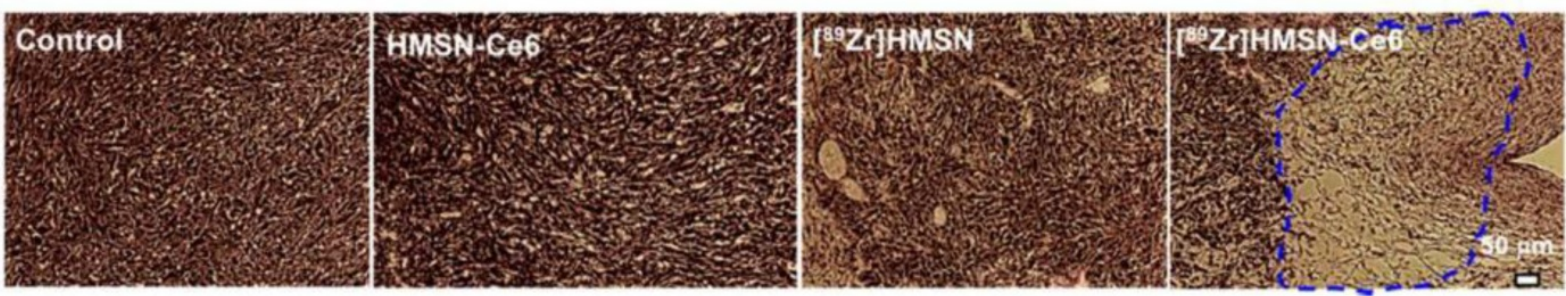

Figure 9: in vivo CR-induced PDT. (a) Tumor growth curves of different groups of mice after various treatments indicated: control group with no injection (black) mice injected with [ ${ }^{89} \mathrm{Zr}$ ] HMSN (blue), HMSN-Ce6 (green), and [ ${ }^{89} \mathrm{Zr}$ ] HMSN-Ce6 (red), with $\mathrm{n}=4$. (b) Representative photographs of tumors from different groups taken at the day 16 p.i. (c) Representative photographs of mice from different groups taken at the day 14 p.i. (d) H\&E-stained tumor slices collected from different groups of mice 7 days after various treatments [103]

cleaved to Zn-Por and PEG. Positively charged Zn-Por assisted cellular internalization and promoted accumulation of drug-loaded MSN at cancer site. As an efficient photosensitizer Zn-Por produced ROS upon irradiation of light thus inducing severe toxicity in cancer cells. The synergistic action of chemo agents and PDT was effective in ablating MCF breast cancer cells. In another study PS loaded mesoporous silica nanoparticle was modified by PEG to improve biocompatibility and PEI to facilitate endosomal escape and to enhance PDT. The second generation PS- zinc (II) phthalocyanine ( $\mathrm{ZnPc}$ ) was loaded into mesoporous silica nanoparticles. Nanoparticles were distributed in the cytosol owing to proton sponge effect offered by PEI, thus augmented the escape efficiency from lysosome. It was observed that average life span of mice in control groups was 16 days, whereas the mice survived over 40 days when treated with nanoconstruct followed by exposure of light demonstrating the efficiency of PDT.

PDT can be used for treatment of deep-seated tumour by employing NIR irradiation owing to the advantage of the optical diagnostic window of tissue (600-1000nm) for deep tissue penetration. Nanomaterials that have absorption in NIR region are highly suitable to meet diagnosis and treatment of deep-seated tumour. Upconversion nanoparticles (UCNP) are examples and in recent times, several studies have been reported on the application of PDT. In a study, mesoporous-silica-coated $\mathrm{NaYF}_{4}: \mathrm{Yb} / \mathrm{Er}$ upconversion nanoparticles was used for NIRfacilitated PDT. A biocompatible photosensitizer, Vitamin B12 (VB12) was integrated into mesoporous silica and served as PDT drug. Up on NIR irradiation, VB12 absorbed fluorescent emission from UCNP under NIR irradiation and excited VB12 molecules generated ROS by interacting with ground-state molecular oxygen, and resulted in the oxidative damage of cancer cells [92]. In another study with upconversion nanoparticles, researchers synthesised UCNPs/silica core-shell structured nanoparticles for PDT and imaging of THP-1 macrophages [93]. The study was aimed as therapy for atherosclerosis. Formation of atherosclerotic plaques is a hallmark of atherosclerosis and around $20 \%$ of cells in atherosclerotic lesion comprise of macrophages. PS 
was grafted inside mesoporous silica shell through covalent interaction. Upon NIR irradiation, light was converted into visible by UCNPs that was adsorbed by PS to generate singlet oxygen. The singlet oxygen mediated cytotoxicity of THP-1 macrophages thus preventing the progress of atherosclerosis.

Photothermal therapy (PTT) is an extension of photodynamic therapy that uses electromagnetic radiation for the treatment of many medical conditions, including cancer. Nanoparticles with PTT capabilities have garnered great attention in the photothermal treatment of tumour. Advantages of PTT include good specificity, nominal invasiveness and accurate spatial temporal selectivity in cancer therapy over conventional therapy [94]. Several inorganic nanomaterials, such as noble metal nanoparticles, carbon nanomaterials, transition metal sulfide/oxides nanomaterials, organic nanomaterials, conductive polymers-based nanomaterials have been reported to generate photothermal heating by NIR irradiation to kill cancer cells [94, 95]. Therapeutic potential of PTT is influenced considerably by the conversion of light energy to adequate heat energy with photothermal nanoscale materials. In cancer therapeutics, PTT is employed as such or in combinatorial approaches along with chemo or PDT to augment the therapeutic potential and multimodal imaging, thus serving as potential theragnostic nanoagents.

PTT using noble metal silica is extensively studied and the first report on photothermal therapy on Au-silica nanoshells was by Hirsch et al [96]. In the nanoconstruct, silica cores were developed to which a thin overlay of $\mathrm{Au}$ was deposited. In general when metallic nanoparticles are irradiated with light at their resonance wavelength, a synchronized oscillation is generated by the conduction-band electrons of nanoparticle that eventually ends in either scattering or absorption of light. Scattering of light relative to absorption of light can be optimized for the projected application by the design of Au nanoparticles based on its composition, size, shape, etc. For tumour hyperthermic applications, Au nanoparticles absorb incident optical energy and transfer to heat energy thus increasing the temperature in tumour cells and ablate tumour [97]. The resonance of gold nanoshells can extent from visible to NIR of the spectrum by tuning the size and thickness of silica core and gold shell respectively. Under in vitro study, human breast carcinoma cells incubated with nanoshells exhibited enhanced photothermal morbidity up on exposure to NIR light.

In recent times, therapeutic potential of PDT is further augmented by integrating with other treatment modalities like photothermal therapy
(PTT). The synergistic effect of loading of Ps to NIR absorbing photothermal nanoparticles like $\mathrm{Au}, \mathrm{C}, \mathrm{Pd}$ nanomaterials have been reported both in vitro and in vivo under NIR irradiation. Hollow silica nanospheres were used to load phthalocyanine with good water-dispersion, and the nanoconstruct (Pc@HSNs) exhibited efficient PDT and PTT effects [98]. Owing to hydrophobicity of phthalocyanine, although it displays significant adsorption at NIR region, the PTT potential was not studied widely. The researches prepared HSNs as nanocarriers to loading phthalocyanine as a model sensitizer and evaluated the in vitro and in vivo PTT and PDT effect. Pc@HSNs converted light energy into heat and generated ROS after laser irradiation thus ablating cancer cells and in tumour bearing mice without significant toxic effects after treatment. Dual phototherapeutic properties arising from a single nanoconstruct thus employing hydrophobic photosensitizers in hollow silica nanomaterials play a major role in cancer treatment.

\section{Gene therapy}

In recent years, gene therapy has evolved as a potential treatment approach in oncology due to the close genetic association between progression and development of tumour. Owing to the inability of naked DNA to get across the cell membrane, gene therapy demands efficient delivery of gene into cells. In general gene delivery vectors are broadly categorized into viral vectors and non-viral vectors. Although viral vectors are efficient, their safety including immunogenicity and chance of gene recombination remains a challenge. Most of the non-viral gene delivery strategy is based on chemical methods that include cationic compounds, recombinant proteins, or polymeric or inorganic nanoparticles [99, 100]. Several studies have confirmed that therapeutic efficiency was enhanced through nanoparticle mediated delivery of gene in cancer therapy. Polymers, cationic lipids, mesoporous silica nanoparticles, carbon nanostructures have been extensively studied under non-viral vector category in the recent times owing to their biocompatibility, improved bio distribution and enhanced efficacy. The distinctive structural characteristics of mesoporous silica nanoparticles bestow them with superior performance in the co-delivery of drug/gene into cancer cells. The porous structure of mesoporous silica nanoparticles permits binding of nucleic acids on the surface and also efficient encapsulation of large amount of drugs within the particles.

Multidrug resistance (MDR) is one among the major complications in oncology with conventional chemotherapeutic agents [101]. Gene therapy function 
as an attractive platform to overcome MDR by the delivery of siRNA to silence specific proteins and to attain effective therapeutic outcome [102]. The knockdown and down regulation of proteins involved in MDR in cancer cells including P-glycoprotein (for pump mechanism) and Bcl-2 (for non-pump mechanism) can improve therapeutic effect for treatment of cancer [103]. Pump and non-pump resistance is instigated when anticancer drugs initiate apoptosis and these resistances must be repressed to enhance chemotherapy efficacy. Combinatorial treatment by delivery of siRNA and chemotherapeutic agents has established enhanced chemotherapy efficiency [104].

Ma et al reported the development of integrated hollow MSNs for target co-delivery of Dox/siRNA against $\mathrm{Bcl}-2$ protein to overcome MDR in cancer cells for cancer therapy [105]. The nanovector with perpendicular nanochannels connected internal hollow cores doubled the drug loading capacity and exhibited $\mathrm{pH}$ responsive Dox release that mimimized pre-release of the same. Mesopores were blocked with folic acid conjugated polyethyleneimine (PEI-FA) on the surface of hollow mesoporous silica nanoparticles and folic acid functioned as targeting ligand that aided the nanovector to enter into the targeted cancer cells. Enhanced therapeutic efficacy was reported by the co-delivery of Dox and siRNA against the Bcl-2 protein into HeLa cells, by effective silencing of anti-apoptotic protein Bcl-2. Meng et al. reported mesoporous silica nanoparticles with polyethyleneimine-polyethyleneglycol (PEI-PEG) copolymer modified surface to overcome Dox resistance in MDR human breast cancer xenograft by the co-delivery of Dox and siRNA which target $\mathrm{P}$ glycoprotein (Pgp) drug exporter [104]. The dual delivery system exhibited synergistic inhibition of tumour growth in vivo and analysis of xenograft biopsies confirmed knockdown of Pgp at heterogeneous tumour sites that correlated with the regions where Dox was released inside the cells and induced apoptosis. Recently Meiying Wu et al reported the development of large pore sized HMSNs and demonstrated the advantage of large pore-sized HMSNs for assisting encapsulation of Dox and P-glycoproteins (P-gp) modulator siRNA to reverse MDR of breast cancer cells both in vitro and in vivo [106]. Stability difference-based selective bond breakage (SD-SBB) strategy was utilized for the development of hollow mesoporous organosilica nanoparticles (HMONs) with enlarged pore size of $\approx 24.0 \mathrm{~nm}$ with hollow interior and organic-inorganic hybrid structure. The concept of large pore size was based on the distinction in chemical-bond stability within the framework where Si-C bond was weaker than $\mathrm{Si}-\mathrm{O}$ bond and the selective breakdown of $\mathrm{Si}-\mathrm{C}$ bonds that resulted in large pore size in the shell of HMONs. A redox-responsive release of gene was attained by the attachment of poly ( $\beta$-amino esters) (PAE) through disulfide linkage. HMONs-ss-PAE, facilitated synergistic effect by the codelivery of P-glycoproteins (P-gp) modulator siRNA by down-regulation of overexpressed P-gp and Dox to revert P-gp mediated MDR of cancer cells. The synthetic strategy of large pore in mesoporous nanomaterials offers a promising nanoplatform for the delivery of large guest molecules. Kim et al reported the synthesis of mesoporous silica nanoparticles with very large pores $(>15 \mathrm{~nm})$ and used them for the delivery of plasmid DNA to human cells [107]. Mesoporous silica nanoparticles with large pores protected plasmids from nuclease-mediated degradation. Mesoporous silica nanoparticles exhibited biocompatibility, high loading capacity of pDNA and proficient cellular uptake. The pDNA loaded nanoparticle exhibited superior transfection efficiency of plasmids encoding luciferase and green fluorescent protein ( $\mathrm{pLuc}$, pGFP) than DNA loaded into mesoporous silica nanoparticles with small pores $(\sim 2 \mathrm{~nm})$.

A degradable, biocompatible nanoparticle by grafting "self-catalyzed degradation" poly(2dimethyl-aminoethyl acrylate) PDMAEA onto the surface of large pore and cubic mesostructured mesoporous silica nanoparticles as a potent gene/drug delivery vector was developed by Hartono et al [108]. PDMAEA was linked covalently to the surface of mesoporous silica nanoparticles with large pores and mediated the binding and release of siRNA 'on-demand'. After endocytosis, siRNA was released from degradable PDMAEA. The co-loaded chloroquine assisted the escape of siRNA from endosome. Functional siRNA (PLK1) and chloroquine was loaded to nanovector that inhibited the viability of KHOS cancer cells considerably. A porous core-shell silica nanocarrier with block copolymers for enhanced delivery of siRNA was designed by Moller et al [109]. The polymer was attached to outer surface of mesoporous silica nanoparticles particles to prevent premature release of siRNA and to permit endosomal escape after transfection. Core- shell nanoparticles with medium-sized pores of $4 \mathrm{~nm}$ exhibited extremely high loading capacity of $380 \mu \mathrm{g}$ per mg siRNA/MSN. An extremely low concentration ( $2.5 \mu \mathrm{g}$ MSN containing $0.5 \mu \mathrm{g}$ siRNA per $100 \mu \mathrm{L}$ well) of nanovector mediated efficient down-regulation of GFP-luciferase fusion reporter protein up to $80-90 \%$.

Hollow silica NPs owing to large outer shell surface areas are considered as prominent nanovectors for the co-delivery of drug and gene 
when compared with the solid counterparts. Most of the gene/drug co-delivery reports on MSNs are based on spherical morphology. In a recent study by Zhao et al reported star like hollow mesoporous silica nanoparticles as a novel nano-platform for redox-triggered multifunctional nanoparticles for synergistic chemotherapy and gene therapy [110]. To attain controlled drug release, stimuli-responsive components as smart gatekeepers on the surface of silica nanoparticles researchers introduced $\beta$-cyclodextrin ( $\beta$ - $\mathrm{CD}$ ) and adamantine (Ad) supramolecular nanovalves. Disulfide bonds were employed as redox-responsive nanovalve to stimulate drug release owing to the abundance of glutathione (GSH) in cancer cells. Thus CD-PGEA gene vectors (comprising of $\beta$-CD cores and ethanolaminefunctionalized poly(glycidyl methacrylate) arms) were presented on the outer surfaces of star shaped hollow silica nanoparticles (SHNPs) with disulfide bond-linked Ad guests which functioned as redox-responsive gatekeepers in the cavities of SHNPs to control drug/gene co-delivery. SHNP-PGEA carriers with six sharp horns demonstrated superior cellular uptake, drug release profile, transfection efficiency, and synergistic antitumor activities. Multifunctional SHNPs was reported as promising candidates for the co delivery of drug/gene for efficient cancer treatment.

\section{Immunotherapy}

Immunotherapy is an expanding biomedical field that holds immense potential for the treatment of disorders including infectious diseases, inflammatory diseases, autoimmune diseases and cancer. The field of immunotherapy has flourished with the wealth of therapeutics including small molecules, peptides and proteins, monoclonal antibodies and cellular therapies. The major goal of immunotherapy in oncology is to enhance immune response to annihilate cancer cells by attracting immune cells $\left(C D 8^{+} \mathrm{T}\right.$ cells and natural killer cells) at the tumor site. There are several approaches for immunotherapy including cancer vaccines that may ultimately enhance effector-cell survival and activation, transfer of tumor-reactive $\mathrm{T}$ cells and use of exogenous chemokines to escalate the infiltration of immune cells into tumor to stimulate tumor- specific immune response.

Considering the significance of immunotherapy in oncology, there are over 10 therapeutic monoclonal antibodies approved that targets B-lymphocyte antigen (CD20), vascular endothelial growth factor (VEGF), CD52, and CD33, receptor tyrosine protein kinase erbB-2 in breast cancers (HER2) [111]. Immunotherapy integrated along with other treatment modalities for example chemotherapy has shown to enhance the therapeutic potential [112]. Recently designed virus-like particles presented immunostimulatory capabilities for metastatic cancer [113]. However challenges associated with targeted delivery and controlled release are limiting the efficacy of immunotherapy. Furthermore, proliferation of immunosuppressive cells, enhanced production of immunosuppressive cytokines, reduced immunogenicity of tumour antigens limit the antitumor response stimulated by immunotherapies [114]. Systemic toxicity is induced by the nonspecific delivery of monoclonal antibody and proinflammatory cytokines.

Recently nanoparticles are been widely exploited as potential carriers for the delivery of immunotherapeutics. Mesoporous silica nanoparticles have the potential to incorporate most of functionality of interest and special structural characteristics have been implemented for targeted anti-cancer therapies and vaccines [115]. Mesoporous silica nanoparticles based adjuvants have demonstrated to elicit potent anti-tumour responses [116]. Heidegger et al demonstrated immune response to functionalized mesoporous silica nanoparticles [117]. The silica nanoparticles exhibited biocompatibility in primary immune cells from spleen. A strong immune response was elicited when surface-functionalized mesoporous silica nanoparticles, coated with a $\mathrm{pH}$ responsive polymer and loaded with an immune response activating drug (synthetic Toll-like receptor 7 agonist R848). However the immune response was less when primary cells were treated with cargo free mesoporous silica nanoparticles. Controlled release of immune-activating drug from MSN for localized immune activation in the tumour microenvironment without systemic adverse effects was attained.

Recently Zheng et al demonstrated the development of sub-100 nm sized amine magnetic mesoporous silica nanoparticles (M-MSN-A) modified with PEG as nanovectors for the delivery of CpG-ODN [118]. Magnetic core offered magnetically targeted guidance to the nanovector by applying an external magnetic field. The nanovector demonstrated high loading capacity of $\mathrm{CpG}$ and was biocompatible. When combined with chemotherapeutic drugs, nanovector activated macrophages and inhibited tumour cells. In in vivo models, nanovector demonstrated exceptional immuno-stimulating activity when compared to free $\mathrm{CpG}$ therapeutics. Lee et al demonstrated immunological approaches to enhance host immune response for cancer treatment [119]. They developed folic acid (FA)/secondary lymphoid tissue chemokine (CCL21)/upconversion fluorescent nanoparticles (UCNs) nanoconjugates as a 
targeted nanodelivery system to recruit immune cells $\left(\mathrm{CD}^{+} \mathrm{T}\right.$ cells, $\mathrm{CD}^{+} \mathrm{T}$ cells and dendritic cells) to folate receptor (FR) expressing ovarian carcinoma cells (OVCAR-3 cells). The nanoconjugate proficiently crossed endothelial cell monolayer and gathered in OVCAR-3 cells in endothelial-tumor cell bilayer model. In vitro studies demonstrated that the CCL21 loaded nanoconjugate induced $\mathrm{T}$ cells migration. The study served as proof-of concept, for loading biologically active chemokines in MSN nanosystem to modulate $\mathrm{T}$ cell migration.

\section{Diagnostic applications}

The integration of core shell mesoporous nanoparticles with functional inorganic materials renders contrast properties that are widely exploited for the diagnostic applications. Here we discuss recent progress in the optical imaging with special emphasis on near infra-red imaging, MRI, Positron emission tomography (PET), X-ray computed tomography (X-ray CT), ultra sound with contrast agent incorporated core shell mesoporous silica nanoparticles along with multimodal imaging.

\section{Optical imaging}

Optical imaging is a promising and attractive diagnostic tool in cancer owing to its beneficial properties including non-invasiveness, versatility, enhanced sensitivity, high resolution (submicron spatial resolution and sub millisecond temporal resolution), absence of any ionizing radiation, multimodal detection potential, targeting capabilities and low cost [120]. It plays a significant role to monitor the bio-distribution and metabolism of drug owing to the availability of wide range of contrast agents as nanocarrier for molecular targeting and does not induce deleterious damage to healthy cells or tissues. In optical imaging, fluorescent contrast agents are normally excited by radiation in visible or NIR region of the spectrum, and light with lower energy is emitted than the incident light. In spite of all these exceptional diagnostic potential, optical imaging suffers from few limitations including weak signal to noise ratio owing to autofluorescence by tissues, low penetration, and photobleaching. The advent of nanomaterials has enabled significant enhancement in the quantum yield, photostability, biocompatibility, hydrophilicity, and fluorescent signals for the precise detection of fluorescent signals. In most clinical applications studies, fluorophores in NIR radiation (in the range of $650-950 \mathrm{~nm}$ : diagnostic window) is chosen owing to its greater penetration potential and reduction in the auto fluorescence by blood and body tissue absorption [121, 122] (figure 10). Commonly used fluorophores falls under two major categories organics [Cyanines, Alexa Fluors, infrared dyes, indocyanine green, methylene blue, 1,1'-dioctadecyl3,3,3',3'-tetramethylindodicarbocyanine, 4-chlorobenzenesulfonate salt (DiD), 1,1'-dioctadecyl3,3,3',3'-tetramethylindocarbocyanine perchlorate (Dil)] and inorganics (Quantum dots (QDs), lanthanides). However photobleaching and potential toxicity induced by these fluorophores again limits its in vivo application. Therefore it is crucial to protect fluorescence without compromising safety. Mesoporous silica nanoparticles owing to its extremely high biocompatibility, chemical inertness, and easily modifiable surface are ideal nanomaterials to carry and release the fluorescent cargo for tracking studies by preserving the fluorescence. Fluorophore are either encapsulated, immobilized or adsorbed onto the mesoporous structures and are protected by mesoporous shell. Mesoporous silica nanostructures offer very high surface areas and permit high loading of cargo for targeted drug delivery of drug and cellular imaging. As mentioned above, fluorescence signal of conventional fluorescent dyes (emission in UV or visible range of spectrum) affects auto-fluorescence from biological tissues that result in limited penetration in bodily tissue. To overcome auto-fluorescence effects NIR fluorescent dyes protected with mesoporous silica nanoparticles are employed.

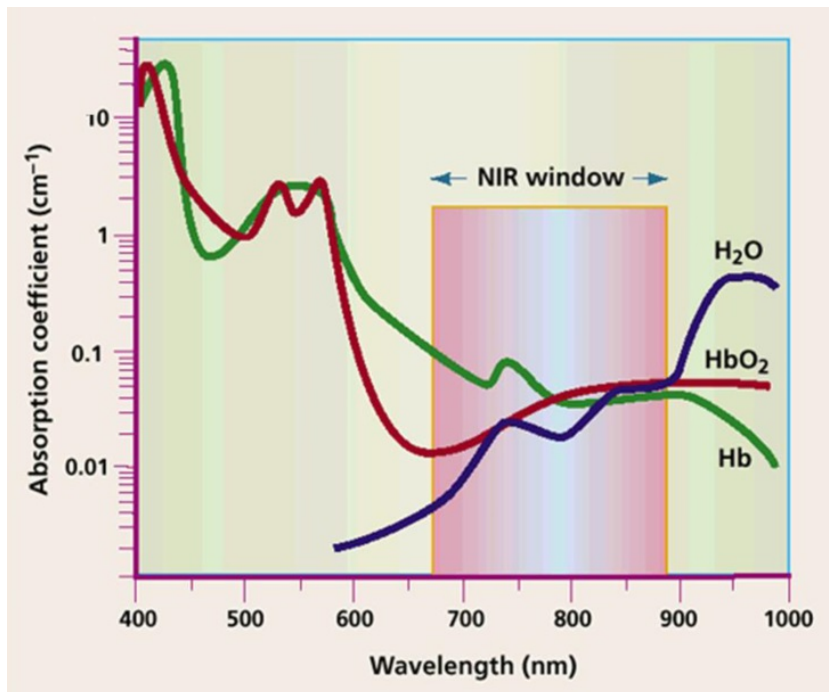

Figure 10: The NIR diagnostic window. Hemoglobin $(<650 \mathrm{~nm})$ and water $(>900 \mathrm{~nm})$ exhibits minimal absorption [134].

Indocyanine green (ICG) is an excellent NIR fluorophore approved by FDA and is widely used in in vivo NIR fluorescence imaging and photodynamic effects in cancer cells [123]. However high background fluorescence during circulation leading to low target-to-background ratio (TBR) in in vivo imaging, nonspecific uptake in cells limits its 
applications for fluorescence imaging and for phototherapy of cancers. Lee et al reported first study of MSN functionalized with NIR-ICG for in vivo optical imaging with high efficiency [124]. Mesoporous silica nanoparticles was modified with trimethylammonium groups to adsorb indocyanine green (ICG) by electrostatic attraction. Stable and strong fluorescence of contrast agent was observed in the liver of anesthetized rat. The reduction in aggregation and quenching of fluorescence may be attributed to the distribution of ICG molecules in the mesoporous silica nanoparticles with very high surface area and nanopores of mesoporous silica protect the dye molecules from degradation.

Zeng et al developed ICG loaded mesoporous silica nanoparticles as fluorescent probe and its role in intraoperative NIR-image-guided liver tumour resection [125]. Arginine-glycine-aspartic acid (RGD)targeted mesoporous silica nanoparticle loaded with ICG dye was synthesized with the potential to demarcate liver cancer cells and to deliver high tumour-to-normal tissue contrast intraoperatively. The nanoprobe identified microtumours and satellite lesions of less than $1 \mathrm{~mm}$ (average size of $0.4 \pm 0.21 \mathrm{~mm}$ ) in living mice owing to optical contrast properties of ICG and establishing prospect for surgeons for accurate elimination of microfoci during liver surgery. A dual modal imaging strategy that combines X-ray computed tomography (X-ray CT) and fluorescence imaging with ICG loaded mesoporous silica-coated gold nanorods was demonstrated by Luo et al [126]. NIR fluorescence of ICG from nanocomposite was observed up to $12 \mathrm{~h}$ post intratumoural injection and X-ray CT scanning from $\mathrm{Au}$ component of nanocomposite exhibited significant contrast enhancement demonstrating the potential for the use in targeted cancer diagnosis in combination with radiotherapy and photothermotherapy.

Xie et al. reported synthesis of mesoporous silica nanoparticle for anti-cancer drug delivery and in vivo bioimaging mediated by Dox and NIR fluorescent dye Cy5 respectively [127]. They developed carboxyl functionalized mesoporous silica nanoparticles and coated with $\mathrm{NH}_{2}$-PEG-folate as the cancer targeting ligand and Cy5 via 1-ethyl-3-(3dimethylaminopropyl) carbodiimide hydrochloride (EDC)/N-Hydroxysulfosuccinmide sodium salt (Sulfo-NHS) coupling as diagnostic moiety. Carboxyl functionalization of silica nanostructure rendered more negative charge in pore surface than native mesoporous silica nanoparticles, thus favouring electrostatic attraction between positively charged Dox and nanoparticles. Folate targeting enabled enhanced cellular uptake of nanoparticles and release of drug in tumour cells than normal healthy cells. NIR fluorescent dye Cy5 in the nanosystem was efficient for both live cell and in vivo imaging demonstrating the ability of mesoporous silica nanoparticles for both effective in vivo imaging and treatment of diseases. Xue and co-workers designed a multimodal-imaging platform with mesoporous silica nanoparticles [128]. They developed fluorescent (NIR dye Cy5.5) dye doped mesoporous silica onto super paramagnetic iron oxide nanoparticles and loaded iodinated oil into mesoporous silica shell and demonstrated its application in MRI/CT/fluorescence imaging. In vitro studies exhibited enhanced fluorescence signal intensity of nanocomposite and accumulation of contrast agent in liver was examined by MRI, CT, and fluorescence imaging in in vivo study.

Chen et al designed hollow mesoporous silica nanoparticle with sophisticated surface modification techniques for efficient in vivo tumour targeting; multimodal imaging, bio-distribution and enhanced delivery of antitumour drugs [129]. They conjugated targeting moiety (anti-CD105 antibody), NIR dye (ZW800) and PET isotope $\left({ }^{64} \mathrm{Cu}\right)$ on to the nanostructure for in vivo tumour targeted PET/ NIRF imaging along with delivery of Dox in tumour. (figure 11) An optimal ratio between HMSN and ZW800 of 1:2 was maintained to prevent the self-quenching effect. In vivo NIRF imaging with ZW800 demonstrated higher optical intensity of tumour in mice injected with targeted nanoparticles.

Song et al reported IR-783 loaded mesoporous core shell silica nanoparticles for in vivo imaging [130]. IR-783 is a biocompatible novel water-soluble cyanine dye with enhanced photo stability and fluorescence quantum yield owing to the cyclohexenyl substitution introduced into polymethine linker and a substituted chlorine atom in cyclohexene ring structure. As two sulfonic acid groups in the dye have a strong negative charge, synthesis of positively charged mesopores of $\mathrm{mSiO}_{2}$ can favour electrostatic adsorption of IR-783. Loading capability of the IR-783 to the nanoprobe$\mathrm{Au} @ \mathrm{mSiO}_{2}$-TTA was influenced by surface area, pore volume and thickness of mesoporous silica shell. $\mathrm{mSiO}_{2}$ with thick shell and larger surface area can facilitate higher loading capacity of dye resulting in strong fluorescence intensity and signal-to-noise ratio. However the nanoparticle was intended for multimodal imaging and increased thickness may reduce imaging potential of other imaging strategies, hence $\mathrm{Au} @ \mathrm{mSiO}_{2}$-TTA particle size of moderate thickness was chosen. Thickness of the $\mathrm{mSiO}_{2}$ shell was maintained as $27 \mathrm{~nm}$ and approximate size of 75 $\mathrm{nm}$. Fluorescence from nanoprobe was stable and functioned as in vivo NIR fluorescent probe. The 
half-life of the nanoprobe in blood was $1.5 \mathrm{~h}$, and fluorescence imaging exhibited higher liver fluorescence than spleen fluorescence.

Apart from NIR dye doped mesoporous silica nanoparticles, NIR-light-emitting inorganic nanoparticles (semiconductor quantum dots-QDs, luminescence lanthanides etc) that display superior photo stability, colloidal stability, narrow emission and enhanced blood circulation that makes them favourable for in vivo optical imaging applications. Lanthanide-based fluorescent nanoparticles have features including single-photon and two-photon excitation and upconversion luminescence and down conversion luminescence that are attractive for in vivo bio imaging. Lanthanide doped mesoporous silica nanoparticles are synthesized by integrating lanthanide ions, or by grafting the silica nanoparticles with metal complexes (Eu(III), Tb(III) or $\mathrm{Gd}(\mathrm{III}))$. In most of the studies, other functional elements are integrated along with NIR-light-emitting inorganic nanoparticles to contribute additional imaging modalities including CT, PET, MRI to offer multimodal imaging for tumour diagnosis. Fan et al developed a multifunctional nanotheragnostic nanocomposite of sub-80 $\mathrm{nm}$ rattle-structured Gd-UCNPs core with mesoporous silica shell (UCMSNs) [131]. UCMSNs were successfully used for locating tumours by functioning as contrasting agents in upconversion luminescence (UCL) and magnetic resonance (MR) bimodal imaging. Researchers also demonstrated tri-modal synergistic cancer therapy in one single nanotheragnostic system by co-delivery of a radio-/photo-sensitizer hematoporphyrin (HP), a radio sensitizer/ chemo drug docetaxel (Dtxl).

Recently, $\mathrm{Su}$ et al has developed a tumourtargeted multifunctional mesoporous upconversion nanoparticle-based theragnostic system with the potential for multimodal imaging [132]. Core-shell mesoporous upconversion nanoparticles with superior upconversion luminescence emission property was integrated with $\mathrm{CuS}$ as a gatekeeping agent for preventing premature drug release and facilitating other therapeutic modalities. Attachment of hyaluronic acid on the surface of mesoporous
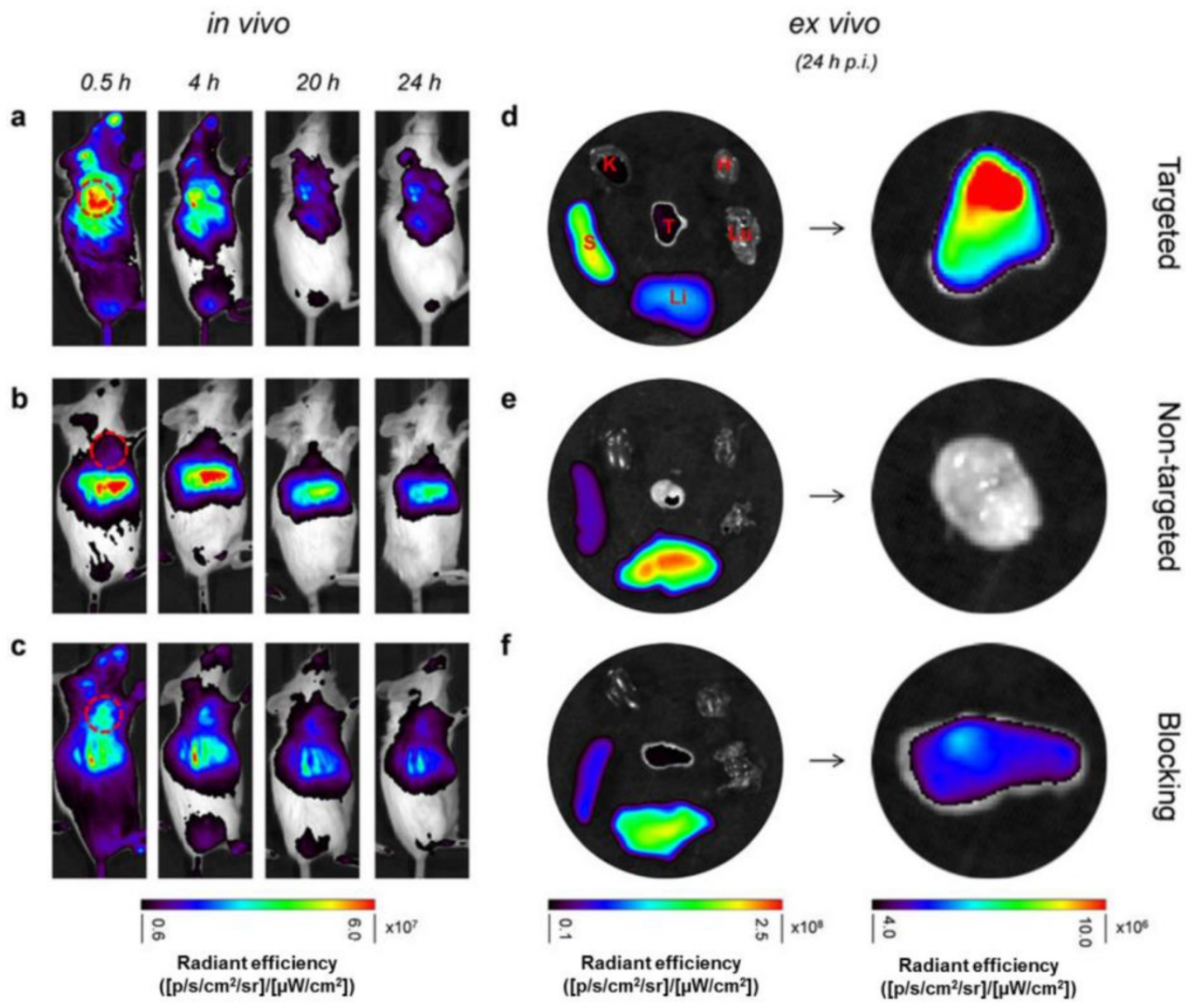

Figure 1 1: in vivo tumor (Tumors were marked by dashed red circles-) targeted NIRF imaging. (a) Targeted group. (b) Non-targeted group. (c) Blocking group. Ex vivo NIRF imaging of major organs (left) and tumor-only (right) from 4T1 tumor-bearing mice at 24 h p.i. (d) Targeted group. (e) Non- targeted group. (f) Blocking group. Kidney $(\mathrm{K})$, spleen $(\mathrm{S})$, liver (Li), lung $(\mathrm{Lu})$, heart $(\mathrm{H})$, tumor $(\mathrm{T})$ [142]. 
UCNPs as a targeting moiety for the interaction of CD44 receptors improved targeted delivery of nanoparticle for enhanced antitumour activity and for imaging. The study also reported the progression of tumour by multi-mode imaging for monitoring the size, site and morphology of tumour. UCL imaging was compared with targeted and non-targeted nanoparticles and it was observed that uptake of samples with HA was more in tumour and reached the tumour faster thus signifying targeting efficiency of mUCNPs@DOX/CuS/HA. Zhao et al developed $\mathrm{Fe}_{3} \mathrm{O}_{4} @ \mathrm{SiO}_{2} / \mathrm{NaYF}_{4}: \mathrm{Yb}$, Er (MSU) hybrids modified with $\mathrm{MnO}_{2}$ nanosheets $\left(\mathrm{MSU} / \mathrm{MnO}_{2}\right.$ ) based on the combination of magnetic nanoparticles and emission of upconversion nanoparticles (UC) [133]. Amino-modified $\mathrm{Fe}_{3} \mathrm{O}_{4} @ \mathrm{SiO}_{2}$ particles was covalently linked to carboxyl-functionalized $\mathrm{NaYF}_{4}: \mathrm{Yb}, \mathrm{Er}$ particles. The nanocarrier was designed based on GSH/ $\mathrm{MnO}_{2}$ redox reaction and a model drug; dye was loaded into $\mathrm{MnO}_{2}$ nanosheet thus serving as drug carriers and quenching UC luminescence. When GSH was introduced, $\mathrm{MnO}_{2}$ nanosheets were reduced to $\mathrm{Mn}^{2+}$ that triggered the release of drug and restoring luminescence that was previously quenched by $\mathrm{MnO}_{2}$ nanosheets. The study demonstrates $\mathrm{MSU} / \mathrm{MnO}_{2}$ as promising nanoplatforms for smart drug delivery system and tracking by UCL.

Mesoporous core-shell structured up-conversion nanoparticles (mUCNPs), comprised of $\mathrm{NaYbF}_{4}: 2 \% \mathrm{Er}$ core and mesoporous $\mathrm{NaGdF}_{4}$ shell endowed with several functionalities including drug delivery and bioimaging capabilities was demonstrated [134]. The nanocomposite was decorated with folate as targeting ligand for enhanced cellular uptake and high uptake was observed in the UCL cell imaging with folate tagged mUCNPs. The core-shell mUCNPs was also as the contrast agents for X-ray computed tomography $(\mathrm{X}$ ray $\mathrm{CT}$ ) owing to strong $\mathrm{X}$-ray attenuation of $\mathrm{Yb}$ and as MR contrast agent due to high longitudinal molar relaxivity $\left(r_{1}\right)$ of $\mathrm{Gd}$ in the nanocomposite, demonstrating multifunctional bio-imaging and targeted cancer therapy.

Sentinel lymph nodes (SLNs) imaging has been considered as a significant approach for non-invasive diagnosis of tumour metastasis. Huang et al reported mesoporous silica-based triple-modal imaging probes with multiple functionalities for NIR, optical, MR and PET imaging [135]. NIR dye ZW800 was integrated along with a MR contrast agent $\left(\mathrm{Gd}^{3+}\right)$ and radionuclide $\left({ }^{64} \mathrm{Cu}\right)$. Long-term in vivo imaging was attained with nanoprobes owing to the high stability and long intracellular retention time. Signal from tumour draining SLNs were demonstrable up to 3 weeks and significant differences was observed in the rate of uptake of nanoparticles and contrast between metastatic and normal contralateral SLNs (N-SLNs). NIR fluorescent silica-coated Au nanoparticle clusters $\left(\mathrm{Au} @ \mathrm{SiO}_{2}\right)$ was demonstrated for its multifunctional imaging capability for the detection of lymph nodes (LN) and lymph vessels (LV) by Hayashi et al [136]. Nanocomposite was endowed with dual imaging modality and both fluorescence imaging by etrakis(4-carboxyphenyl)porphyrin (TCPP) and CT demonstrated nanoparticles was accumulated in LNs through LVs. The fluorescence imaging facilitated the identification of both LNs and LVs.

\section{Magnetic Resonance Imaging}

In recent decades, MRI functions as the most proficient diagnostic probe in clinical research. Owing to its advantages including significant spatial resolution, higher depth penetration rate, intense soft tissue contrast MRI is widely used as a competent non-invasive diagnostic probe in medical arena. In the presence of a magnetic field and radio frequencies, MRI signals are developed from the relaxation of protons in water molecules and other components including lipid, protein, etc to create images with enhanced contrast and high resolution. MR images are acquired from differences in longitudinal relaxation time ( $T_{1^{-}}$spin-lattice relaxation) and transverse relaxation time ( $\mathrm{T}_{2}$ - spin-spin relaxation). Though MRI is significantly used as a diagnostic probe, currently contrast agents including nanoparticles are used to overcome the limitation, especially the low sensitivity associated with conventional MRI. In general $\mathrm{T}_{1}$-weighted images are generated by gadolinium and $\mathrm{Mn}$ based contrast agents for analysis of morphological details. On the other hand $\mathrm{T}_{2}$-weighted images are generated by iron oxide or ferrites based contrast agents and commonly used for gaining fine details of inflammation and edema [137, 138]. Based on the development of effective and highly specified contrast agent loaded nanoparticles, MR imaging technique has become renovated as most competent non-invasive diagnostic tool in clinical imaging field. Among the nanoparticles, mesoporous silica nanoparticles over the contrast agent as shell serve as ideal nanoplatform for upgrading MR nanomaterials. MRI with mesoporous silica nanoparticles can be primarily attained by the incorporation of super paramagnetic iron oxide nanoparticles (SPIONs), gadolinium, or manganese oxide [139-141]. Paramagnetic complexes of $\mathrm{Gd}$ or $\mathrm{Mn}$ ions reduces longitudinal $\left(\mathrm{T}_{1}\right)$ relaxation time by inducing local relaxation change of adjacent water protons and provide positive contrast (bright signal) on $\mathrm{T}_{1}$ - weighted MR image. On the other hand SPIONs have shortened transverse $\left(\mathrm{T}_{2}\right)$ relaxation time and offer negative contrast (dark signal) on 
$\mathrm{T}_{2}$-weighted MR image.

SPIONs are the most commonly studied MRI contrast agents. Most studies reported high $\mathrm{T}_{2}$ contrast of core-shell magnetic mesoporous silica nanoparticles; however the parameters that lead to enhanced contrast was studied by Hurley et al [139]. The influence of size, synthesis of iron oxide nanoparticle core and hydrothermal treatment on the transverse relaxivity $\left(r_{2}\right)$ of magnetic mesoporous silica nanoparticles was studied. Particle size difference imparted by the encapsulation of SPIONs did not influence imaging capability. Non- porous silica coating resulted in reduced $r_{2}$ values owing to reduced interaction of water with the magnetic field of SPION and mesoporous silica nanoparticles was advantages over porous silica nanoparticles. Hydrothermal treatment retained $r_{2}$ for over 30 days in water and offered excellent colloidal stability in acidic conditions and imparted resistance against acidic degradation of super paramagnetic core. It enabled the nanoparticles to function as excellent imaging probes for cancer as many cancers are associated with acidic environment. In several studies mesoporous silica nanoparticles loaded with ultra-small iron oxide nanoparticles has been demonstrated for real time monitoring of drug release [142]. As the loaded anticancer drugs are released in acidic environment of tumour, the magnetic centres (integrated paramagnetic iron centres) in mesoporous nanoparticles are exposed to water molecules and MR signal intensity was enhanced. Multifunctional mesoporous silica nanoparticles are designed and integrated with multiple iron oxide nanoparticles are employed for simultaneous MRI and drug delivery [143].

In a study sub-50 nm multifunctional superparamagnetic mesoporous nanoparticles with surface modified with cyclic nine-amino acid peptide LyP-1 (Cys-Gly-Asn-Lys-Arg-Thr-Arg-Gly-Cys) was developed [144]. Nanoparticles were designed with a core of silica shielded magnetite nanoparticle and a shell of FITC-labeled mesoporous silica. The surface was modified with LyP-1 peptide as targeting ligand. The multifunctional nanospheres were effective in targeting pancreatic cancer cell and resulted in distinct MRI signal reduction under $\mathrm{T}_{2}$ relaxometry in vitro studies. In vivo experiments demonstrated nanoparticles was detected in orthotopic pancreatic cancer in C57BL/ 6 mouse as MR contrast agents. A steady reduction of signal in tumour zone was observed after $1 \mathrm{~h}$ post administration of nanoparticles, and signal touched the lowest intensity at $4 \mathrm{~h}$, signifying selective accumulation of nanoparticles in pancreatic cancer. The reduced signal was maintained even after $24 \mathrm{~h}$, post injection in tumour site signifying the stability of magnetic nanoparticle.

Yang et al developed two-dimensional $\mathrm{WS}_{2}$ nanosheets with self-assembled iron oxide nanoparticles (IONPs) on their surface [145]. The surface of nanosheet was coated with mesoporous silica and functionalized with PEG, resulting in $\mathrm{WS}_{2}$-IO@MS-PEG for multimodal imaging, drug delivery, and for photothermal therapy. IONPs gifted the $T_{2}$ contrast in $M R$ imaging modality to the nanostructure and mesoporous silica shell offered room for loading of Dox. Concentration-dependent darkening effect demonstrating high transverse relaxivity $\left(r_{2}\right)$ of $121.5 \mathrm{mM}^{-1} \mathrm{~s}^{-1}$ was observed in $\mathrm{T}_{2}$-weighted MR images of $\mathrm{WS}_{2}$-IO@MS-PEG. Successful in vivo imaging of 4T1 tumour- bearing mice with prominent contrast was observed in the tumour site owing to the accumulation of $\mathrm{WS}_{2}$-IO@MS-PEG after intravenous administration.

Owing to the large magnetic moment resulting from seven unpaired electrons and comparatively extended electron spin-relaxation time $\left(10^{-9} \mathrm{~s}\right)$ in the magnetic field, Gd ions could shorten longitudinal relaxation time of GdNPs [146]. In most studies Gd chelates, such as Gd-diethylenetriaminepentaacetic acid (Gd-DTPA) are used to reduce the toxicity associated with free $\mathrm{Gd}$ ions. However nanocarriers are designed to efficiently integrate $\mathrm{Gd}$ ions to offer excellent contrast enhancement. A core-shell structured theragnostic mesoporous silica based nanoplatform functionalized by components including photosensitizer Ce6, carbon dots (CDs), and dual modal imaging agent Gd (III) ions for MRI and CT has been designed by Yang et al [147]. Thermo/pH-sensitive polymer poly[(N-isopropylacrylamide)-co-(methacrylic acid)] (P(NIPAm-co-MAA)) was used to encapsulate pores and outer shell of core-shell nanospheres. Final Dox loaded product was labelled as DOX-loaded Gd-Si-Ce6-CDs-P(NIPAm-MAA) [Dox-GSCCP]. Gadolinium $(\mathrm{Gd})$ nuclide was reported to function both as an MRI and CT contrast agent. As the "negative lattice shield effect" (n-LSE) on high Gd ion concentration on the nanoparticle may shorten $\mathrm{T}_{1}$-weighted relaxation time of water protons, in their study in order to increase longitudinal relaxivity, Gd-DTPA complex was attached to amino group on the surface of GSCCP. Concentration-dependent positive contrast enhancement was exhibited by DOX-GSCCP and enhancement in $r_{1}$ value was ascribed to the increased concentration of $\mathrm{Gd}^{3+}$ in the outer shell for strong water proton spin- lattice relaxation effect. The efficiency of $\mathrm{T}_{1}$ - weighted MRI in tumour-bearing mice at the tumour site demonstrated enhanced MR contrast effect of Gd ions 
chelated in the nanoconjugate establishing the potential of $\mathrm{T}_{1}$-weighted Gd MRI contrast.

Huang et al developed multimodal imaging nanoprobe for imaging lymph nodes. Mesoporous silica nanoparticles were labelled with $T_{1}$ contrast agent $\mathrm{Gd}^{3+}$ along with other imaging modalities and long-term in vivo imaging was studied. In the nanoprobe, $\mathrm{Gd}^{3+}$ was chelated by Si-DTTA and presented high $\mathrm{T}_{1}$ signal [135]. The 3D gradient-echo scan (FLASH) images displayed enhanced $T_{1}$ signal in T-SLNs (circle) after MSN-probe injection. The contrast of MSN-probes in T-SLNs was observed up to twenty one days. High spatial resolution and anatomic references from MRI images with the nanoprobe to visualize and map tumour draining SLNs makes them an excellent candidate for visualizing lymph nodes.

Chen et al developed multifunctional nanogated envelope-type nanosystem for $\mathrm{pH}$-responsive drug delivery and MRI [148]. The nanosystem was based on lanthanide doped nanoparticle-capped with mesoporous silica nanoparticles. Ultra-small lanthanide doped nanoparticles $\left(\mathrm{NaGdF}_{4}: \mathrm{Yb} / \mathrm{Tm} @ \mathrm{NaGdF}_{4}\right)$ with ultra-thin TaOx layer were attached onto mesopores with the acid-labile acetals that aided $\mathrm{pH}$ responsive drug release in acidic tumour environment. The nanosystem was further modified with PEG for longer circulation time and to accumulate in tumour and for the visualization by MRI. The gadolinium in the nanosystem functioned as $\mathrm{T}_{1}$-MRI contrast agents and $\mathrm{T}_{1}$-weighted magnetic resonance (MR) images were acquired. MR images of tumour-bearing mice model were studied and $\mathrm{T}_{1}$ signals from tumour sites displayed an increasing pattern and were strong at 3 h. MRI was also used to track metastasis behaviour of tumour with the developed nanosystem demonstrating its potential in diagnostic regime. The integration of $\mathrm{Gd}^{3+}$ complexes is widely reported, however, the in vivo release of metal ion during metabolic processes, even when $\mathrm{Gd}^{3+}$ was chelated by organic ligands can cause toxicity issues. Design of $\mathrm{Gd}_{2} \mathrm{O}_{3}$ coated by mesoporous $\mathrm{SiO}_{2}$ nanoparticles by Shao and co-workers demonstrated an increase in $\mathrm{T}_{1}$ for $\mathrm{Gd}_{2} \mathrm{O}_{3} @ \mathrm{SiO}_{2}$ than Gd-DTPA under in vitro studies [149]. In vivo studies with $\mathrm{Gd}_{2} \mathrm{O}_{3} @ \mathrm{SiO}_{2}$ presented enhanced contrast in xenografted tumour indicating that nanoparticles had penetrated tumour vascular endothelial gaps and accumulated in tumour tissues and MRI signal intensity was observed more than $24 \mathrm{~h}$.

Although gadolinium $\left(\mathrm{Gd}^{3+}\right)$-based $\mathrm{T}_{1}$-positive contrast agents are extensively employed in vivo $\mathrm{MR}$ imaging, the risk of nephrogenic systemic fibrosis associated with Gd limits its in vivo application.
$\mathrm{Mn}^{2+-}$ based $\mathrm{T}_{1}$-positive is safer alternative to $\mathrm{Gd}$ based contrast agents, however the efficiency is comparatively lower than Gd. Several modifications are being contributed for enhancing the contrast of Mn based nanoparticles to its successful application for MR imaging. Several modifications are being contributed for enhancing the contrast of Mn based nanoparticles to its successful application for MR imaging owing to its to its low toxicity and long electronic relaxation time of five unpaired electrons [150]. Niu et al reported Mn-based dual-mode contrast agents (DMCAs) based on the core-shell mesoporous silica spheres with Mn (Mn- DMSSs) for the simultaneous $\mathrm{T}_{1^{-}}$and $\mathrm{T}_{2}$-weighted MRI [151]. Mesoporous silica template was oxidised by $\mathrm{KMnO}_{4}$ leading to incorporation of Mn species, followed by calcination to eliminate the organic residue and finally the reduction of $\mathrm{Mn}$ ions to $\mathrm{Mn}^{2+}$ for improved MRI. The in vitro and in vivo MR imaging studies of Mn-DMSSs demonstrated exceptional contrast in $\mathrm{T}_{1-}$ and $\mathrm{T}_{2}$-weighted MR imaging. The authors suggest the high $r_{1}$ value of Mn-DMSSs was ascribed to three factors: a) very low concentration of $\mathrm{KMnO}_{4}(2 \mathrm{mM})$ to oxidize the CTAB molecules, b) generation of more $\mathrm{Mn}^{2+}$ ions through calcination that could increase $\mathrm{T}_{1}$-weighted MRI and c) mesoporous structure of Mn-DMSSs enhanced diffusion rate of water molecules inside the mesopores, thus increasing the $r_{1}$ relaxivity. On the other hand high $r_{2}$ value of Mn-DMSSs and enhanced $\mathrm{T}_{2}$ - weighted contrast effect could arise from ultra-small $\mathrm{Mn}$ oxide nanoclusters $(<2 \mathrm{~nm})$ in DMSSs resulting in the shortening of $\mathrm{T}_{2}$ relaxivity time. The authors advocate that Mn-loaded DMSSs could be employed as dual mode MR agents for diseases diagnosis in the future.

A novel mesoporous manganese silicate coated silica nanoparticle (MMSSN) for diagnostic and chemotherapeutic application was developed by Li et al. [152]. They adapted " $\mathrm{SiO}_{2}$ sacrifice and in situ silicate growth" strategy to develop the nanocarrier. The large surface area and exposed Mn paramagnetic centers to water molecules offered high longitudinal relaxivity. In addition, nanocarrier presented $\mathrm{pH} /$ redox-responsive $\mathrm{T}_{1}$-MRI feature in in vitro and in vivo conditions, when the nanoparticles was triggered in acidic conditions or presence of reducing agents. The efficacy of $\mathrm{pH}$-/redox-responsive MMSSN for in vivo tumour MR imaging was demonstrated in nude mice MDA-MB-231 cancer xenograft model. Substantial enhancement in positive signal was observed at tumour tissues over time that might be attributed to $\mathrm{pH}$ and GSH responsive characteristics of MMSSNs. Hu et al developed aptamer targeted $\quad \mathrm{Mn}_{3} \mathrm{O}_{4} @ \mathrm{SiO}_{2} \quad$ core-shell nanoparticles for targeted $\mathrm{T}_{1}$-MRI in human cervical 
carcinoma tumour-bearing mice [153]. Hydrophobic $\mathrm{Mn}_{3} \mathrm{O}_{4}$ core was encapsulated within an amino modified silica shell and rhodamine was doped into silica shell. The nanoprobe was further modified with amphiphilic PEG to improve biocompatibility and decorated with AS411 (aptamer) to the PEG chains as targeting ligands. Selective accumulation of nanoprobe in the in vivo tumour was demonstrated. $\mathrm{T}_{1}$-weighted $\mathrm{MR}$ images were acquired after intravenous injection of targeted nanoprobe to mouse model with tumour. A slight enhancement in contrast was observed in $T_{1}$ weighed MR images. After $12 \mathrm{~h}$ significant enhancement of contrast was observed indicating the accumulation of nanoprobe in the tumour. Signal enhancement was decreased after $24 \mathrm{~h}$, indicating either clearance or biodegradation of nanoprobe from mouse.

\section{Positron emission tomography}

Positron emission tomography (PET) offers biological information with exceptional sensitivity at molecular level and plays a significant role in molecular imaging [154]. Specific molecular probes labelled with positron-emitting radioisotopes are utilized for imagining in vivo biological processes [155]. In PET imaging three-dimensional images are acquired by detecting gamma rays that are emitted by a positron-emitting radionuclide or tracer that are conjugated to bioactive molecules. Owing to its extremely low background noise, high sensitivity and non-invasive imaging, PET is often used for evaluation of tumour biodistribution and pharmacokinetics studies. Commonly used PET radioisotopes for labelling drugs, targeting antibodies/peptides, and functional molecules include ${ }^{11} \mathrm{C},{ }^{18} \mathrm{~F},{ }^{15} \mathrm{O},{ }^{64} \mathrm{Cu},{ }^{68} \mathrm{Ga},{ }^{99} \mathrm{~m} \mathrm{Tc},{ }^{111} \mathrm{In}$, etc. General strategy to integrate PET imaging capability into nanocarriers is to attach a radio-metal via a metal chelator, such as 1,4,7,10-tetraazacy- clododecanetetraacetic acid (DOTA) or 1,4,7-triazacyclononane$\mathrm{N}, \mathrm{N}^{\prime}, \mathrm{N}^{\prime \prime}$-triacetic acid (NOTA). Most commonly used clinical PET-active isotope is ${ }^{18} \mathrm{~F} \quad\left({ }^{18} \mathrm{~F}\right.$-labeled fluorodeoxyglucose) to tracer high-glucose-utilising cells including brain, kidney and cancer cells. The short half-life of ${ }^{18} \mathrm{~F}$ and requirement of long circulation time in the body for specific uptake into target cells or organs makes ${ }^{18} \mathrm{~F}$ incompatible for the attachment of macromolecule-based targeted imaging probes (antibodies, peptides, etc) for in vivo PET study $[155,156]$. Owing to the short half-life of ${ }^{18} \mathrm{~F}$ (109.8 minutes) that leads to reduced time for observation, radionuclides with longer half-life $t_{1 / 2}$ with the combination of other imaging modalities in a single nanocarrier for long-term visualization in PET with a single administration at low particle dosage are being developed. Radiolabeled MSNs with ${ }^{124} \mathrm{I}\left(\mathrm{t}_{1 / 2}\right.$ $-100.2 \mathrm{~h})$ or ${ }^{64} \mathrm{Cu}\left(\mathrm{t}_{1 / 2}-12.7 \mathrm{~h}\right)$ have established the application for long- term PET imaging and their biodistribution studies [135, 157]. In most of the studies, complexing agents for PET radionuclides are positioned on exterior surface of mesoporous silica nanoparticles. Hence for long term biodistribution studies, mesoporous silica nanoparticles with the capability to load more number of PET active isotopes would be essential and the study can be performed with low concentrations of nanoparticle to acquire good data quality.

Short-lived radionuclides integrated in nanocarriers for example, ${ }^{18} \mathrm{~F}$ are often used to image within a short period of time after intravenous administration. However the major drawback is short half-life of the radionuclide. Kim et al introduced ${ }^{18} \mathrm{~F}$-labelled silica nanoparticle for PET imaging based on strain-promoted alkyne azide cycloaddition (SPAAC) conjugation of aza-dibenzocyclooctyne (DBCO) for in vivo imaging, thus demonstrating pretargeted PET imaging study by biorthogonal covalent ${ }^{18}$ F-labeling [158]. They first developed DBCO-based PEGylated MSNs (DBCO-PEG-MSNs). DBCO group functioned as an azide acceptor was introduced into PEG- amine moiety of PEGylated amino MSN to form DBCO-PEG-MSNs. To evaluate the SPAAC reaction rate and possibility of copper-free click reaction, DBCO-PEG- MSNs was reacted with ${ }^{18} \mathrm{~F}$ fluoropentaethylene glycolic azide under in vitro situations and confirmed the formation of ${ }^{18} \mathrm{~F}$-labeled azadibenzocyclooctatriazolic PEG-MSNs $\quad\left({ }^{18} \mathrm{~F}-\right.$ DBCOT-PEG-MSNs). Modified DBCO-PEG-MSNs nanoparticles were administrated via intravenously into mice with subcutaneous U87MG tumour. The radiotracer was injected after $24 \mathrm{~h}$ and corresponding PET images were developed. Another batch of mice were injected with only radiotracer. After $2 \mathrm{~h}$, PET images were compared between the two groups that displayed comparable uptake in all the tissues except in tumour. Pre-targeted mice exhibited considerably enhanced uptake in tumour than non-pre-targeted mice. Enhanced uptake in tumour was due to the formation of ${ }^{18} \mathrm{~F}-\mathrm{DBCOT}-\mathrm{PEG}-\mathrm{MSN}$ by SPAAC conjugation reaction in vivo. Pre-targeting method was extremely useful in drug delivery with MSNs for real-time monitoring by non-invasive imaging. Also this strategy could be adapted for successful PET imaging with a short half-life as a PET radionuclide $\left({ }^{18} \mathrm{~F}\right)$ along with other nanocomposite materials and targeting moieties that require a long bloodstream-circulation time. (Figure 12) 
Chen et al reported in vivo vascular imaging with multifunctional mesoporous silica nanoparticles incorporated with copper-64 $\left({ }^{64} \mathrm{Cu}, \mathrm{t}_{1 / 2}=12.7 \mathrm{~h}\right)$ and 800CW (NIRF dye) [159]. Vascular targeting of tumour angiogenesis was performed with TRC105 (a human/murine chimeric IgG1 monoclonal antibody) along with imaging modalities resulting in in vivo targeted PET/NIRF imaging of tumour vasculature. Tumour targeting efficacy, specificity and pharmacokinetics in 4T1 murine breast tumour-bearing mice was investigated through in vitro, in vivo, and ex vivo experiments. Accumulation of targeted ${ }^{64} \mathrm{Cu}-\mathrm{MSN}-800 \mathrm{CW}-\mathrm{TRC} 105(\mathrm{Fab})$ in the 4T1 tumour was clearly exhibited in PET imaging and was found to be $5.4 \pm 0.2 \% \mathrm{ID} / \mathrm{g}$ at $4 \mathrm{~h}$ post injection while the tumour uptake of non-targeted ${ }^{64} \mathrm{Cu}-\mathrm{MSN}-800 \mathrm{CW}$ nanoparticles in $4 \mathrm{~T} 1$ tumour was found to be less $(\sim 2$ $\% \mathrm{ID} / \mathrm{g}$ ) at all of the time points signifying the conjugation of targeting ligand [TRC105(Fab)] for enhanced tumour accumulation in vivo. The CD105 targeting specificity of nanocomposite, ${ }^{64} \mathrm{Cu}-\mathrm{MSN}-$ 800CW-TRC105 (Fab), was confirmed by blocking studies by administrating blocking dose (1 $\mathrm{mg} /$ mouse) of free targeting agent (TRC105) at $1 \mathrm{~h}$ before the injection of ${ }^{64} \mathrm{Cu}-\mathrm{MSN}-800 \mathrm{CW}-\mathrm{TRC} 105$. The results revealed significant reduction in tumour uptake to $2.3 \pm 0.2 \% \mathrm{ID} / \mathrm{g}$ at $4 \mathrm{~h}$ post injection indicating the potential of specific targeting of ${ }^{64} \mathrm{Cu}-\mathrm{MSN}-800 \mathrm{CW}-\mathrm{TRC} 105(\mathrm{Fab})$. The study reported successful vascular targeting and its $\sim 2$-fold enhancement of tumour accumulation of nanoparticles over passive targeting and as an attractive candidate for future cancer theragnostic application. The same researchers developed hollow mesoporous silica nanoparticle decorated with targeting moiety (anti-CD105 antibody), NIR dye (ZW800) and PET isotope ${ }^{64} \mathrm{Cu}$ ) for in vivo tumour targeted PET/NIRF imaging [129]. The hollow space of nanocarrier was loaded with Dox. In vivo PET imaging was performed in 4T1 murine breast tumour-bearing mice that expressed high level of CD105 on tumour neovasculature. In vivo biodistribution in mice with targeting, non-targeting and blocking groups were studied at different time points and accumulation of ${ }^{64} \mathrm{Cu}-\mathrm{HMSN}-\mathrm{ZW} 800-$ TRC105 was highest in 4T1 tumour than non-targeted group signifying the effect of targeted nanocarriers and potential of multimodal imaging. They also demonstrated PET to study time dependent uptake of CuS@ MSN in 4T1 tumour [160]. The uptake was increased and reached its highest concentration in tumour at $24 \mathrm{~h}$ post injection. Targeted nanoprobe, CuS@ MSN-TRC 105, exhibited highest uptake when compared to the CuS@MSN that in turn indicated the

(a)
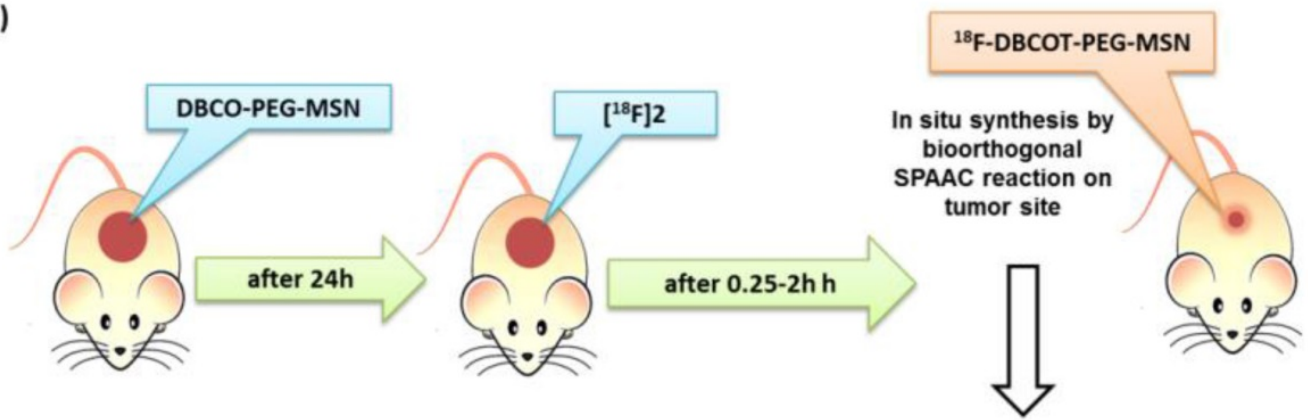

(b)

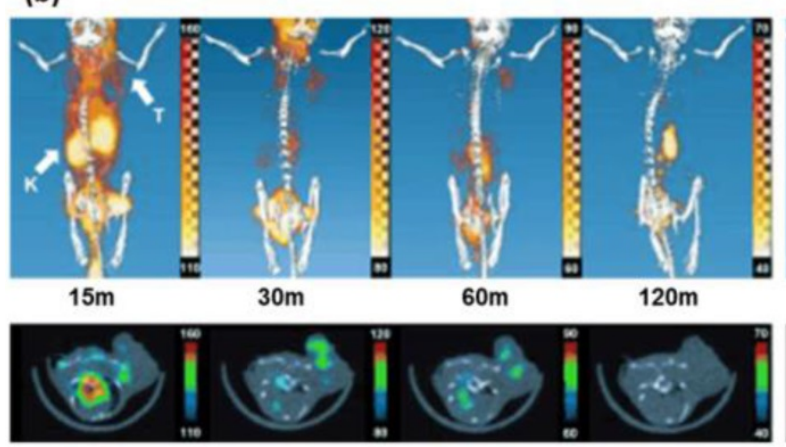

(c)

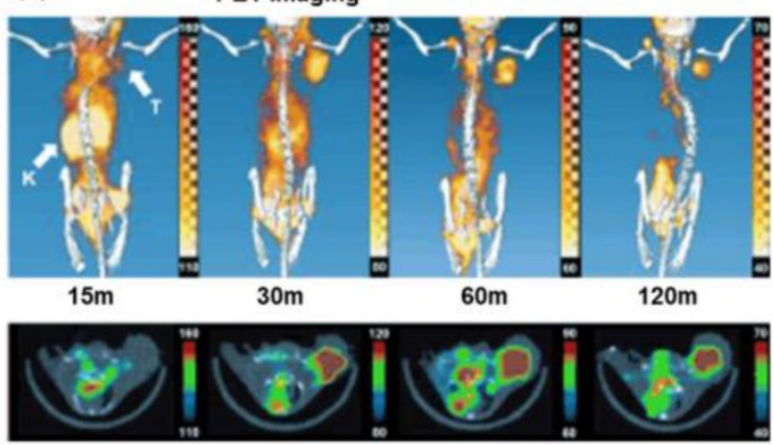

Figure 12: Pretargeting PET imaging study by bioorthogonal covalent $18 \mathrm{~F}$-labeling [171]. A) The procedure for the in situ synthesis of $18 \mathrm{~F}-\mathrm{DBCOT}$-PEG- MSNs in a living specimen by a bioorthogonal SPAAC reaction for the DBCO-PEG-MSN-pretargeting PET-imaging study. B),C) Three-dimensional reconstruction (upper) and transverse section (lower) combined PET-CT images of ${ }^{18 F}$-labeled azide $\left(\left[{ }^{18} \mathrm{~F}\right] 2 ; 2.6 \mathrm{MBq}\right.$ ) in a U87 MG tumor-bearing mouse given only [18F]2 alone (non-pretargeted; B) or a mouse given DBCO-PEG-MSNs $24 \mathrm{~h}$ earlier (pretargeted; C) recorded at 15, 30, 60, and 120 min after injection of [ $\left.{ }^{18 F}\right] 2$. $=$ kidneys. 
specificity of targeted nanoprobe for its biomedical application.

Multifunctional hollow mesoporous silica nanoparticles (HMSNs) for targeted tumour vasculature drug delivery and PET imaging was designed by Cai's group [46]. Amine functionalized HMSNs were conjugated to NOTA, further PEGylated and loaded with Sunitinib (anti-angiogenesis drug). Cyclo(Arg-Gly-Asp-D-TyrLys) (cRGDyK) peptide was employed as targeting ligand and radiolabeled with ${ }^{64} \mathrm{Cu}$ for PET imaging. The targeted nanoprobe $\left({ }^{64} \mathrm{Cu}-\mathrm{NOTA}-\mathrm{HMSN}-\mathrm{PEG}-\right.$ cRGDyK) displayed integrin specific uptake in in vitro and in vivo studies. Enhanced uptake of targeted nanoprobe $(\sim 8 \% \mathrm{ID} / \mathrm{g})$ was observed in the PET results signifying specificity of active targeting by nanoprobe in U87MG tumours and by passive targeting owing to EPR effect.

Miller et al designed mesoporous silica nanoparticles integrated with the stable and multiple ${ }^{89} \mathrm{Zr}$ isotope (half-life of 78.4 hours) ${ }^{89} \mathrm{Zr}^{4+}$ was attached to large-pore MSNs through covalent interaction of $\mathrm{p}$-isothiocyanatobenzyldesferrioxamine (DFO-NCS) that functioned as complexing agent [161]. ${ }^{89} \mathrm{Zr}^{4+}$ labelling was achieved within just a few minutes owing to high content of DFO on the MSNs ( $\left.{ }^{89} \mathrm{Zr}-\mathrm{DFO}-\mathrm{MSN}\right)$ ). The leaching of radionuclides from mesoporous silica nanoparticles was not observed from nanoprobe demonstrating the safety for imaging studies. Biodistribution studies was demonstrated in nude mouse model with prostate cancer tumour and high signal strength was observed in PET imaging with ${ }^{89} \mathrm{Zr}$-DFO-MSNs in liver, spleen and lungs. Biodistribution of radiolabeled ${ }^{89} \mathrm{Zr}^{4+}$ as a salt solution in mice was homogenous all over the body and it was largely present in the blood. High-quality in vivo PET images were acquired at mesoporous silica nanoparticles concentrations about an order of magnitude lower than previously reported PET-active mesoporous silica nanoparticles. However there was no prominent accumulation of ${ }^{89} \mathrm{Zr}$-DFO-MSNs in tumour

\section{$\mathrm{X}$ ray Computed tomography ( $\mathrm{X}$ ray- $\mathrm{CT})$}

Computed tomography (CT) is one of the commonly used diagnostic tools in most hospitals due to its high spatial resolution, efficiency, availability, and low cost compared to other diagnostic instrumentation. Currently used contrast agents are iodine-containing molecules, and are generally used for angiography and urography. The short resident time of iodinated compounds in internal organs for acquiring steady images and adverse allergic effects of iodinated contrast agents in allergic patients demands the need for new contrast agents. Ideally for a compound to be a CT contrast agent, it should possess high $\mathrm{X}$-ray absorption coefficient. CT imaging capacity is determined by $\mathrm{X}$-ray absorption coefficient which in turn is dependent on atomic number $(Z)$. Metalic nanoparticles based on $\mathrm{Au}(Z=79)-$, $\mathrm{Bi}(\mathrm{Z}=$ 83)-, Ta $(Z=73)-$, and $\mathrm{Yb}(Z=70)$ have higher atomic number and larger $X$-ray absorption coefficients than conventional iodinated contrast agents $(Z=53)$ and are potential alternatives for iodinated contrast agents in future CT imaging [162-165]. Gold nanoparticles have been extensively characterized owing to its strong X-ray absorption for gaining $X$-ray images with high contrast. Advanced research on X-ray imaging with metallic $\mathrm{Au}$ nanoparticles has enabled the technique to image tissues at nanometer level [166, 167]. The residence time of $\mathrm{Au}$ nanoparticles in internal organs is extended due to its larger size than that of iodinated molecules, which may ultimately allow steady imaging.

Toxicity associated with metallic nanoparticles is considered to be a major concern for the application as effective contrast agents for CT [168, 169]. Successful encapsulation, entrapment or adsorption of metallic nanoparticles in a biocompatible nanocarrier is an effective method for reducing inherent toxicity. The development of core shell nanoparticle where metallic nanoparticles form core and biocompatible shell protects the contrast agent and reduce the toxicity. Mesoporous silica is the ideal material for the shell owing to its inert nature and biocompatibility. There are several reports on core-shell particles composed of $\mathrm{Au}$ nanoparticle as core in different morphology including spheres, rods, cages, etc. and mesoporous silica shell [170, 171].

Hayashi et al developed NIR fluorescent silica-coated gold nanoparticle clusters and demonstrated dual modal imaging (NIR fluorescence and CT) for visualization of lymph nodes and lymph vessels [136]. In cancer patients, lymph nodes are often removed to prevent cancer metastases and lymph nodes and lymph vessels are small, not easily detectable and its removal is complicated. NIR fluorescent silica-coated gold nanoparticle clusters $\left(\mathrm{Au} @ \mathrm{SiO}_{2}\right)$ with a high X-ray absorption coefficient were developed and demonstrated the accumulation of nanoparticles in lymph nodes through lymph vessels. CT imaging precisely located tumour and determined the size of lymph nodes and lymph vessels without dissection. (Figure 13) 
A core-shell structured theragnostic mesoporous silica based nanoplatform functionalized by components including photosensitizer Ce6, carbon dots (CDs), and dual modal imaging agent Gd (III) ions for MRI and CT has been designed by Yang et al [147]. Thermo/pH-sensitive polymer poly[(N-isopropylacrylamide)-co-(methacrylic acid)] (P(NIPAm-coMAA)) was used to encapsulate pores and outer shell of core-shell nanospheres. Gadolinium (Gd) nuclide is reported to function both as an MRI and CT contrast agent. A sharp signal enhancement was observed and CT Hounsfield-unit values (HU) value was enhanced linearly with the increase in the concentration of DOX-GSCCP concentration. For in vivo effectiveness the mouse was injected with nanoparticles and X-ray

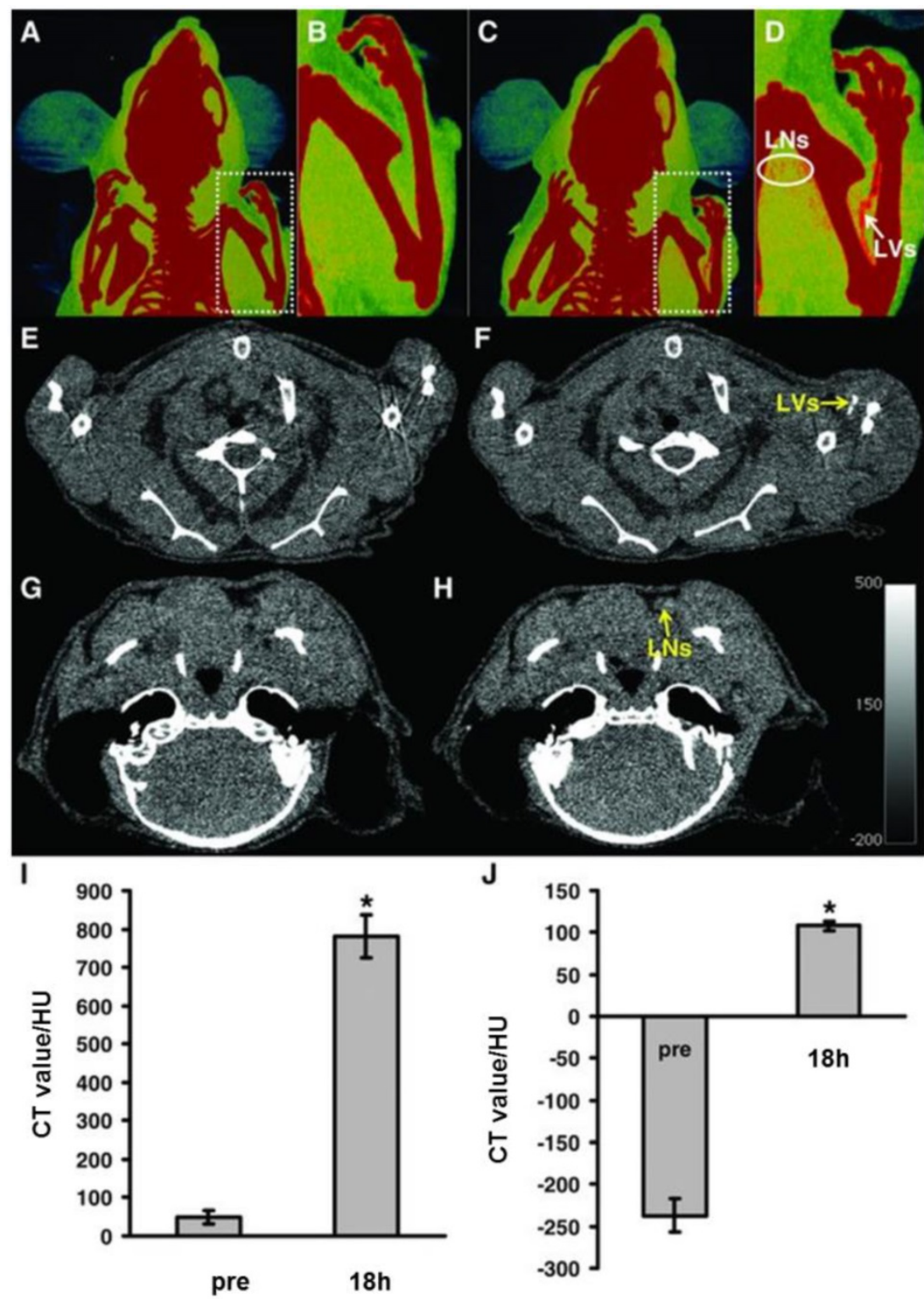

Figure 13: $C T$ MIP images of a mouse injected with $\mathrm{Au} @ \mathrm{SiO}_{2}$ nanoparticles intradermally into the left paw: A) pre-injection, B) close-up image of the region enclosed by a square in A, C) $18 \mathrm{~h}$ post-injection, and D) close-up image of the region enclosed by a square in C. Axial CT images of the mouse: $E, G$ ) pre-injection and F,H) $18 \mathrm{~h}$ after injection. Average CT values differences of I) LVs and J) cervical LNs between pre-injection and $18 \mathrm{~h}$ after injection [149].
CT imaging was performed. Accumulation of nanoparticles was increased at tumour site after injection and pharmacokinetic profile revealed CT single enhancement in liver owing to the presence of $\mathrm{Gd}$ ions in nanoparticles from blood to the liver. The in vitro and in vivo $\mathrm{CT}$ results demonstrate that DOXGSCCP nanoparticles function as a contrast reagent for $\mathrm{CT}$ imaging.

$\mathrm{Au}$ nanostar (NS)-coated hollow mesoporous silica nanocapsule (HMS) encapsulated with perfluorohexane (PFH) was developed by $\mathrm{Li}$ and co-workers [172]. The nanoconstruct was then surface modified with poly(ethylene glycol) (PEG) and studied its multimodal tumour imaging potential by ultrasonic (US)/computed tomography (CT)/photoacoustic (PA)/thermal imaging. Multifunctional HMSs@AuPFH-mPEG NSs (HAPP) was stable in colloidal condition and was cytocombatible. For in vivo CT imaging, HAPP was injected into mouse via tail vein. CT images of tumours collected at different time points after injection was computed to determine $\mathrm{CT}$ value of tumours. The peak CT values before and after was compared and tumour region presented a peak CT value of $51.8 \mathrm{HU}$ after $2.0 \mathrm{~h}$ postinjection that was 1.6 times higher than before injection (32.8 HU).

$\mathrm{Au}$ nanospheres coated with indocyanine green (ICG)-loaded mesoporous silica nanoparticles (Au@MSNs-ICG) was developed to locate the tumour site, diagnosis by NIR and CT bimodal imaging [173]. The combined NIR/CT imaging accurately positioned tumour for its extended PTT treatment. CT imaging of Au@MSNs-ICG diffused in PBS at different concentrations exhibited different $C T$ values and $C T$ HU values were linear with increasing concentration. X-ray attenuation of $\mathrm{Au} @ \mathrm{MSN}$-ICG was superior to that of commercially used iopromide. 3D biodistribution of NP was performed by in vivo tomographic imaging of liver xenograft mouse model with fluorescent molecular tomography (FMT) and CT. CT imaging capability of Au@MSNs- ICG was also demonstrated in in vivo tumour bearing mice. Significant CT contrast 
signals in tumours were observed after $3 \mathrm{~h}$ post injection of NPs. After $6 \mathrm{~h}$ post injection, entire tumour area displayed higher signal intensity suggesting higher accumulation of Au@MSNs-ICG.

Song et al developed $\mathrm{Au} @ \mathrm{mSiO}_{2}$-TTA/IR783/PVP by coating Au nanospheres with positively charged $\mathrm{mSiO}_{2}$ by co-hydrolysis of $\mathrm{N}$-trimethoxysilylpropyl-N,N,N-trimethylammonium chloride (TTA) and tetraethoxysilane (TEOS) [130]. Positively charged $\mathrm{mSiO}_{2}$ retained mesoporous structure for the adsorption of IR-783 into mesoporous structure through electrostatic interaction. The nanoprobe was further modified with polyvinylpyrrolidone (PVP) to be utilised for in vitro and in vivo bioimaging by $\mathrm{CT}$ and fluorescence imaging. (figure 14) $\mathrm{Au} @ \mathrm{mSiO}_{2}-\mathrm{TTA}$ nanoprobe with sufficient thick to confirm adequate loading of IR-783 and to avoid quenching of IR-783 from the $\mathrm{Au}$ nuclear core was designed. X-ray $\mathrm{CT}$ imaging potential of nanoprobe was studied in male nude mice through tail vein injection and distribution of nanoprobe in spleen and liver was observed in CT imaging. CT image of spleen was higher than liver and the researchers speculate that spleen might have accumulated more probe than liver signifying selective accumulation of nanoprobe in different organs.

$\mathrm{WS}_{2}$ nanosheets developed by Yang et al, functioned as core that exhibited strong NIR and X-ray absorbance that was exploited for photothermal therapy and X-ray CT imaging, respectively [145]. Upon laser irradiation, photothermal effect of $W^{2}$ stimulated Dox release from mesoporous silica shell of $\mathrm{WS}_{2}$-IO@MS- PEG, resulting in enhanced cancer cell toxicity. $\mathrm{WS}_{2}$ absorb X-ray and offered enhanced contrast in CT imaging. CT images of $\mathrm{WS}_{2}$-IO@MS-PEG solutions demonstrated linear increase of $\mathrm{HU}$ values upon increased concentrations of $\mathrm{WS}_{2}$-IO@MS-PEG. Enhanced CT contrast was observed in the tumour at $24 \mathrm{~h}$ post injection in $4 \mathrm{~T} 1$ tumour-bearing Balb/c mice after intravenous administration of $\mathrm{WS}_{2}$-IO@MS-PEG.

\section{Ultrasound imaging}

Among various molecular imaging modalities, ultrasound has received significant attention in clinical fields owing to its non-invasive and real-time imaging with enhanced spatial resolution of a lesion with the design of complex ultrasound contrast agents (UCA) [174]. Recently, in addition to diagnostic features, high intensity focused ultrasound (HIFU) with frequencies in the range of $0.8-3.5 \mathrm{MHz}$ has therapeutic potential and mediates ultrasound activated drug delivery to annihilate tumour cells. Design of echogenic nanoparticles that serve as ultrasound contrast enhancer and ultrasound responsive drug delivery are extremely efficient for theragnostic applications. Echogenic particles for imparting contrast are denoted as microbubbles are made up of gaseous inner core [air or bioinert heavy gases such as perfluorocarbons (PFCs), sulphur hexafluoride and nitrogen] stabilized by different shell materials including proteins, lipids or polymers. Conventional micro-sized echogenic particles have disadvantages including large particle, short half-life for circulation, and inability for tissue specific targeting [175]. For in vivo real-time imaging echogenic particles with long-term stability along with favourable physicochemical properties should

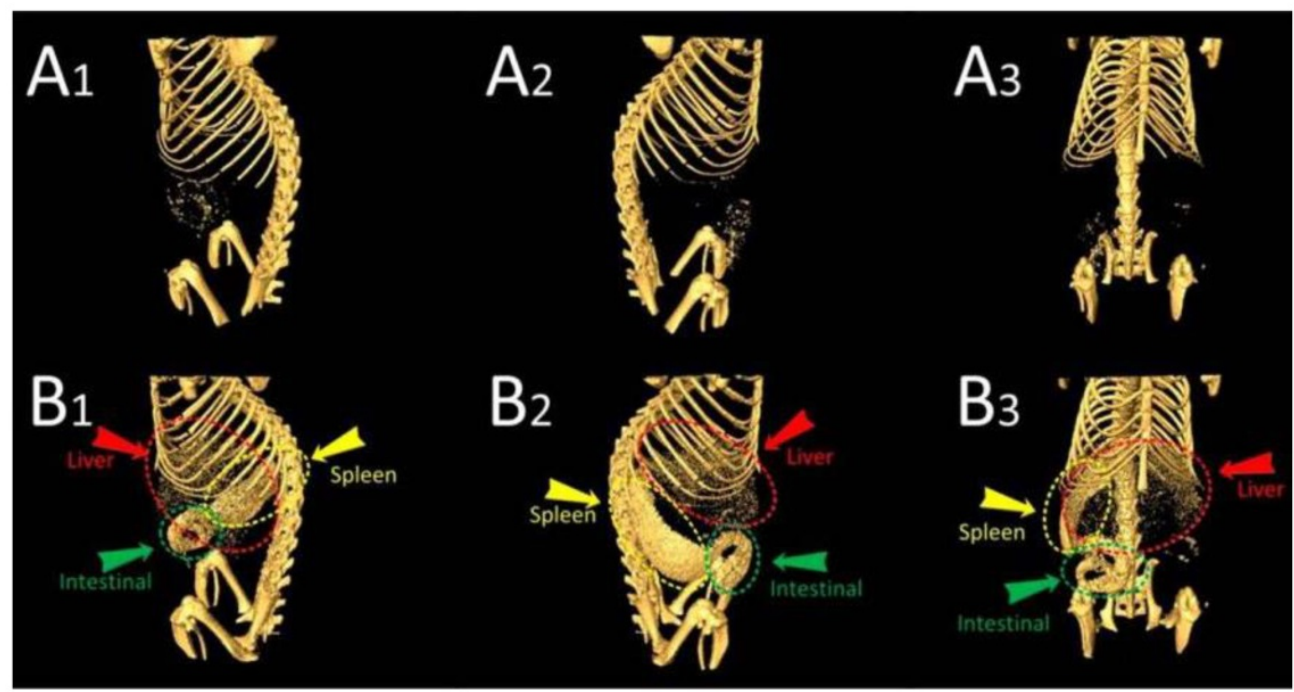

Figure 14: (i) TEM of $A u @ m \mathrm{miO}_{2}-$ TTA/IR-783/PVP under different magnifications $\left(\mathrm{A}_{1}, \mathrm{~A}_{2}, \mathrm{~B}_{1}, \mathrm{~B}_{2}\right)$; (ii) (A) CT imaging of different concentrations of the $\mathrm{Au} @ \mathrm{mSiO}_{2}-\mathrm{TTA} / \mathrm{IR}-783 / \mathrm{PVP}$ probe; (B) CT imaging of the same concentrations of Au@mSiO $\mathrm{m}_{2}-\mathrm{TTA}, \mathrm{Au} @ \mathrm{mSiO}_{2}-\mathrm{TTA} / \mathrm{IR}-783$, and Au@mSiO 2 -TTA/IR-783/PVP, respectively and (iii) CT images of nude mice (A) before and (B) $4 \mathrm{~h}$ after injection from different views $(1-3)$ [143]. 
be assured. Echogenic nanoparticles could demonstrate whole-body distribution and efficient extravasation; however, they are incapable for generating enough echo signals to image the lesion. The use of gases for generating contrast also makes it difficult for developing distinct types of echogenic particles holding physical and echogenic properties for efficient ultrasound imaging. Mesoporous silica nanoparticles are inorganic, solid, and highly porous nanostructures that have been subjected for application as a long-lasting in situ UCA [176, 177]. Recent studies have successfully exploited silica nanoparticles as contrast enhancing agents for ultrasound imaging at conventional diagnostic frequencies. Mesoporous silica nanoparticles are found to be more effective in static systems, such as local tumour environments, where they accumulate in a layer to effective imaging.

Chen et al designed a novel type of silica nanoparticle with a concave cup shaped mesoporous structure that had the capability to enhance ultrasound signal [178]. The exosome-like silica (ELS) nanoparticles had substantial ultrasound impedance mismatch for regenerative medicine imaging. ELS was fabricated via an emulsion soft-template method and ELS nanoparticles were formed with the co-condensation of bis(triethoxysilyl)ethane (BTSE) and $40 \%$ bis(3-tri- methoxysilyl-propyl)amine (TSPA) with positively charged amine groups embedded in the framework that was suitable for labelling mesenchymal stem cells. ELS exhibited the strongest echogenicity under in vitro agarose phantom at 40 $\mathrm{MHz}$ that permitted a lower nanoparticle dose to generate the same ultrasound contrast. The echogenicity of ELS-labelled human mesenchymal stem cells was increased than unlabelled cells and cells labelled with other silica nanoparticles [Stober silica nanoparticles (SSN), MCM-41 mesoporous silica nanoparticles (MSN), and mesocellular foam silica nanoparticles (MCF)] owing to its positive charge that facilitated cellular uptake. The in vivo ultrasound images of subcutaneously injected nude mice exhibited substantial increase of echogenicity of transplanted ELS-labelled stem cells than unlabelled cells.

Yildirim et al studied the properties of nanoparticle on stabilization of nanobubbles and nucleation of acoustic cavitation by nanobubbles [179]. They investigated mesoporous silica nanoparticles of $100 \mathrm{~nm}$ size with different morphologies to study the effects of textural parameters, hydrophobicity, and hydrophilic surface modification on acoustic cavitation by porous nanoparticles. Presence of carbon impurities during synthetic steps or hydrophobic modification with methyl groups was essential for stabilization of nanobubble. They demonstrated acoustic cavitation was mainly nucleated by nanobubbles stabilized at the surface of mesoporous nanoparticle and not inside mesopores. The hydrophobic and rougher surfaces of mesoporous silica nanoparticles initiated acoustic cavitation. Nanoparticle with rough surface was essential to preserve surface nanobubbles after coating the surface with hydrophilic macromolecules for in vivo applications. They also developed per-fluorocarbon-free, air encapsulated, mesoporous silica with hydrophobic interior based nanoscale ultrasound contrast agent [180]. Mesoporous silica contrast agent was of $100 \mathrm{~nm}$ in size with $3 \mathrm{~nm}$ pore size. The stabilized nanoscale air pockets nucleateses: bubbles by inertial cavitation under an ultrasound pulse. Cavitating bubbles from nanoparticles then scatter and radiate sound that was perceived by ultrasound imaging transducer. When exposed to sispiHIFU, nanoprobe generated ultrasound signal at lower dosage $\left(10^{10}\right.$ particles $\left.\mathrm{mL}^{-1}\right)$ with continuous imaging at least for $20 \mathrm{~min}$. Ultrasound response of mesoporous and nonporous silica nanoparticles with different surface chemistry and various conditions was studied, that eventually supported the hypothesis where entrapped air in nanoprobe could trigger the formation of bubble nuclei that could act as acoustic scatterers. The nanoprobe was biocompatible with very little hemolysis in whole blood, and cavitation activity on red blood cells was investigated for blood pool imaging. Further large-scale production of air filled contrast agents and storage either in PBS or lyophilization was also demonstrated.

In another study, a typical hard-templating method was adapted to synthesize organic/inorganic hybrid hollow periodic mesoporous organosilicas (HPMOs) as contrast-enhanced ultrasound-imaging agents and HIFU triggered drug release [181]. Core/shell structured $\mathrm{SiO}_{2} @ \mathrm{PMOs}$ were prepared by coating a PMOs shell onto the surface of silica NPs using 1,4-bis(triethoxysilyl)benzene (BTEB) as organosilica precursors and silica core as etched away by alkaline etching. HPMOs presented enhanced contrast in ultrasonography both under in vitro and in vivo models. In vitro studies indicated that contrast enhancement generated by HPMOs was stable under high mechanical indexes in the range from 0.6 to 1.2 than commercial SonoVue, which could image only under low mechanical indexes of contrast mode. Under in vivo studies after administration of HPMOs, tumour tissues exhibited noticeable contrast enhancement in ultrasonography image owing to rapid diffusion into tumour tissue. HPMOs also demonstrated high in vivo stability and retained 
contrast even after 7 days than commercial clinically used SonoVue. The study suggests that HPMOs can be used for the real-time ultrasound imaging to determine the progression of lesion tissues.

Jin et al developed superhydrophobic mesoprous silica nanoparticles as ultrasound contrast agents [182]. Interfacial nanobubbles (INBs) can nucleate bubbles formation upon ultrasound exposure and it can nucleate cavitation hundreds of times thus improving sonochemical efficiency. In addition, INBs on a hydrophobic surface have an extensive lifetime in the orders of days than the bulk nanobubbles with lifetime in the orders of microseconds. Based on this concept, mesoporous silica nanoparticles with large surface areas with different levels of hydrophobicity with encapsulated air were used as contrast agents. The bubbles developed from super hydrophobic mesoporous silica nanoparticles was persistent at least $30 \mathrm{~min}$ at a mechanical index of 1.0. In vitro B-mode contrast intensity studies revealed that F- mesoporous silica nanoparticles (super hydrophobic and porous) exhibited strong contrast intensity than F-SS (super hydrophobic and nonporous), while mesoporous silica nanoparticles displayed insignificant contrast. The super hydrophobic mesoporous silica nanoparticles could function as promising theragnostic agents responsive to ultrasound stimulation. Martinez et al synthesized perfluoropentane filled hollow silica and silica boron microshells for colour Doppler US imaging (CDI) in human breast tissue [183]. Perfluorocarbon gas-filled silica capsule injected into the tissue persisted for several days that were used to acquire 3D images of human breast tissues. They reported on perfluoropentane gas filled iron-silica nanoshells for colour Doppler ultrasound to guide surgical resection [184]. The function of iron silica nanoshells as ultrasound guide marker for tumour resection was studied in Py8119 tumour bearing mice and was imaged by colour Doppler ultrasound over the course of 10 days. Zhang et al reported double-scattering/reflection in a single nanoparticle for enhanced ultrasound imaging by using rattle-type mesoporous silica nanostructure based on hydrophobic shell-protected selective etching [185]. Acoustic properties of rattle structure were compared with solid and hollow silica NPs. The additional interface by designing an intermediate core in a hollow NP enhanced US imaging capability by improving scattering and reflection effects. The scattering contribution ratios of rattle-type mesoporous silica nanoparticle to solid- $\mathrm{SiO}_{2} /$ hollow- $\mathrm{SiO}_{2}$ and rattle-type mesoporous silica nanoparticle to hollow mesoporous silica nanoparticle was 1.58 and 1.51, respectively signifying double backscattering in a single rattle-type mesoporous silica nanoparticle. Linear scattering contribution of rattle-type mesoporous silica nanoparticle was quantified to be $44 \%$ and $47 \%$ higher than solid and hollow structure, respectively. Rattle-type mesoporous silica nanoparticle performed better in in vitro and in vivo ultrasound imaging than solid and hollow nanostructure owing to the characteristic double-scattering/reflection interfaces. In vitro and in vivo US imaging was confirmed in L929 cell and grafted VX2 solid liver tumour in rabbits, respectively and results established multilayer-structured design effectively improved US imaging of ultrasound contrast agents based on the "double-scattering/reflection" mechanism.

\section{Raman Imaging}

Raman imaging based optical imaging depends on inelastic interaction of light with matter. Raman reporters (molecules) generate unique and distinct fingerprint-like Raman spectra. Raman Effect is basically weak; however Raman cross-section of Raman reporters can be significantly enhanced when they are near to metal nanoparticle surfaces through SERS [186]. Nanoprobes based on SERS offer excellent specificity and sensitivity for cancer imaging. Aptamer (Mucin1) targeted SERS nanoprobe consisting of AuNP core, IR dye coating, and silica shell for breast cancer was developed and used for specific imaging [187]. The silica coating offered protective coating to the nanoprobe. Although SERS based nanoprobes were promising, the signal strength varied depending on accumulation of nanoparticles in different tumour types. Raman nanoparticles with enhanced signal intensity for attaining low limits of detection was developed for visualization of malignant tumors and premalignant lesions through microscopically in mice models. Surface-enhanced resonance Raman scattering (SERRS) nanoprobes resonant in NIR window was developed. Raman reporter molecules resonant in NIR window with the detection laser $(785 \mathrm{~nm})$ was incorporated with a metal core exhibiting localized surface plasmon resonance in NIR window. The imaging probe was encapsulated in biocompatible silica layer for efficient loading of resonant Raman reporter at the metallic core surface generating a core shell nanoprobe that was superior to previously developed non-resonant SERS nanoprobes [188].

SERRS-nanoprobe comprising of Raman reporter resonant in the NIR window and star-shaped gold core was developed via primer-free silication method [189]. Silica coated SERRS-nanostars facilitated detection of macroscopic malignant lesions 
in mouse models of pancreatic cancer, breast cancer, prostate cancer, and sarcoma without targeting moiety. Premalignant lesions in pancreatic and prostatic neoplasias were detected with nanoprobes. Dual modal imaging based on SERRS offers superior potential for non-invasive imaging with enhanced sensitivity and spatial resolution. Radiolabeled SERRS nanoparticles coated in silica shell for PET-SERRS tumor imaging was developed based on chelator free approach. PET-SERRS nanoparticles were demonstrated for including lymph node (LN) tracking, intraoperative guidance for LN resection, and cancer imaging after intravenous injection. PET-SERRS nanoprobe coud be tuned for whole-body imaging as a pre-operative guide and for image guided surgery. Another demonstrated combination of optical and optoacoustic imaging method for intraoperative brain tumor was investigated by Volker et al [190]. Gold nanostar, Raman reporter, and silication was employed to generate dual-modality contrast agents for surface enhanced resonance Raman scattering (SERRS) and multispectral optoacoustic tomography (MSOT) for imaging glioma margins in glioblastoma (GBM) patients. MSOT signal correlated with SERRS images and MSOT captured real time, deeply seated GBMs in 3D with superior specificity. The nanoprobe could be potential tool for image-guided resection of brain tumors.

\section{Biocompatibility and pharmacokinetic properties}

Mesoporous silica nanoparticles are classified as "Generally Recognized as Safe" by FDA owing to their in vivo biocompatible property. Silica particles degrade to water-soluble orthosilicic acid that is absorbed to form silica as trace element. Several in vitro studies and in vivo studies support biocompatibility of mesoporous silica nanoparticles [191-194]. For biomedical applications, the biological fate of nanoparticles is greatly dependent on size, charge, chemical composition, aggregation and surface chemistry. There are several studies investigating the physicochemical behaviour, biological fate with different surface modifications. Pharmacokinetics profile of drug loaded nanoparticles is associated with several factors including biocompatibility, cytotoxicity, efficacy of delivery, etc. and hence investigation of pharmacokinetic profile of mesoporous nanostructures as drug delivery system in biodistribution, retention, circulation, release and biodegradation are garnering attention. Mesoporous silica nanostructures permits sufficient control over pharmacokinetic property of loaded dug molecule than administration of free drug. When a free drug is administered either orally or intravenously concentration of drug in serum will be very high which then reduces till next dose. The high concentration could reach to toxic level and low concentration could lead to sub optimal levels. Nanoparticles offers controlled slow and sustained drug delivery which maintains an optimum concentration of drug for period of time. In addition to drug delivery, nanoparticles protect drug in the nano environment and permit loading of multiple drugs. However, systemically administered nanoparticles need to circulate in blood as long as possible to provide sufficient dose of therapeutics to target tissues. Approaches to enhance circulation time by coating with inert polymer (eg: PEG) over core shell mesoporous silica nanoparticles that resists interactions with blood stream components rendering 'stealth' properties are being commonly used. Researchers demonstrated that higher molecular weight PEG (from 10 to $40 \mathrm{kDa}$ ) and smaller diameter templates led to lower level of phagocytic blood cell association of PEGylated mesoporous silica NPs [195]. Theragnostic core-shell magnetic mesoporous silica nanoparticles coated with polyethylene glycol (PEG) or 1,2-dimyristoyl-sn-glycero-3-phosphocholine (DMPC) lipid bilayer was developed and influence of surface composition on dispersion, protein binding and cell membrane interaction was studied [196]. The coating offered excellent biocompatibility and influenced their biodistribution. Nanoparticles coated with DMPC displayed accumulation in liver and less accumulation in lung when compared with PEG and bare nanoparticles. SEERS-MSOT nanosystem was developed and the pharmacokinetic profile of nanostars was determined by MSOT and validated using SERRS imaging [190]. SERRS-MSOTnanoparticles were accumulated in cortex, glioblastoma and basal ganglia 5 min after injection. After $3 \mathrm{~h}$ postinjection, SERRS signal was detected within tumour area and in small vessels in subarachnoid space along sulci demonstrated slow clearance of nanoparticles from circulation and accumulation within the glioblastoma. SERRS-MSOT nanostars extravasated into perivascular niches of vascularized tumor and presented enhanced retention owing to their $\approx 100 \mathrm{~nm}$ size and retained within the tumour. Biodistribution of upconversion/magnetic silica-coated $\mathrm{NaGdF}_{4}: \mathrm{Yb}^{3+} / \mathrm{Er}^{3+} @ \mathrm{SiO}_{2}$ particles demonstrated that the nanoparticles are non-toxic at moderate concentrations and particle localization within the organs displayed vascular localization [197]. Thus mesoporous silica structure offer superior biocompatibility, drug loading potential and an ideal paradigm for surface modifications. PEGylation facilitates escape from mononuclear phagocytic 
system by enhancing the circulation time and longer residence time. Although biocompatibility, biodegradability and pharmacokinetics properties of Mesoporous silica nanoparticles are critical elements for future applications, concerns over acute and chronic toxicity, teratogenic toxicity, genotoxicity, long-term in vivo studies, pharmacodynamic properties of nanoconjugates still needs to be addressed. Therefore, more studies referring to elements could significantly favour the successful application of these versatile nanoconjugates for clinical applications.

\section{Conclusion and future prospective}

The development in nanoscience and nanotechnology has gifted excellent opportunity in design and development of innovative C/S-MSN with multifunctional capabilities for biomedical applications. Multifunctional C/S-MSN harness multiple imaging modalities and therapeutic entities as promising tool in the treatment and diagnosis of cancer. C/S-MSNs as theragnostic nanoplatform offer effective targeted therapy, enhanced drug delivery and controlled release, multiple therapy and real-time monitoring facilitated with contrast agents. Application of biocompatible C/S-MSNs with cores of different functionalities and mesoporous shell is widely explored for targeted chemotherapy, radiotherapy, phototherapy, magnetic hyperthermia, immunotherapy and gene therapy. Stimuli-responsive controlled drug release from C/S-MSNs have demonstrated enhanced therapeutic efficacy with reduced side effects. C/S-MSNs also established as efficient imaging in in vitro and in vivo tumor imaging that can be further extrapolated for clinical applications. MRI, fluorescence imaging and $\mathrm{X}$-ray CT are main imaging strategies that are widely studied with $\mathrm{C} / \mathrm{S}-\mathrm{MSN}$ s. Imaging of tumor with fluorescent moieties protected in C/S-MSNs have been successfully demonstrated in in vitro and in vivo models, however it experiences several issues to be used in real body imaging. Alternatively, NIR dyes as contrast agents in C/S-MSN reduce background fluorescence, which can be used as an attractive nanoplatform for deep tissue imaging.

Although there have been progress and promises, nanotheragnostic platform still demand significant improvement to be used for clinical application. While designing nanotheragnostics, the safety, stability and complexity of nanoparticles have to be emphasized. A comprehensive assessment of toxicity including cytotoxicity (acute and long term), immunotoxicity and genotoxicity of C/S-MSNs has to be addressed extensively. In addition, pharmacokinetics and biodistribution, has to be evaluated significantly. Although there are several reports on biocompatibility of C/S-MSNs the final verdicts on cytofriendly nature is still in debate. Extensive research is still needed to conclude their safe use in biosystem by investigating in small animals and primates. Currently targeted nanoparticles are given significant consideration, however accumulation of these nanostructures inside liver and spleen is prominent that might develop unwanted side effects. Therefore the design of nanoparticles with excellent targeting capability has to be improved. Combination of multiple therapies for efficient treatment of cancer with C/S-MSN can be developed to target multiple features of cancer including drug resistances in cancer. In addition, design of nanomedicine with multiple therapies should be simple and not demand higher level of complexity. The ultimate goal on development of multifunctional C/S-MSNs to attain all the desired functionalities in an all-in-one nanosystem with complete features to detect and kill cancer cells has to be given prime importance.

More promising developments in tumor treatment and diagnosis with C/S-MSNs are required for its successful application in biomedical field. Collaborative efforts by combining the expertise and knowledge from multidisciplinary fields including nanotechnology, cancer biology, and pharmaceutics is extremely vital for the development and successful application of theragnostic nanoparticles in clinics and as predictive and personalized medicine by effective translational research.

\section{Acknowledgement}

The author (ARG) thanks University of South Australia for providing financial support.

\section{Competing Interests}

The authors have declared that no competing interest exists.

\section{References}

1. Rosenholm JM, Sahlgren C, Lindén M. Towards multifunctional, targeted drug delivery systems using mesoporous silica nanoparticles opportunities \& challenges. Nanoscale. 2010; 2: 1870-3.

2. Coll C, Mondragón L, Martínez-Máñez R, et al. Enzyme-mediated controlled release systems by anchoring peptide sequences on mesoporous silica supports. Angew Chem Int Ed. 2011; 50: 2138-40.

3. Lai $\mathrm{CH}$, Lai NC, Chuang YJ, et al. Trivalent galactosyl-functionalized mesoporous silica nanoparticles as a target-specific delivery system for boron neutron capture therapy. Nanoscale. 2013; 5: 9412-8.

4. Tang F, Li L, Chen D. Mesoporous silica nanoparticles: Synthesis, biocompatibility and drug delivery. Adv Mater. 2012; 24: 1504-34.

5. Lou XW, Archer LA, Yang Z. Hollow micro/nanostructures: Synthesis and applications. Adv Mater. 2008; 20: 3987-4019.

6. Wan Y, Zhao D. On the controllable soft-templating approach to mesoporous silicates. Chem. Rev. 2007; 107: 2821-60.

7. Lee H, Char K. Morphological changes from silica tubules to hollow spheres controlled by the intermolecular interactions within block copolymer micelle templates. ACS Appl Mater Interfaces. 2009; 1: 913-20. 
8. Mandal M, Kruk M. Family of single-micelle templated organosilica hol low nanospheres and nanotubes synthesized through adjustment of organo silica surfactant ratio. Chem Mater. 2012; 24: 123-32.

9. Bao Y, Wang T, Kang Q, Shi C, Ma J. Micelle template synthesis of hollow silica spheres for improving water vapor permeability of waterborne polyurethane membrane. Sci Rep. 2017; 7: 46638

10. Yeh YQ, Chen BC, Lin HP, et al. Synthesis of hollow silica spheres with mesostructured shell using cationic-anionic-neutral block copolymer ternary surfactants. Langmuir. 2006; 22: 6-9.

11. Tan B, Lehmler H, Vyas SM, et al. Fluorinated-surfactant-templated synthesis of hollow silica particles with a single layer of mesopores in their shells. Adv Mater. 2005; 17: 2368-71.

12. Li J, Liu J, Wang D, et al. Interfacially controlled synthesis of hollow mesoporous silica spheres with radially oriented pore structures. Langmuir. 2010; 26 : 12267-72.

13. Li CY, Ma C, Wang F, et al. Preparation and biomedical applications of core -shell silica/magnetic nanoparticle composites. J Nanosci Nanotechnol 2012; 12: 2964-72.

14. Blas H, Save M, Pasetto P, et al. Elaboration of Monodisperse Spherical Hollow Particles with Ordered Mesoporous Silica Shells via Dual Latex/Surfactant Templating: Radial orientation of mesopore channels. Langmuir. 2008; 24: 13132-7.

15. Kim J, Kim H, Lee N, et al. Multifunctional uniform nanoparticles composed of a magnetite nanocrystal core and a mesoporous silica shell for magnetic resonance and fluorescence imaging and for drug delivery. Angew Chem Int Ed Engl. 2008; 47: 8438-41.

16. Chen D, Li LL, Tang FQ, et al. Facile and scalable synthesis of tailored silica "nanorattle" structures. Adv Mater. 2009; 21: 3804-7.

17. Chen Y, Chen H, Guo L, et al. Hollow/rattle-type mesoporous nanostructures by a structural difference-based selective etching strategy. ACS Nano. 2010; 4: 529-39.

18. Wong JY, Zhu L, Teo WS, et al. Revisiting the Stober method: inhomogeneity in silica shells. J. Am. Chem. Soc. 2011; 133: 11422-5.

19. Wan Y, Yu SH. Polyelectrolyte controlled large-scale synthesis of hollow silica spheres with tunable sizes and wall thicknesses. J Phys Chem C. 2008; 112: 3641-7.

20. Tan MN, Park YS. Synthesis of stable hollow silica nanospheres. J Ind Eng Chem. 2009; 15: 365-9.

21. Leng W, Chen M, Zhou S, et al. Capillary force induced formation of monodisperse polystyrene/silica organic-inorganic hybrid hollow spheres. Langmuir. 2010; 26:14271-5.

22. $\mathrm{Hu} \mathrm{J}$, Chen M, Fang $X$, et al. Fabrication and application of inorganic hollow spheres. Chem Soc Rev. 2011; 40: 5472-91.

23. Fuji M, Shin T, Watanabe H, et al. Shape-controlled hollow silica nanoparticles synthesized by an inorganic particle template method. Adv Powder Technol. 2012; 23: 562-5.

24. Wang Y, Su X, Ding P, et al. Shape-controlled synthesis of hollow silica colloids. Langmuir. 2013; 2: 11575-81.

25. Park JC, Bang JU, Lee J, et al. $\mathrm{Ni} @ S i O_{2}$ yolk-shell nanoreactor catalysts: High temperature stability and recyclability. J Mater Chem. 2010; 20: 1239-46.

26. Dahlberg KA, Schwank JW. Synthesis of $\mathrm{Ni}_{\mathrm{S}} \mathrm{SiO}_{2}$ nanotube particles in a water-in-oil microemulsion template. Chem Mater. 2012; 24: 2635-44.

27. Reddy LH, Arias JL, Nicolas J, et al. Magnetic nanoparticles: Design and characterization, toxicity and biocompatibility, pharmaceutical and biomedical applications. Chem Rev. 2012; 112: 5818-78.

28. Kim J, Lee JE, Lee J, et al. Magnetic fluorescent delivery vehicle using uniform mesoporous silica spheres embedded with monodisperse magnetic and semiconductor nanocrystals. J Am Chem Soc. 2006; 128: 688-9.

29. Roca M, Haes AJ. Silica-void-gold nanoparticles: Temporally stable surface-enhanced raman scattering substrates. J Am Chem Soc. 2008; 130: 14273-9.

30. Liz-Marzan LM, Giersig M, Mulvaney P. Homogeneous silica coating of vitreophobic colloids. Chem Commun. 1996; 0: 731-2.

31. Quyen TTB, Su WN, Chen KJ, et al. Au@SiO ${ }_{2}$ core/shell nanoparticle assemblage used for highly sensitive SERS-based determination of glucose and uric acid. J Raman Spectrosc. 2013; 44: 1671-7.

32. Lee J, Park JC, Song H. A nanoreactor framework of a Au@SiO ${ }_{2}$ yolk/shell structure for catalytic reduction of p-Nitrophenol. Adv Mater. 2008; 20: 1523-8.

33. Han L, Lv YY, Asiri AM, et al. Novel preparation and near-infrared photoluminescence of uniform core-shell silver sulfide nanoparticle@ mesoporous silica nanospheres. J Mater Chem. 2012; 22: 7274-9.

34. Lu Y, Fan H, Stump A, et al. Aerosol-assisted self-assembly of mesostructured spherical nanoparticles. Nature. 1999; 398: 223-26.

35. Suhendi A, Nandiyanto ABD, Munir MM, et al. Self-assembly of colloidal nanoparticles inside charged droplets during spray-drying in the fabrication of nanostructured particles. Langmuir. 2013; 29: 13152-61.

36. Qi J, Lai X, Wang J, et al. Multi-shelled hollow micro-/ nanostructures. Chem Soc Rev. 2015; 44: 6749-73.

37. Gratton SEA, Ropp PA, Pohlhaus PD, et al. The effect of particle design on cellular internalization pathways. Proc Natl Acad Sci USA. 2008; 105: 11613-8.

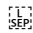

38. Liu J, Qiao SZ, Budi Hartono S, et al. Monodisperse yolk-shell nanoparticles with a hierarchical porous structure for delivery vehicles and nanoreactors. Angew Chem Int Ed. 2010; 49: 4981-5.
39. Lammers $T$, Kiessling F, Hennink WE, et al. Nanotheranostics and image-guided drug delivery: Current concepts and future directions. Mol Pharm. 2010; 7: 1899-912.

40. Turner CT, McInnes SJP, Voelcker $\mathrm{NH}$, et al. Therapeutic potential of inorganic nanoparticles for the delivery of monoclonal antibodies. J Nanomater. 2015;2015: 11 pages.

41. Maeda H. The enhanced permeability and retention (EPR) effect in tumor vasculature: the key role of tumor-selective macromolecular drug targeting. Adv Enzyme Regul. 2001; 41:189-207.

42. Porta F, Lamers GE, Morrhayim J, et al. Folic acid-modified mesoporous silica nanoparticles for cellular and nuclear targeted drug delivery. Adv Healthcare Mater. 2013; 2: 281-6.

43. Cheng K, Blumen SR, MacPherson MB, et al. Enhanced uptake of porous silica microparticles by bifunctional surface modification with a targeting antibody and a biocompatible polymer. ACS Appl Mater Interfaces. 2010; 2: 2489-95.

44. Pan L, Liu J, He Q, et al. Overcoming multidrug resistance of cancer cells by direct intranuclear drug delivery using TAT-conjugated mesoporous silica nanoparticles. Biomaterials. 2013; 34: 2719-30.

45. Li ZY, Hu JJ, Xu Q, et al. A redox-responsive drug delivery system based on RGD containing peptide-capped mesoporous silica nanoparticles. J Mater Chem. B. 2015; 3: 39-44

46. Chakravarty R, Goel S, Hong H, et al. Hollow mesoporous silica nanoparticles for tumor vasculature targeting and PET image-guided drug delivery. Nanomedicine (Lond.). 2015; 10: 1233-46.

47. Li LL, Yin $\mathrm{Q}$, Cheng JJ, et al. Polyvalent mesoporous silica nanoparticle-aptamer bioconjugates target breast cancer cells. Adv Healthcare Mater. 2012; 1: 567-72.

48. Wang $\mathrm{Z}$, Tian $\mathrm{Y}$, Zhang $\mathrm{H}$, et al. Using hyaluronic acid-functionalized ph stimuli- responsive mesoporous silica nanoparticles for targeted delivery to cD44-overexpressing cancer cells. Int J Nanomedicine. 2016; 11: 6485-97.

49. Brevet D, Gary-Bobo M, Raehm L, et al. Mannose-targeted mesoporous silica nanoparticles for photodynamic therapy. Chem Commun (Camb.). 2009; 12: 1475-7.

50. Stubbs M, McSheehy PMJ, Griffiths JR, et al. Causes and consequences of tumour acidity and implications for treatment. Mol Med Today. 2000; 6:15-9.

51. Sun JT, Hong CY, Pan CY. Fabrication of PDEAEMA-coated mesoporous silica nanoparticles and pH-responsive controlled release. J Phys Chem C 2010; 114: 12481-6.

52. Zheng J, Tian $\mathrm{X}$, Sun $\mathrm{Y}$, et al. pH-sensitive poly(glutamic acid) grafted mesoporous silica nanoparticles for drug delivery. Int J Pharm. 2013; 450: 296-303.

53. Chen $\mathrm{Y}$, Yang $\mathrm{W}$, Chang B, et al. in vivo distribution and antitumor activity of doxorubicin-loaded N-isopropylacrylamide-co-methacrylic acid coated mesoporous silica nanoparticles and safety evaluation. Eur J Pharm Biopharm. 2013; 85: 406-12

54. Li Z, Barnes JC, Bosoy A, et al. Mesoporous silica nanoparticles in biomedical applications. Chem Soc Rev. 2012; 41: 2590-605.

55. Meng $\mathrm{H}$, Xue $\mathrm{M}, \mathrm{Xia} \mathrm{T}$, et al. Autonomous in vitro anticancer drug release from mesoporous silica nanoparticles by $\mathrm{pH}$-sensitive nanovalves. J Am Chem Soc. 2010; 132: 12690-7

56. Liu R, Zhang $\mathrm{Y}$, Zhao $\mathrm{X}$, et al. $\mathrm{pH}$-responsive nanogated ensemble based on gold-capped mesoporous silica through an acid-labile acetal linker. J Am Chem Soc. 2010; 132: 1500-1.

57. Zheng $\mathrm{Q}, \mathrm{Hao} \mathrm{Y}, \mathrm{Ye} \mathrm{P}$, et al. A pH-responsive controlled release system using layered double hydroxide (LDH)-capped mesoporous silica nanoparticles. J Mater Chem B. 2013; 1 : 1644-8.

58. Muhammad F, Guo M, Qi W, et al. pH-Triggered controlled drug release from mesoporous silica nanoparticles via intracelluar dissolution of $\mathrm{ZnO}$ nanolids. J Am Chem Soc. 2011; 133: 8778-81.

59. Vasani RB, McInnes SJ, Cole MA, et al. Stimulus-responsiveness and drug release from porous silicon films ATRP-grafted with poly(N-isopropylacrylamide). Langmuir. 2011; 27: 7843-53.

60. Hu TJ, Gao J, Wu C. Swelling and shrinking of poly(N Isopropylacrylamide) chains adsorbed on the surface of polystyrene nanoparticles. J Macromol Sci Phys B. 2000; 39: 407-14.

61. Fu Q, Rao GR, Ista LK, et al. Control of molecular transport through stimuli-responsive ordered mesoporous materials. Adv Mater. 2003; 15: 1262-6.

62. Rao GV, Lopez GP. Encapsulation of poly(N-Isopropyl Acrylamide) in silica: A stimuli-responsive porous hybrid material that incorporates molecular nano-valves. Adv Mater. 2000; 12: 1692-5.

63. Liu J, Detrembleur C, Pauw-Gillet MD, Mornet S, et al. Heat-triggered drug release systems based on mesoporous silica nanoparticles filled with a maghemite core and phase-change molecules as gatekeepers. Mater Chem B. 2014; 2 : 59-70

64. Saint-Cricq P, Deshayes S, Zink JI et al. Magnetic field activated drug delivery using thermodegradable azo-functionalised PEG-coated core-shell mesoporous silica nanoparticles. Nanoscale. 2015; 7: 13168-72.

65. Karesoja M, McKee J, Karjalainen E, et al. Mesoporous silica particles grafted with poly(ethyleneoxide-block-N-vinylcaprolactam). J Polym Sci Pol Chem. 2013; 51: 5012-20.

66. Wu X, Wang $\mathrm{Z}$, Zhu $\mathrm{D}$, et al. $\mathrm{pH}$ and thermo dual-stimuli-responsive drug carrier based on mesoporous silica nanoparticles encapsulated in a copolymer-lipid bilayer. ACS Appl Mater Interfaces. 2013; 5: 10895-903. 
67. Mal NK, Fujiwara M, Tanaka Y. Photocontrolled reversible release of guest molecules from coumarin-modified mesoporous silica. Nature. 2003; 421: 350-3.

68. Tang $\mathrm{Y}, \mathrm{Hu} \mathrm{H}$, Zhang MG, et al. An aptamer-targeting photoresponsive drug delivery system using "off-on" graphene oxide wrapped mesoporous silica nanoparticles. Nanoscale. 2015; 7: 6304-10.

69. Liu J, Bu W, Pan $\mathrm{L}$, et al. NIR-triggered anticancer drug delivery by upconverting nanoparticles with integrated azobenzene-modified mesoporous silica. Angew Chem Int Ed. 2013; 52: 4375-9.

70. Zhu $\mathrm{CL}, \mathrm{Lu} \mathrm{CH}$, Song $\mathrm{XY}$, et al. Bioresponsive controlled release using mesoporous silica nanoparticles capped with aptamer-based molecular gate. J Am Chem Soc. 2011; 133: 1278-81.

71. Lai J, Shah BP, Zhang Y, et al. Real-time monitoring of ATP-responsive drug release using mesoporous-silica-coated multicolor upconversion nanoparticles. ACS Nano. 2015; 9: 5234-45.

72. Kuang WL, Zhou Q, Shen LF. Outcomes and prognostic factors of conformal radiotherapy versus intensity-modulated radiotherapy for nasopharyngeal carcinoma. Clin Transl Oncol. 2012; 14: 783-90. ișe?

73. Soares DCF, Andrada AS, Ramaldes GA. Silica nanoparticles containing gadolinium complex as potential alternative to anticancer radiotherapy. Particulate Science and Technology. 2015; 33: 331-8.

74. Ma $\mathrm{M}$, Huang $\mathrm{Y}$, Chen $\mathrm{H}$, et al. $\mathrm{Bi}_{2} \mathrm{~S}_{3}$-embedded mesoporous silica nanoparticles for efficient drug delivery and interstitial radiotherapy sensitization. Biomaterials. 2015; 37: 447-55.

75. Mumper RJ, Ryo UY, Jay M. Neutron-activated holmium-166-poly (L-lactic acid) microspheres: A potential agent for the internal radiation therapy of hepatic tumors. J. Nucl. Med. 1991; 32: 2139-43.

76. Di Pasqua AJ, Yuan $\mathrm{H}$, Chung $\mathrm{Y}$, et al. Neutron-activatable holmium-containing mesoporous silica nanoparticles as a potential radionuclide therapeutic agent for ovarian cancer. J Nucl Med. 2013; 54: 111-6.

77. Tishler RB, Geard CR, Hall EJ, et al. Taxol sensitizes human astrocytoma cells to radiation. Cancer research. 1992; 52: 3495-7.

78. Peer D, Karp JM, Hong S, et al. Nanocarriers as an emerging platform for cancer therapy. Nat Nanotechnol. 2007; 2: 751-60.

79. Ferrari M. Cancer nanotechnology: Opportunities and challenges. Nat Rev Cancer. 2005; 5: 161-71.

80. Kumar R, Belz J, Markovic S, et al. Nanoparticles based brachytherapy spacers for delivery of localized combined chemo-radiation therapy. Int J Radiat Oncol Biol Phys. 2015; 91: 393-400.

81. Ito A, Shinkai $\mathrm{M}$, Honda $\mathrm{H}$ et al. Medical application of functionalized magnetic nanoparticles. J Biosci Bioeng. 2005; 100: 1-11.

82. Sivakumar B, Aswathy RG, Nagaoka Y, et al. Aptamer conjugated theragnostic multifunctional magnetic nanoparticles as a nanoplatform for pancreatic cancer therapy. RSC Advances. 2013; 3: 20579-98.

83. Martín-Saavedra FM, Ruíz-Hernández E, Boré A, et al. Magnetic mesoporous silica spheres for hyperthermia therapy. Acta Biomaterialia. 2010; 6: 4522-31.

84. Yu X, Zhu Y. Preparation of magnetic mesoporous silica nanoparticles as a multifunctional platform for potential drug delivery and hyperthermia. Sci Technol Adv Mater. 2016; 17: 229-38.

85. Zhu Y, Tao C. DNA-capped $\mathrm{Fe}_{3} \mathrm{O}_{4} / \mathrm{SiO}_{2}$ magnetic mesoporous silica nanoparticles for potential controlled drug release and hyperthermia. RSC Advances. 2015; 5: 22365-72.

86. Ruiz-Hernández E, Baeza A, Vallet-Regí M. Smart drug delivery through DNA/magnetic nanoparticle gates. ACS Nano. 2011; 5: 1259-66.

87. Baeza A, Guisasola E, Ruiz-Hernández E et al. Magnetically triggered multidrug release by hybrid mesoporous silica nanoparticles. Chem Mat. 2012; 24: 517-24.

88. Detty MR, Gibson SL, Wagner SI. Current clinical and preclinical photosensitizers for use in photodynamic therapy. J Med Chem 2004; 47: 3897-915.

89. Zeng L, Pan $Y$, Zou R, et al. 808 nm-excited upconversion nanoprobes with low heating effect for targeted magnetic resonance imaging and high-efficacy photodynamic therapy in HER2-overexpressed breast cancer. Biomaterials. 2016; 103: 116-27.

90. Kamkaew A, Cheng L, Goel S, et al. Cerenkov radiation induced photodynamic therapy using chlorin e6-loaded hollow mesoporous silica nanoparticles. ACS Appl Mater Interfaces. 2016; 8: 26630-7.

91. Yao X, Che X, He C, et al. Dual pH-responsive mesoporous silica nanoparticles for efficient combination of chemotherapy and photodynamic therapy. J Mater Chem B. 2015; 3:4707-14.

92. Xu F, Ding L, Tao W, et al. Mesoporous-silica-coated upconversion nanoparticles loaded with vitamin B12 for near-infrared-light mediated photodynamic therapy. Materials Letters. 2016; 167: 205-8.

93. Wang H, Zhu X, Han R, et al. Near-infrared light activated photodynamic therapy of THP-1 macrophages based on core-shell structured upconversion nanoparticles. Microporous Mesoporous Mater. 2017; 239: 78-85.

94. Shanmugam V, Selvakumar S, Yeh CS. Near-infrared light-responsive nanomaterials in cancer therapeutics. Chem Soc Rev. 2014; 43: 6254-87.

95. Orecchioni M, Cabizza R, Bianco A, et al. Graphene as cancer theranostic tool: progress and future challenges. Theranostics. 2015; 5:710-23.

96. Hirsch LR, Stafford RJ, Bankson JA, et al. Nanoshell-mediated near-infrared thermal therapy of tumors under magnetic resonance guidance. Proc Natl Acad Sci USA. 2003; 100: 13549-54. SE:P:

97. Tong L, Zhao Y, Huff TB, et al. Gold nanorods mediate tumor cell death by compromising membrane integrity. Adv Mater. 2007; 19: 3136-41.
98. Peng JJ, Zhao LZ, Zhu XJ, et al. Hollow silica nanoparticles loaded with hydrophobic phthalocyanine for near-infrared photodynamic and photothermal combination therapy. Biomaterials. 2013; 34: 7905-12.

99. De Smedt SC, Demeester J, Hennink WE. Cationic polymer based gene delivery systems. Pharm. Res. 2000; 17: 113-26.

100. Panyam J, Labhasetwar V. Biodegradable nanoparticles for drug and gene delivery to cells and tissue. Adv Drug Delivery Rev. 2003; 55: 329-47.

101. PØrez-Tom $\mu$ s. Multidrug resistance: Retrospect and prospects in anti-cancer drug treatment. Curr Med Chem. 2006; 13: 1859-76.

102. Oh YK, Park TG. siRNA delivery systems for cancer treatment. Adv. Drug Delivery Rev. 2009; 61: 850-62.

103. Pakunlu RI, Wang Y, Tsao W, et al. Enhancement of the efficacy of chemotherapy for lung cancer by simultaneous suppression of multidrug resistance and antiapoptotic cellular defense. Cancer Res. 2004; 64: 6214-24.s:

104. Meng H, Mai WX, Zhang H, et al. Co-delivery of an optimal drug/siRNA combination using mesoporous silica nanoparticle to overcome drug resistance in breast cancer in vitro and in vivo. ACS Nano. 2013; 7: 994-1005. is:

105. Ma X, Zhao Y, Ng KW, et al. Integrated hollow mesoporous silica nanoparticles for target drug/siRNA co-delivery. Chem Eur J. 2013; 19: 15593-603.

106. Wu M, Meng Q, Chen Y, et al. Large pore-sized hollow mesoporous organosilica for redox-responsive gene delivery and synergistic cancer chemotherapy. Adv. Mater. 2016; 28: 1963-9.

107. Kim MH, Na HK, Kim YK, et al. Facile synthesis of monodispersed mesoporous silica nanoparticles with ultra large pores and their application in gene delivery. ACS Nano. 2011; 5: 3568-76.

108. SB Hartono, NT Phuoc, M Yu, et al. Functionalized large pore mesoporous silica nanoparticles for gene delivery featuring controlled release and co-delivery. J Mater Chem B. 2014; 2: 718-26.

109. Möller K, Müller K, Engelke H, et al. Highly efficient siRNA delivery from core-shell mesoporous silica nanoparticles with multifunctional polymer caps. Nanoscale. 2016; 8: 4007-19.

110. Zhao N, Lin X, Zhang Q, et al. Redox-triggered gatekeeper-enveloped starlike hollow silica nanoparticles for intelligent delivery Systems. Small. 2015; 11: 6467-79.

111. Weiner LM, Surana R, Wang S. Monoclonal antibodies: versatile platforms for cancer immunotherapy. Nat Rev Immunol. 2010; 10: 317-27.

112. Mahoney KM, Rennert PD, Freeman GJ. Combination cancer immunotherapy and new immunomodulatory targets. Nat Rev Drug Discov. 2015; 14: 561-84. ín

113. Lizotte PH, Wen AM, Sheen MR, et al. In situ vaccination with cowpea mosaic virus nanoparticles suppresses metastatic cancer. Nat Nanotechnol. 2016; 11:

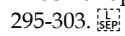

114. Makkouk A, Weiner GJ. Cancer immunotherapy and breaking immune tolerance: new approaches to an old challenge. Cancer Res. 2015; 75: 5-10.

115. Mody KT, Popat A, Mahony D, et al. Mesoporous silica nanoparticles as antigen carriers and adjuvants for vaccine delivery. Nanoscale. 2013; 5: 5167-79.

116. Mody KT, Mahony D, Zhang J, et al. Silica vesicles as nanocarriers and adjuvants for generating both antibody and T-cell mediated immune resposes to Bovine Viral Diarrhoea Virus E2 protein. Biomaterials. 2014; 35: 9972-83.

117. Heidegger S, Gößl D, Schmidt A. Immune response to functionalized mesoporous silica nanoparticles for targeted drug delivery. Nanoscale. 2016; 8: 938-48.

118. Zheng $\mathrm{H}$, Wen $\mathrm{S}$, Zhang $\mathrm{Y}$, et al. Organosilane and polyethylene glycol functionalized magnetic mesoporous silica nanoparticles as carriers for cpg immunotherapy in vitro and in vivo. PLOS ONE. 2015; 10: 0140265.

119. Lee KY, Seow E, Zhang Y, et al. Targeting CCL21-folic acid-upconversion nanoparticles conjugates to folate receptor-a expressing tumor cells in an endothelial-tumor cell bilayer model. Biomaterials. 2013; 34: 4860-71.

120. Das S, Powe AM, Baker GA, et al. Molecular fluorescence, phosphorescence, and chemiluminescence spectrometry. Anal. Chem. 2012; 84: 597-625. is is:

121. Weissleder R. A clearer vision for in vivo imaging. Nature Biotechnology. 2001; 19: 316-7.

122. Aswathy RG, Yoshida $Y$, Maekawa $T$, et al. Near-infrared quantum dots for deep tissue imaging. Anal Bioanal Chem. 2010; 397:1417-35.

123. Wang YG, Kim H, Mun S, et al. Indocyanine green-loaded perfluorocarbon nanoemulsions for bimodal 19F-magnetic resonance/nearinfrared fluorescence imaging and subsequent phototherapy. Quantitative Imaging in Medicine and Surgery. 2013; 3:132-40.

124. Lee $\mathrm{CH}$, Cheng $\mathrm{SH}$, Wang $\mathrm{YJ}$, et al. Near-Infrared mesoporous silica nanoparticles for optical imaging: Characterization and in vivo biodistribution. Adv Funct Mater. 2009; 19: 215-22.

125. Zeng C, Shang W, Wang K, et al. Intraoperative identification of liver cancer microfoci using a targeted near-infrared fluorescent probe for imaging-guided surgery. Scientific Reports. 2016; 6: 21959.

126. Luo T, Huang P, Gao G, et al. Mesoporous silica-coated gold nanorods with embedded indocyanine green for dual mode X-ray CT and NIR fluorescence imaging. Opt Express. 2011; 19: 17030-9.

127. Xie M, Shi H, Li Z, et al. A multifunctional mesoporous silica nanocomposite for targeted delivery, controlled release of doxorubicin and bioimaging. Colloids Surf. B. 2013; 110: 138-47.

128. Xue S, Wang Y, Wang M, et al. Iodinated oil-loaded, fluorescent mesoporous silica-coated iron oxide nanoparticles for magnetic resonance 
imaging/computed tomography/fluorescence trimodal imaging. Int J Nanomedicine. 2014; 9:2527-38.

129. Chen F, Hong H, Shi SX, et al. Engineering of hollow mesoporous silica nanoparticles for remarkably enhanced tumor active targeting efficacy. Sci Rep. 2014; 4: 5080

130. Song JT, Yang XQ, Zhang XS, et al. Facile synthesis of gold nanospheres modified by positively charged mesoporous silica, loaded with near-infrared fluorescent dye, for in vivo x-ray computed tomography and fluorescence dual mode imaging. ACS Applied Materials \& Interfaces. 2015; 7: 17287-97.

131. W Fan, B Shen, W Bu, et al. A smart upconversion-based mesoporous silica nanotheranostic system for synergetic chemo-/radio-/photodynamic therapy and simultaneous MR/UCL imaging. Biomaterials. 2014; 35: 8992-9002.

132. Su X, Zhao F, Wang Y, et al. CuS as a gatekeeper of mesoporous upconversion nanoparticles-based drug controlled release system for tumor-targeted multimodal imaging and synergetic chemo-thermotherapy. Nanomedicine: Nanotechnology, Biology and Medicine. 2017; 13: 1761-72.

133. Zhao $\mathrm{P}$, Zhu $\mathrm{YH}$, Yang $\mathrm{XL}$, et al. Multifunctional $\mathrm{MnO}_{2}$ nanosheet-modified $\mathrm{Fe}_{3} \mathrm{O}_{4} @ \mathrm{SiO}_{2} / \mathrm{NaYF}_{4}: \mathrm{Yb}$, Er nanocomposites as novel drug carriers. Dalton Trans. 2014; 43: 451-7.

134. Zhou L, Zheng X, Gu Z, et al. Mesoporous NaYbF4@NaGdF4 core-shell up-conversion nanoparticles for targeted drug delivery and multimodal imaging. Biomaterials. 2014; 35: 7666-78.

135. Huang X, Zhang F, Lee S, et al. Long-term multimodal imaging of tumor draining sentinel lymph nodes using mesoporous silica-based nanoprobes. Biomaterials. 2012; 33:4370-8.

136. Hayashi K, Nakamura M, Ishimura K. Near-infrared fluorescent silica-coated gold nanoparticle clusters for x-ray computed tomography/optical dual modal imaging of the lymphatic system. Adv healthcare mater. 2012; 2: 756-63.

137. Na HB, Hyeon T. Nanostructured T1 MRI contrast agents. J Mater Chem. 2009; 19: $6267-73$.

138. Spernyak JA, WhiteIII WH, Ethirajan M, et al. Hexylether derivative of pyropheophorbide-a (HPPH) on conjugating with 3Gadolinium(III) aminobenzyldiethylenetriaminepentaacetic acid shows potential for in vivo tumor imaging (MR, fluorescence) and photodynamic therapy. Bioconjugate Chem. 2010; 21: 828-35.

139. Hurley KR, Lin YS, Zhang J, et al. Effects of mesoporous silica coating and postsynthetic treatment on the transverse relaxivity of iron oxide nanoparticles. Chem Mater. 2013; 25:1968-78.

140. Yang H, Zhuang Y, Hu H, et al. Silica-coated manganese oxide nanoparticles as a platform for targeted magnetic resonance and fluorescence imaging of cancer cells. Adv Funct Mater. 2010; 20: 1733-41.

141. Vivero-Escoto JL, Taylor-Pashow KML, Huxford RC, et al. Multifunctional mesoporous silica nanospheres with cleavable Gd(III) chelates as MRI contrast agents: synthesis, characterization, target-specificity, and renal clearance. Small. 2011; 7: 3519-28

142. Wu M, Meng Q, Chen $\mathrm{Y}$, et al. Ultrasmall confined iron oxide nanoparticle MSNs as a pH-responsive theranostic platform. Adv Funct Mater. 2014; 24: 4273-83.

143. Lee JE, Lee N, Kim T, et al et al. T. Multifunctional mesoporous silica nanocomposite nanoparticles for theranostic applications. Acc Chem Res. 2011; 44: 893-902.

144. Jiang Y, Liu S, Zhang Y, et al. Magnetic mesoporous nanospheres anchored with LyP-1 as an efficient pancreatic cancer probe. Biomaterials. 2017; 115:9-18.

145. Yang G, Gong H, Liu T, et al. Two-dimensional magnetic $\mathrm{WS}_{2} @ \mathrm{Fe}_{3} \mathrm{O}_{4}$ nanocomposite with mesoporous silica coating for drug delivery and imaging-guided therapy of cancer. Biomaterials. 2015; 60: 62-71.

146. Manus LM, Mastarone DJ, Waters EA, et al. Gd(III)-nanodiamond conjugates for MRI contrast enhancement. Nano Lett 2010; 10: 484-9.

147. Yang D, Yang G, Gai S, et al. Imaging-guided and light-triggered chemo-/photodynamic/ photothermal therapy based on Gd (III) chelated mesoporous silica hybrid spheres. ACS Biomater Sci Eng. 2016; 2: 2058-71.

148. Chen Y, Ai K, Liu J, et al. Multifunctional envelope-type mesoporous silica nanoparticles for $\mathrm{pH}$-responsive drug delivery and magnetic resonance imaging. Biomaterials. 2015; 60: 111-20.

149. Shao YZ, Liu LZ, Song SQ,et al. A novel one-step synthesis of $\mathrm{Gd}^{3+}$-incorporated mesoporous $\mathrm{SiO}_{2}$ nanoparticles for use as an efficient MRI contrast agent. Contrast Media Mol Imaging. 2011; 6: 110-8.

150. Pan D, Caruthers SD, Senpan A, et al. Revisiting an old friend: manganese-based MRI contrast agents. Wiley Interdiscip Rev Nanomed. Nanobiotechnol. 2011; 3:162-73

151. Niu D, Luo X, Li Y, et al. Manganese loaded dual mesoporous silica spheres for efficient T1- and T2-Weighted dual mode magnetic resonance imaging. ACS Appl Mater Interfaces. 2013; 5: 9942-8.

152. Li X, Zhao W, Liu X, et al. Mesoporous manganese silicate coated silica nanoparticles as multi-stimuli-responsive T1-MRI contrast agents and drug delivery carriers. Acta Biomaterialia. 2016; 30: 378-87.

153. Hu H, Dai A, Sun J, et al. Aptamer-conjugated $\mathrm{Mn}_{3} \mathrm{O}_{4} @ \mathrm{SiO}_{2}$ core-shell nanoprobes for targeted magnetic resonance imaging. Nanoscale. 2013; 5: 10447-54.

154. Phelps ME. Positron emission tomography provides molecular imaging of biological processes. Proc Natl Acad Sci USA. 2000; 97: 9226-33.

155. Ametamey SM, Honer M, Schubiger PA. Molecular imaging with PET. Chem Rev. 2008; 108: 1501-16.s:

156. Gambhir SS. Molecular imaging of cancer with positron emission tomography. Nat Rev. 2002; 2: 683-93.
157. Chen F, Hong $\mathrm{H}$, Zhang $\mathrm{Y}$, et al. in vivo tumor targeting and image-guided drug delivery with antibody-conjugated, radiolabeled mesoporous silica nanoparticles. ACS Nano. 2013; 7: 9027-39. iscep:

158. Lee SB, Kim HL, Jeong HJ, et al. Mesoporous silica nanoparticle pretargeting for PET imaging based on a rapid bioorthogonal reaction in a living body. Angew Chem Int Ed Engl. 2013; 52:10549-52.

159. Chen F, Nayak TR, Goel S, et al. in vivo tumor vasculature targeted pet/nirf imaging with TRC105 (Fab) conjugated, dual-labeled mesoporous silica nanoparticles. Mol. Pharmaceutics. 2014; 11: 4007-14.

160. Chen F, Hong $\mathrm{H}$, Goel S, et al. in vivo tumor vasculature targeting of CuS@MSN based theranostic nanomedicine. ACS Nano. 2015; 9: 3926-34.

161. Miller L, Winter G, Baur B, et al. Synthesis, characterization, and biodistribution of multiple $89 \mathrm{Zr}$-labeled pore-expanded mesoporous silica nanoparticles for PET. Nanoscale. 2014; 6: 4928-35.

162. Popovtzer R, Agrawal A, Kotov NA, et al. Targeted gold nanoparticles enable molecular CT imaging of cancer. Nano Lett. 2008; 8: 4593-6.

163. Oh MH, Lee N, Kim H, et al. Large-scale synthesis of bioinert tantalum oxide nanoparticles for $\mathrm{x}$-ray computed tomography imaging and bimodal image-guided sentinel lymph node mapping. J Am Chem Soc. 2011; 133: 5508-15.

164. Liu Y, Ai K, Liu J, et al. A high-performance ytterbium-based nanoparticulate contrast agent for in vivo X-ray computed tomography imaging. Angew Chem Int Ed. 2012; 51: 1437-42.

165. Ai K, Liu Y, Liu J, et al. Large-scale synthesis of $\mathrm{Bi}_{2} \mathrm{~S}_{3}$ nanodots as a contrast agent for in vivo X-ray computed tomography imaging. Adv Mater. 2011; 23: 4886-91.

166. Ahn S, Jung SY, Seo E, et al. Gold nanoparticle-incorporated human red blood cells (RBCs) for X-ray dynamic imaging. Biomater. 2011; 32: 7191-9.

167. Peng C, Zheng L, Chen $Q$, et al. PEGylated dendrimer-entrapped gold nanoparticles for in vivo blood pool and tumor imaging by computed tomography. Biomater. 2012; 33: 1107-19.

168. Schulz M, Ma-Hock L, Brill S, et al. Investigation on the genotoxicity of different sizes of gold nanoparticles administered to the lungs of rats. Mutat Res. 2012; 745: 51-7.

169. Park MVDZ, Neigh AM, Vermeulen JP, et al. The effect of particle size on the cytotoxicity, inflammation, developmental toxicity and genotoxicity of silver nanoparticles. Biomater. 2011; 32: 9810-7.

170. Shen S, Tang H, Zhang X, et al. Targeting mesoporous silica-encapsulated gold nanorods for chemo-photothermal therapy with near-infrared radiation. Biomater. 2013; 34: 3150-8.

171. Wang TT, Chai F, Wang CG, et al. Fluorescent hollow/rattle-type mesoporous $\mathrm{Au} @ \mathrm{SiO}_{2}$ nanocapsules for drug delivery and fluorescence imaging of cancer cells. J Colloid Inter Sci. 2011; 358: 109-15.

172. Li X, Xing L, Zheng K, et al. Formation of gold nanostar-coated hollow mesoporous silica for tumor multimodality imaging and photothermal therapy. ACS Appl Mater Interfaces. 2017; 9: 5817-27.

173. Zeng C, Shang W, Liang $X$, et al. Cancer diagnosis and imaging-guided photothermal therapy using a dual-modality nanoparticle. ACS Appl Mater Interfaces. 2016; 8: 29232-41.

174. Son S, Min HS, You DG, et al. Echogenic nanoparticles for ultrasound technologies: Evolution from diagnostic imaging modality to multimodal theranostic agent. Nano Today. 2014; 9: 525-40.

175. Yin TH, Wang $P$, Zheng RQ, et al. Nanobubbles for enhanced ultrasound imaging of tumors. Int J Nanomed. 2012; 7: 895-904.

176. Liu J, Levine AL, Mattoon JS, et al. Nanoparticles as image enhancing agents for ultrasonography. Phys Med Biol. 2006; 51: 2179-89.

177. Liu J, Li J, Rosol TJ, et al. Biodegradable nanoparticles for targeted ultrasound imaging of breast cancer cells in vitro. Phys Med Biol. 2007; 52: 4739-47.

178. Chen F, Ma M, Wang J, et al. Exosome-like silica nanoparticles: a novel ultrasound contrast agent for stem cell imaging. Nanoscale. 2017; 9: 402-11.

179. Yildirim A, Chattaraj R, Blum NT, et al. Understanding acoustic cavitation initiation by porous nanoparticles: Toward nanoscale agents for ultrasound imaging and therapy. Chem Mater. 2016; 28: 5962-72.

180. Yildirim A, Chattaraj R, Blum NT, et al. Stable encapsulation of air in mesoporous silica nanoparticles: Fluorocarbon-free nanoscale ultrasound contrast agents. Adv Healthc Mater. 2016; 5: 1290-8.

181. Qian X, Wang W, Kong W, et al. Organic-inorganic hybrid hollow mesoporous organosilica nanoparticles for efficient ultrasound-based imaging and controlled drug release. J Nanomater. 2014; 2014: 972475, 8 pages.

182. Jin Q, Lin CY, Kang ST, et al. Superhydrophobic silica nanoparticles as ultrasound contrast agents. Ultrasonics Sonochemistry. 2017; 36: 262-9.

183. Martinez HP, Kono Y, Blair SL, et al. Hard shell gas-filled contrast enhancement particles for colour Doppler ultrasound imaging of tumors. Med Chem Comm. 2010; 1:266-70.

184. Liberman A, Wu Z, Barback CV, et al. Color doppler ultrasound and gamma imaging of intratumorally injected $500 \mathrm{~nm}$ iron silica nanoshells. ACS Nano. 2013; 7: 6367-77.

185. Zhang K, Chen H, Guo X et al Double-scattering/reflection in a single nanoparticle for intensified ultrasound imaging. Sci Rep.2015; 5:8766.

186. Le Ru EC, Blackie E, Meyer M, et al. Surface enhanced Raman scattering enhancement factors: A comprehensive study. J Phys Chem C. 2007; 111:13794-803.

187. Pal S, Harmsen S, Oseledchyk A, et al. MUC1 aptamer targeted SERS nanoprobes. Adv Funct Mater. 2017; 27: 1606632. 
188. Harmsen S, Huang R, Wall MA, et al. Surface-enhanced resonance Raman scattering nanostars for high-precision cancer imaging. Sci Transl Med. 2015; 7:271ra7.

189. Wall MA, Shaffer TM, Harmsen S, et al. Chelator-free radiolabeling of SERRS nanoparticles for whole-body PET and intraoperative Raman imaging. Theranostics. 2017; 7: 3068-77.

190. Neuschmelting V, Harmsen S, Beziere N, et al. Dual-modality surface-enhanced resonance Raman scattering and multispectral optoacoustic tomography nanoparticle approach for brain tumor delineation. Small. 2018; 14: 1800740

191. Park JH, Gu L, von Maltzahn G, et al. Biodegradable luminescent porous silicon nanoparticles for in vivo applications. Nat. Mater. 2009; 8: 331-6.

192. Durfee PN, Lin YS, Dunphy DR, et al. Mesoporous silica nanoparticle-supported lipid bilayers (protocells) for active targeting and delivery to individual leukemia cells. ACS Nano. 2016; 10: 8325-45.

193. Cheng W, Nie J, Xu L, et al. A pH-sensitive delivery vehicle based on folic acid-conjugated polydopamine-modified mesoporous silica nanoparticles for targeted cancer therapy. ACS Appl. Mater. Interfaces. 2017; 9: 18462-73.

194. Zhou S, Wu D, Yin $\mathrm{X}$, et al. Intracellular pH-responsive and rituximab-conjugated mesoporous silica nanoparticles for targeted drug delivery to lymphoma B cells. J Exp. Clin. Cancer Res. 2017; 36: 24

195. Cui J, De Rose R, Alt K, et al. Engineering Poly(ethylene glycol) particles for improved biodistribution. ACS Nano. 2015; 9:1571-80.

196. Rascol E, Daurat M, Da Silva, et al. Biological fate of $\mathrm{Fe}_{3} \mathrm{O}_{4}$ core-shell mesoporous silica nanoparticles depending on particle surface chemistry. Nanomaterials. 2017; 7: 162.

197. Kostiv U, Rajsiglová L, Luptáková D, et al. Biodistribution of upconversion/magnetic silica-coated NaGdF4:Yb3+/Er3+ nanoparticles in mouse models. RSC Adv. 2017; 7: 45997-6006. 